WellBeing International

WBI Studies Repository

2007

\title{
The Human/Animal Interface: Emergence and Resurgence of Zoonotic Infectious Diseases
}

Michael Greger

The Humane Society of the United States

Follow this and additional works at: https://www.wellbeingintlstudiesrepository.org/acwp_tzd

Part of the Animal Studies Commons, Other Animal Sciences Commons, and the Veterinary Infectious Diseases Commons

\section{Recommended Citation}

Greger, M. (2007). The human/animal interface: emergence and resurgence of zoonotic infectious diseases. Critical Reviews in Microbiology, 33(4), 243-299.

This material is brought to you for free and open access by WellBeing International. It has been accepted for inclusion by an authorized administrator of the WBI Studies Repository. For more information, please contact wbisr-info@wellbeingintl.org.

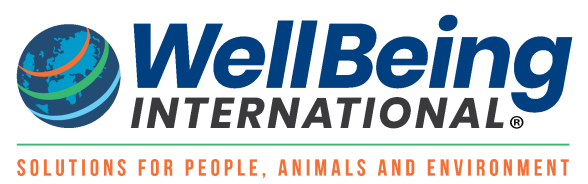




\title{
The Human/Animal Interface: Emergence and Resurgence of Zoonotic Infectious Diseases
}

\author{
Michael Greger \\ The Humane Society of the United States \\ CITATION
}

Greger, M. (2007). The human/animal interface: emergence and resurgence of zoonotic infectious diseases. Critical reviews in microbiology, 33(4), 243-299.

\section{KEYWORDS}

agriculture, Avian Influenza, Borrelia burgdorferi, Bovine Spongiform Encephalopathy, bushmeat, Campylobacter; concentrated animal feeding operations, deltaretroviruses, disease ecology, disease evolution, domestic fowl, emerging infectious diseases, Escherichia coli O157, extraintestinal pathogenic Escherichia coli, farm animals, HIV, Influenza A Virus Subtype H5N1, Listeria monocytogenes, multiple drug resistance, Nipah Virus, pandemics, pets, public health, Salmonella, SARS Virus, Simian Foamy Virus, Streptococcus suis, viral Hemorrhagic Fevers, virulence, zoonoses

\begin{abstract}
Emerging infectious diseases, most of which are considered zoonotic in origin, continue to exact a significant toll on society. The origins of major human infectious diseases are reviewed and the factors underlying disease emergence explored. Anthropogenic changes, largely in land use and agriculture, are implicated in the apparent increased frequency of emergence and re-emergence of zoonoses in recent decades. Special emphasis is placed on the pathogen with likely the greatest zoonotic potential, influenzavirus $\mathrm{A}$.
\end{abstract}

\section{INTRODUCTION}

Absent the many other impacts of emerging infectious diseases, an analysis solely of the economic ramifications indicates the costs may be substantial. Based on net changes in GDP growth forecasts, official (Fan 2003) and academic (Lee and McKibbin 2003) estimates of the economic costs of SARS in Asia exceed 10 billion USD. Extrapolating this regional GDP drop globally, according to the World Bank's calculus, an influenza pandemic could represent a loss of 800 billion USD (Brahmbhatt 2005), though the financial implications of a pandemic may surpass SARS in scope and duration (McKibbin and Sidorenko 2006).

The 1957 and 1968 influenza pandemics cost an estimated 26.8 billion USD (Lederberg et al. 1992), and highly pathogenic avian influenza (HPAl) expenditures are already in the billions (McLeod et al. 2005). The United Kingdom lost approximately 6 billion USD due to bovine spongiform encephalopathy (Davis and Lederberg 2001), and the costs associated with antimicrobial resistance continue to rise (McGowan 2001). HIVIAIDS may have cost 500 billion USD so far (Annan 2001). HIV now infects an estimated 38.6 million people, propelling AIDS to the leading cause of premature death among people 15 to 59 years of age worldwide (Merson 2006).

The economic, social, and public health burden of these diseases begs the question: from where do emerging diseases emerge? 
Most human diseases, old and new, are caused by multi-host pathogens. In a review of 1,407 species of human pathogenic organisms, $816(58 \%)$ were broadly classified as zoonotic (Woolhouse and GowtageSequeria 2005), a term coined by Virchow and defined by the World Health Organization in 1959 to describe "those diseases and infections (the agents of) which are naturally transmitted between (other) vertebrate animals and man" (Mantovani 2001).

The exact proportion of emerging human diseases that have arisen from nonhuman animals is unknown. However, according to the Woolhouse and Gowtage-Sequeria review (2005), of the 177 of 1,407 human pathogens identified as "emerging," $130(73 \%)$ were zoonotic. The landmark U.S. Institute of Medicine (IOM) report Emerging Infections concluded that "[t]he significance of zoonoses in the emergence of human infections cannot be overstated" (Lederberg et al. 1992).

\section{A. The First Epidemiological Transition: Domestic Origins}

Although there are likely "heirloom" pathogens with which we co-evolved throughout the evolutionary chain from Homo to Homo sapiens (Cockburn 1967), most modern human infectious diseases may have been unknown to our hunter and gatherer ancestors. From the Pleistocene Epoch until fewer than 11,000 years ago, the human population is thought to have existed as small, nomadic groups of a size and density that precluded the existence of short-lived, immunizing, human-specific infections, a proposal strongly put forth by Burnet (1946) and later supported by increasingly complex mathematical modeling (Dobson and Carper 1996).

Medical anthropologists have identified a series of "epidemiological transitions," major shifts in human disease, since the beginning of human evolution six million years ago. The advent of agriculture arguably triggered the first epidemiological transition, as population size and density grew to levels ripe for the introduction and maintenance of "crowd diseases." Communities planting crops settled and grew in relatively fixed locations. This likely increased their exposure to their own waste as well as to reservoirs of disease such as unintentional rodent domesticants that may have set up permanent habitats proximate to human dwellings (Armelagos et al. 2005).

Although malaria predates agricultural societies, the transition may have led to rapid speciation and spread of anthropophilic mosquito vectors (Coluzzi 1999), helping to explain the expansion of virulent Plasmodium falciparum in Africa (Joy et al. 2003). Similarly, the domestication of cats roughly coincides with the acquisition of efficient oral transmission and subsequent expansion of Toxoplasma (Su et al. 2003).

The transformation of Yersinia pseudotuberculosis, a relatively benign fecal-oral route bacillus, into Yersinia pestis, the flea-transmitted cause of bubonic plague, may have also been coincident with the rise of agriculture and subsequent food supply for rodent hosts (Achtman et al. 1999). Similar changes in rodent ecology have been identified as risk factors for the reappearance of human cases of plague in recent years (Duplantier et al. 2005).

Although controversy continues to surround the origin of many infectious diseases (Pearce-Duvet 2006), many of humanity's most serious seem to have been the result of increasingly close and frequent contact with a new array of potentially zoonotic pathogens from animals intentionally domesticated for human use (Ritvo 2004). The same quality that made certain animals particularly desirable for domestication-the herd instinct-may have also selected for species that herd or flock together in concentrations large enough to harbor epizootic pathogens capable of rapid, population-wide spread (Diamond 1997). Early humans may have suffered sporadic cases of animal-borne diseases, such as anthrax from wild sheep or tularemia ("rabbit skinner's disease") from wild rabbits (Weber and Rutala 1999), but the domestication of animals around 10,000 years ago likely provided the greatest conduit for zoonotic disease transmission (Wolfe et al. 2007).

Human measles likely arose from a rinderpest-like virus of sheep and goats (Weiss 2001). Only with the prolonged, intimate contact of domestication was this virus thought able to make the species jump (Daszak and Cunningham 2002). Smallpox may have resulted from camel domestication, with camelpox 
arising from a cowpox-like, rodent-borne ancestor (Gubser et al. 2004). Pertussis may have had an ovine or porcine source (Weiss 2001). In exchange, domestication presented the opportunity to relay to both cattle and pigs the tapeworms (Taenia) hominids likely acquired scavenging bovid carcasses (Hoberg et al. 2001).

Human influenza may have only started about 4,500 years ago with the domestication of waterfowl (Shortridge 2003b), and leprosy may have originated in water buffalo (McMichael 2001a). Phylogenetic analysis suggests human rhinovirus may have come from cattle (Rodrigo and Dopazo 1995). Before domestication, the common cold may have been common only to them.

Mycobacterium tuberculosis was thought to have derived from Mycobacterium bovis (Sreevatsan et al. 1997), but more recent evidence suggests a common ancestor (Brosch et al. 2002). This is consistent with its remarkable ability to persist for decades in host tissues, suggesting a longer period of coadaptation. Indeed, unlike pathogens such as rinderpest virus and camelpox that, today, pose very little or no human risk (Jezek 1983), tuberculosis mycobacteria continue to present a zoonotic threat to public health, particularly in enzootic areas of the developing world with high rates of HIV infection (Ayele et al. 2004). Worldwide, M. bovis TB, which is clinically indistinguishable in humans from M. tuberculosis TB, is estimated to account for $3 \%$ of all manifestations of human tuberculosis (Cosivi et al. 1998). This underscores the importance of reinforcing milk pasteurization, animal TB control programs, and airway protection for meat industry workers (Thoen et al. 2006), and, perhaps, the deintensification of captive deer farming (Wilson 2002). Incidentally zoonotic transmission may be bidirectional with molecular-typed confirmation of human pulmonary TB transmission to cattle (Ocepek et al. 2005), as well as cases of bovine infection linked to farm workers with genitourinary tuberculosis urinating on hay (Collins 2000).

All of these animals-birds, bovines, camels, sheep, and swine-were domesticated exclusively in Eurasia, which may explain the unilaterality by which indigenous populations in the Americas were devastated by diseases such as smallpox brought by the landing Europeans (Diamond 2002). Entire Amerindian civilizations may have fallen to diseases born on the farm.

Pleistocene Ice Age extinctions eliminated most of the large mammalian species in the Western hemisphere. With the disappearance of American camels and horses, for example, indigenous populations were left with only animals such as llamas and guinea pigs to domesticate, neither of which seem to carry much potential for zoonotic disease. This placed the American indigenous peoples in a position both enviable and vulnerable: Humans in the Americas had the advantage of living relatively free of animal-borne diseases, yet, in the apparent absence of prior exposure, resistance, or immunity to livestock-derived zoonoses, faced grave risk when introduced to such immunologically novel pathogens. In fact, livestock-derived zoonotic diseases contributed to the loss of as much as $90 \%$ of the native American population (Jones 2004). There were buffalo in the Americas, for example, but no domesticated buffalo, so there was presumably no opportunity for a disease like measles to arise until it was brought by Eurasians. Indeed, it is difficult to pin down a single disease that instead sailed eastbound across the Atlantic (Weiss 2003).

Additional infections that may have originated with animal agriculture continue to be discovered. Helicobacter pylori, the bacteria for which the 2005 Nobel Prize in Medicine was awarded, may have first been transmitted to humans via sheep's milk and is now thought to spread horizontally in the human population (Dore et al. 2001). Roughly half of the world's population may now be infected with $H$. pylori, making this peptic ulcer and stomach cancer-associated infection perhaps the most common chronic infection afflicting humanity (Suerbaum and Michetti 2002). The closely related Helicobacter pullorum, a recent addition to the list of zoonotic farm animal bacteria (Stanley et al. 1994), contaminates a large proportion of retail chicken meat (Atabay and Corry 1998), and may result in a human foodborne diarrheal illness (De Groote et al. 2000).

Another newly described fecal pathogen, hepatitis $E$ virus (HEV), is now recognized as a leading cause of fulminant liver failure in developing countries (Worm et al. 2002), particularly in pregnant women, with a case fatality of up to $39 \%$ found in a prospective study of women presenting in their third trimester with acute HEV hepatitis (Beniwal et al. 2003). Scientists began to suspect the zoonotic nature of this virus 
when it was found rampant in North American commercial pork operations (Yoo et al. 2001). Direct evidence of cross-species transmission was obtained in 2003 (Tei et al. 2003). Recent isolation of infectious HEV from commercial pig liver sold in U.S. grocery stores heightens the public health concern over zoonotic HEV foodborne transmission (Feagins et al. 2007). Unlike dead-end diseases like trichinosis transmitted by eating improperly cooked pork, once a disease like hepatitis $E$ crosses the species line, it may spread human-to-human via blood transfusion (Boxall et al. 2006) or the fecal-oral route, resulting in large outbreaks from contaminated water (Myint and Gibbons 2007).

\section{B. The Second Epidemiological Transition: Complacency}

The second epidemiological transition arguably began a century or so ago in countries where improved nutrition, public health measures, and medical intervention led to both a decline in infectious disease (Omran 1971) and what the U.S. Institute of Medicine report called the "era of complacency" (Davis and Lederberg 2001). In 1948, the U.S. Secretary of State pronounced that the conquest of all infectious diseases was imminent (Najera 1989). Nineteen years later, victory was declared by U.S. Surgeon General William Stewart: "The war against diseases has been won" (Morens et al. 2004).

Though premature, Dr. Stewart's declaration may have been understandable. By that time in the United States, polio and rheumatic fever had been conquered, smallpox nearly eradicated, childhood vaccinations developed, and an arsenal of more than 25,000 different "miracle drug" antibiotic preparations assembled (Selgelid 2005). A Nobel Prize-winning virologist was even seduced into the heady optimism. In the 1962 text Natural History of Infectious Disease, Frank Macfarlane Burnet cowrote: "To write about infectious disease is almost to write of something that has passed into history. . . [T]he most likely forecast about the future of infectious disease," he continued, "is that it will be very dull" (Burnet and White 1962).

\section{The Third Epidemiological Transition: Emerging Infectious Diseases}

The situation soon changed. For the first time since the U.S. federal government began tracking mortality statistics, the declining domestic trend in infectious disease mortality reversed in 1981, a shift attributed largely to AIDS deaths (Armstrong et al. 1999). We may currently be living in the third epidemiological transition, which started approximately 30 years ago, the emergence (or re-emergence) of infectious diseases (Armelagos et al. 2005) (though it can be argued that if counting intercontinental European expansion, we are currently in the midst of the fourth) (McMichael 2004).

The concept of "emerging infectious diseases" has changed from a mere curiosity in the field of medicine to an entire discipline that has been gaining prominence (Brown 2000a). In recent decades, previously unknown diseases have surfaced at a pace unheard of in the recorded annals of medicine (Smolinski et al. 2003)—-more than 30 newly identified human pathogens in 30 years, most of them newly-discovered zoonotic viruses (Woolhouse 2002).

Although many of today's doctors learned in their medical school textbooks that viruses were speciesspecific (Clough 2004), we now know interspecies navigation to be common. Creative "kingdom jumping" speculations of a plant reservoir for Ebola virus aside (Monath 1999), "novel" viruses, as obligate parasites, tend to come from other animal species almost by definition (Wain-Hobson and Meyerhans 1999).

The trend of increasing zoonotic virus emergence is expected to continue (WHO/FAO/OIE 2004), and estimates of the potential number of zoonotic diseases already run into the thousands (Beran and Steele 1994). The 2003 follow-up to the 1992 Institute of Medicine report on emerging infections suggests that without appropriate policies and actions, the future could bring a "catastrophic storm of microbial threats" (Smolinski et al. 2003).

The first surge in species-barrier breaches likely occurred with the clustering of zoonotic vectors accompanying the domestication of animals-but that was 10,000 years ago. What new changes are 
taking place at the human/animal interface that may be responsible for this resurgence of zoonotic disease in recent decades?

\section{Anthropogenic Impact}

Starting in the last quarter of the 20th century, medicine has been examining emerging infections within an increasingly ecological framework, although the concept is not new. Two centuries ago, Edward Jenner, the founder of modern vaccines, proposed that the "deviation of man from the state in which he was originally placed by nature seems to have proved to him a prolific source of diseases" (McMichael 2004). This observation evidently dates back to the 2nd century, when Plutarch argued that new classes of diseases followed profound changes in the way we live (Drexler 2002).

The distinctions raised in the disease emergence debate framed by Schrag and Wiener (1995) regarding the relative contribution of changes in host versus pathogen and ecology versus evolution may be largely artificial. As exemplified by the emergence of antibiotic-resistant bacteria, disease emergence may be viewed as an evolutionary response to largely anthropogenic environmental changes (Slingenbergh et al. 2004).

The recent rise in zoonotic disease emergence has been paralleled by an apparent increase in nonzoonotic infectious disease emergence among domesticated animals (Mahy and Brown 2000), wild animals (Daszak et al. 2000), domesticated plants (Rybicki and Pietersen 1999), and wild plants (Anderson and May 1986). Palumbi (2001) argues that humans are currently the world's greatest evolutionary force.

In 2004, a joint consultation on emerging zoonoses was convened by the World Health Organization, the Food and Agriculture Organization of the United Nations, and the World Organization for Animal Health to elucidate the major drivers of zoonotic disease emergence (WHO/FAO/OIE 2004). A common theme of primary risk factors for the emergence and spread of emerging zoonoses was the increasing demand for animal protein, associated with the expansion and intensification of animal agriculture, long-distance live animal transport, live animal markets, bushmeat consumption, and habitat destruction.

\section{HABITAT DESTRUCTION}

In 1933, wildlife ecology pioneer Aldo Leopold reportedly wrote that "[t]he real determinants of disease mortality are the environment and the population," both of which he felt were being "doctored daily, for better or for worse, by gun and axe, and by fire and plow" (Friend et al. 2001). Since Leopold penned those lines, more than half of the Earth's tropical forests have been cleared (Pimm et al. 2001). Based on satellite imaging, global tropical deforestation continues at an annual loss of up to $2-3 \%$ (Patz et al. 2004), with the net rate of tropical forest clearing increasing roughly $10 \%$ from the 1980 s to 1990 s (DeFries et al. 2002).

Grazing animals for human consumption demands an estimated 0.21 hectares per global capita, comparable with the 0.22 hectacres attributed to timber harvesting (Wackernagel et al. 2002). One hundred thousand years ago, likely all members of the human race lived in eastern Africa (McMichael 2001b). The world's population has grown from fewer than 100 million people 3,000 years ago to approximately 1 billion at the turn of the 20th century, more than 6 billion by the end of the century, with 2050 projections approaching 10 billion (Tilman et al. 2001). Two-thirds of this population increase occurred within the last 50 years (De Souza et al. 2003), leading to major ecological changes and wildlife habitat reduction implicated in zoonotic disease emergence (Chomel et al. 2007).

\section{A. Nipah Virus}

In 1998, a large outbreak of severe, febrile encephalitis occurred among Malaysians exposed to intensively-farmed pigs that were sickened by fruit bats displaced by slash-and-burn deforestation. The disease, named after Sungai Nipah New Village from which the virus was first isolated, was spread by infected pigs transported around the country. The emergence of Nipah virus demonstrates the interplay 
between multiple ecological risk factors cited in the WHO/FAO/OIE report: habitat destruction, intensive animal agriculture, and long-distance animal transport.

Globally, the rate of tropical deforestation is highest in Asia (Mayaux et al. 2005). In 1997 and 1998 more than 10 million acres of virgin forest burned in Borneo and Sumatra, set ablaze by humans but exacerbated by a severe El Nĩno Southern Oscillation event drought. The haze and habitat loss are thought to have caused a mass exodus of "flying fox" fruit bats (Pteropus) searching for food, resulting in an unprecedented encroachment on cultivated fruit trees (Chua et al. 2002a). Planting orchards next to pig farms allows for efficient access to swine manure for fertilizer, but may also result in porcine exposure to flying fox saliva from partially eaten fruit or urine, from both of which the novel paramyxovirus was isolated (Chua et al. 2002b).

Though remaining harmless to fruit bats, the Nipah virus acquired the capacity to cause a severe neurological and respiratory syndrome in young pigs characterized by a loud, nonproductive, "barking" cough (Mohd et al. 2000). Displaying an unusual host promiscuity, the virus caused respiratory distress in other animals proximate to the pig farm-goats, sheep, dogs, cats, and horses (Uppal 2000). The subsequent trucking of infected pigs to five states inMalaysia and into Singapore resulted in 229 human cases, nearly half of which (48\%) ended in fatality (CDC 1999). A cull of more than 1 million pigs in affected areas effectively ended the outbreak (Lam 2003).

Importantly, previous sporadic deaths of pig farm workers exposed to encephalitic pigs had been reported elsewhere in Malaysia the year earlier (Bunning et al. 2000). It may be no coincidence that the index farm triggering the large outbreak was one of the largest hog operations in the country with nearly 30,000 animals (Ludwig et al. 2003). Despite being an Islamic country, annual pig production had risen significantly in the decade prior to the outbreak, from less than 3 million head to more than 5 million (FAO 2007b).

Like other contagious respiratory viruses, Nipah virus is presumably a density-dependent pathogen, requiring a certain threshold density of susceptible individuals to spread, persist, and erupt from within a population (Newman et al. 2005). Analyzing the management data from the index farm, Olival and Daszak (2005) concluded that the population size and stocking density of the pigs, combined with the continual influx of young, immunologically na"ive pigs, may have facilitated the adaptation and amplification of the virus.

Nipah virus shares similarities with Hendra virus, another paramyxovirus, identified in Australia after infection resulted in the deaths of two people handling horses thought infected by flying foxes (Field et al. 2001). No evidence of prior Hendra virus infection was found in a serologic study of wildlife rehabilitators who had had frequent, sustained, and significant contact with these bats, however (Selvey et al. 1996). This suggests a requirement for adaptation and/or amplification in an intermediate host for these viruses, though a case-control study of patients in a resurgence of Nipah virus disease in Bangladesh = starting in 2004 linked infection with the consumption of fresh, raw, date palm sap thatwas likely directly fruit-bat contaminated (Luby et al. 2006). Phylogenetic analysis suggests that Nipah and Hendra are not new viruses (Gould 1996), but remained uncharacterized until ecological changes precipitated contact with susceptible hosts.

\section{B. Hemorrhagic Fevers}

The 1992 Institute of Medicine report details how new selection pressures can give rise to new zoonotic viruses (Lederberg et al. 1992). Stefan Pattyn, the editor of Ebola Virus Haemorrhagic Fever (1978), wrote: "The larger the scale of man-made environmental changes, the greater must be the probability of emergence of a zoonosis, old or new." There are few changes greater than climate change.

Like Nipah virus, the emergence of the Sin Nombre virus in NewMexico in 1993 was also linked to changes in host reservoir food supplies brought on by El Nirno events (Glass et al. 2002) that were thought linked to climate change (Trenberth and Hoar 1996). Global warming may also affect the latitudinal and elevational movement of vectored diseases such as malaria, dengue fever, and West Nile 
virus encephalitis (Haines and Patz 2004). One scenario-based modeling study suggested that by 2100 , the few-degree mean global temperature increase could expand the zone in which malaria is spread from $45 \%$ of the world's population to $60 \%$ (Martens et al. 1995). The emissions and loss of forest cover associated with slash-and-burn deforestation contribute to the problem, especially if then used as grazing land, as the livestock sector has been estimated to contribute more $\mathrm{CO}_{2}$ equivalents of greenhouse gases than global transport (Steinfeld et al. 2006).

As leading cattle-producing nations fought during World War II, Argentina dramatically expanded its beef industry at the expense of its forests. The ensuing upsurge in field mouse populations likely led to the surfacing of the Junin virus in the early 1950s (Hoff and Smith 2000) nowplacing an estimated 5 million people are now at risk for Argentine hemorrhagic fever infection (Enria and Feuillade 1998). Neotropical deforestation for agricultural use has since been associated with the emergence of other Biosafety Level 4, rodent-borne, hemorrhagic fever arenaviruses: Machupo virus in Bolivia, Sabia virus in Brazil, and Guanarito virus, the cause of Venezuelan hemorrhagic fever (Charrel and de Lamballerie 2003). Inroads into Afrotropical rainforests exposed other hemorrhagic fever viruses: Lassa, Rift Valley Fever, and Ebola. All known hemorrhagic fever viruses are suspected to be zoonotic in nature (Guenno 1997).

Deforestation may also lead to a surge in other zoonotic pathogen vectors. Many disease-carrying mosquitoes prefer to breed in the open along partially cleared forest fringes, rather than deep in the woodland (Sutherst 2004). A recent study of the primary vector of Falciparum malaria in the Peruvian Amazon found that mosquito-biting rates in roadside, deforested settlements were up to 278 times higher than those in settlements without adjacent deforestation (Vittor et al. 2006). When livestock are grazed on the cleared land, they may in turn nourish and amplify arthropod vectors (Patz and Wolfe 2002).

In India, what was initially thought to be a severe outbreak of yellow fever in 1957 was in fact a novel disease named after the recently deforested Kyasanur forest and caused by a tick-borne flavivirus (Taylor 1997). The cleared land was used for the grazing of cattle, a major host for the tick species (Haemaphysalis spinigera) that carried the virus out from its simian reservoir to now cause as many as 1,000 human cases each year (Varma 2001).

\section{Lyme Disease}

Encroachment into natural habitats may bring humans into new contact with pathogens, or, in the case of Nipah virus and the reemergence of vampire bat-borne rabies in the Amazon Basin (Schneider et al. 2005), encroachment may flush pathogens out to us. In Connecticut, where Lyme disease was first recognized in 1975, encroachment might be more properly termed "suburbanization."

The density of infected, nymphal, black-legged ticks (Ixodes scapularis), the primary risk factor for Lyme disease, has been linked to increasing forest fragmentation in periurban areas, likely due to corresponding increased numbers of the white-footed mouse (Peromyscus leucopus), the principal reservoir of the Lyme bacterium (Borrelia burgdorferi) (Allan et al. 2003). This "edge" habitat effect on $P$. leucopus density is thought due to decreased predation and competition in small forest fragments interspersing residential development (Nupp and Swihart 1996). Brownstein et al. (2005), though, compared human disease incidence with habitat fragmentation using satellite imagery of landscape forest patches and showed that entymological risk does not necessarily translate into increased human risk, perhaps because, at a certain level of suburban development, fewer residential properties adjoin woodlots (Maupin et al. 1991).

\section{BUSHMEAT}

Humans and our ancestors have likely consumed bushmeat, wild animals killed for food, for millions of years (Boesch 1994). During the 20th century, however, commercial hunting using firearms and wire snares to supply logging (Wilkie et al. 1992) and oil exploration (Thibault and Blaney 2003) operation concessions along new roadway networks has dramatically increased the catch in Central African forests (Fa and Garc'ia-Yuste 2001). Annually, it is estimated that 579 million wild animals are caught and 
consumed in the Congo basin, equaling 4.5 million tons of bushmeat, with the addition of a possible 5 million tons of wild mammalian meat from the Amazon basin (Fa et al. 2002).

Tropical lowland forest habitat contains the world's greatest terrestrial biodiversity (Turner 1996) and may therefore harbor a reservoir of zoonotic pathogens. Logging in Central Africa generally involves selective extraction of high-value timber species rather than clear-cutting, which may maintain this higher natural density of potential hosts (Fa et al. 1995). Karesh and Cook (2005) estimate that the wildlife trade in general generates in excess of one billion direct and indirect contacts between humans and domesticated animals annually. The broad range of tissue and fluid exposures associated with the bushmeat industry's hunting and butchering may make these wildlife interactions especially risky (Wolfe et al. 2000).

Hunting has been associated with the contraction of diseases such as contagious pustular dermatitis, a disease caused by the parapoxvirus Orf virus (Wolfe et al. 2000), and monkeypox, which is associated with the preparation and consumption of infected rodents (Pattyn 2000). In Africa, as many as 30 different species of primates are also hunted and processed by the bushmeat industry (Peeters et al. 2002). Conservation concerns aside (Walsh et al. 2003), primate butchering and consumption may present particular risk of cross-species disease transmission. The hunting of red colobus monkeys, for example, was implicated in a localized epidemic of human monkeypox that persisted for four generations of probable human-to-human transmission (Jezek et al. 1986).

Evolutionary distance may explain why humans are more likely to get "mad cow disease" than Dutch elm disease. Near-universal taboos against cannibalism may have well-served the human species by reducing enteral and parenteral exposure to well-adapted viruses (Karpowicz et al. 2004), but full sequencing of the chimpanzee genome has revealed how closely humans are related to other anthropoid primates (Gunter and Dhand 2005).

By consuming other members of the family Hominidae, humans may be exposed to pathogens sharing similar host adaptations (Brack 1987). For instance, human outbreaks of Ebola hemorrhagic fever have been traced to exposure to the dead bodies of infected chimpanzees (WHO 1996) and gorillas (WHO 2003) hunted for food. Indeed, the primary risk factor found for Ebola virus infection among wild chimpanzees was hunting behavior (Formenty et al. 1999), which involves not only the consumption of raw meat, but a rudimentary butchering with mouth and hands (Wolfe et al. 2000). Ebola virus is the cause of one of humanity's deadliest infections, but is not efficiently spread (Legrand et al. 2007) compared to a virus like HIV.

\section{A. HIV}

The year the global eradication of smallpox was declared (WHO 1980b), the first reported cases of AIDS were being identified (CDC 1981). Since then, HIVIAIDS has resulted in an estimated 65 million infections and 25 million deaths. In several African countries, the estimated prevalence of HIV now exceeds $20 \%$ of the adult population (UNAIDS 2006).

Although blood banking, the relaxation of sexual mores, and injection drug use facilitated the spread of HIV, the simplest and most plausible explanation for the emergence of the virus appears to be exposure to animal blood or excretions as a result of hunting and butchering primates, or the subsequent consumption of uncooked or contaminated bushmeat (Sharp et al. 2001).

Blood samples obtained from 573 freshly butchered primates in logging concessions and bushmeat markets found $18.4 \%$ with evidence of Simian Immunodeficiency Virus (SIV), considered the precursor to HIV (Peeters et al. 2002). Although HIV-like viruses have been recently discovered in gorillas, the butchering of chimpanzees is considered the most likely source for HIV-1, the strain of the AIDS virus that has spread around the world (Van Heuverswyn and Peeters 2007).

Molecular analyses now suggest SIV crossed species not once, but on no fewer than eight separate occasions in recent history (Peeters et al. 2002). Until a few decades ago, such a zoonotic transmission event may have only affected a small number of isolated rural villages. Corporate logging for export 
expanded western Africa's tradition of primate subsistence hunting (Hahn et al. 2000) into a major commercial enterprise (Larkin 2000) that extends into surrounding major cities where ape meat garners premium prices in restaurants (Weiss and Wrangham 1999). Combined with reports of the frequent rotation of sex workers into logging camps by timber companies (Nisbett and Monath 2001), the routes of transmission along roads cut by deforestation in Africa may run both ways (Peeters 2004).

In addition to concurrent socioeconomic disruptions of post-colonial, sub-Saharan African infrastructure, widespread iatrogenic and self-injection use of unsterile needles may have played a role in the crossspecies adaptation of the virus and account in part for the simultaneous appearance of multiple strains of HIV across Africa (Marx et al. 2001). Similar possible iatrogenic facilitation of infection has been reported with parenteral, antischistosomal vaccination in Egypt, which may have led to widespread hepatitis C virus distribution (Frank et al. 2000), as well as the exposure of millions of Americans to simian virus 40 in batches of polio vaccine between 1955 and 1963 (Stratton et al. 2002).

While zoonotic diseases such as rabies may result in the deaths of approximately 50,000 people globally every year (Meslin et al. 2000), for most zoonoses, humans are the dead-end host (Woolhouse and Gowtage-Sequeria 2005). In terms of global public health implications, the greatest concern surrounds zoonotic infections like HIVIAIDS that can not only jump from animals to humans, but can then spread human-to-human. More people engage in sexual activity than the butchering of chimpanzees.

Emerging retroviruses are of special concern because of their ability to insinuate into host DNA. There are seven genera in the Retroviridae family: lentivirus, including HIV and SIV, spumavirus, and five groups of cancer-causing retroviruses previously clustered together as oncovirus (Knipe and Howley 2007). SIV is just one of a large reservoir of poorly characterized lentiviruses in African primates raising the specter of additional AIDS-like zoonoses arising from continued bushmeat consumption (Peeters et al. 2002).

Retroviruses are also highly recombinogenic (Hu and Temin 1990). Given the high prevalence of HIV in Central Africa (UNAIDS 2006), a vulnerable pool of immunosuppressed hosts might not only facilitate the establishment of novel pathogens, but act as a retroviral mixing vessel for the recombination of HIV with other primate lentiviruses. This could potentially result in changes in HIV drug resistance, tropism, or virulence (Chen et al. 2006b).

\section{B. Other Primate Retroviruses}

\section{SFV}

In 1971, a spumavirus was isolated from a nasopharyngeal carcinoma in a Kenyan patient (Achong et al. 1971) sharing 95-97\% amino acid homology with a strain found in chimpanzees whose natural habitat includesKenya (Schweizer and Neumann-Haefelin 1995). This suggested zoonotic transmission, but laboratory contamination could not be ruled out (Meiering and Linial 2001). Subsequent case reports of laboratoryworkers exposed to primate fluids have shown evidence of persistent infection with the spumavirus simian foamy virus (SFV), confirming human susceptibility to other classes of primate retroviruses (Heneine et al. 1998).

Cameroon is representative of a region in Africa in which a noteworthy array of new zoonotic pathogens has been identified, including HIVIAIDS, Ebola and Marburg viruses, and monkeypox. The country is home to among the highest density of large anthropoid primates in Africa, as well as high humanexposure frequency to wild primate tissues from hunting (Wolfe et al. 2000). Indeed, in one survey of rural villagers involved in a community-based HIV-prevention campaign in Cameroon, $61 \%$ of participants reported direct exposure to fresh, non-human primate blood and body fluids (Wolfe et al. 2004), making this area well-suited for a study of the zoonotic potential of SFV in bushmeat.

Ten out of 1,099 (1\%) Cameroonese adults sampled had antibodies to SFV, suggesting that primate retroviral zoonoses were more frequent, widespread, and contemporary than previously appreciated (Wolfe et al. 2004). Road networks now connecting such remote populations with urban access to 
international travel could allow for global dissemination of any emerging bushmeat-borne zoonoses (Robbins et al. 2003).

Although SFV infection is enzootic in many wild primate populations, seroprevalence is generally higher in primates housed in captivity, approaching $100 \%$ in some studies (Meiering and Linial 2001). Schweizer et al. (1995), for example, found $93 \%$ of captive African green monkeys infected with SFV, compared to a separate study in which $36 \%$ of wild monkeys tested positive (Stiles 1968). Given this propensity, animal handlers from primate research centers and zoos in the United States were serologically screened to further gauge the extent of SFV spread.

Ten new SFV-infected persons were identified out of the 187 lab and zoo workers studied (5.3\%) (Switzer et al. 2004). Since fewof the primate contacts involved blood exchanges, saliva was considered the likely route of transmission. A later study found that entertainment workers could also be at risk of infection, as SFV was detected by PCR in $52.9 \%$ of a small sample of performing macaques in Indonesia (Schillaci et al. 2005). Primate pet owners and tourists visiting "monkey temples" in Asia also risk exposure (JonesEngel et al. 2006).

The persistence of infection is characteristic of foamy viruses, with recovery of infectious SFV from a human host demonstrated 20 years after zoonotic transmission, despite high antibody titers (Schweizer et al. 1997). Putative foamy virus disease associations in humans have been made with dialysis encephalopathy (Cameron et al. 1978), de Quervain' thyroiditis (Werner and Gelderblom 1979), multiple sclerosis (Westarp et al. 1993), sensorineural hearing loss (Pyykko et al. 1994), familial Mediterranean fever (Tamura and Kira 1995), myasthenia gravis (Liu et al. 1996), and Graves' disease (Lee et al. 1998), but no definitive links have been found (Meiering and Linial 2001).

Two AIDS patients dying after baboon liver transplants showed PCR evidence of baboon foamy virus sequences in tissues distant to the transplant (Allan et al. 1998), but infection of human cells was not specifically analyzed, raising the possibility that the viral sequences found were due to migrating donor cells (i.e., leukocytes). Without a study of SFV infection in immune-compromised hosts, supposition that SFV has a more aggressive course in an immunologically permissive environment will remain speculative (Jones-Engel et al. 2005).

Spouses of SFV-exposed animal handlers show no evidence of infection, suggesting the virus is not readily spread human-to-human (Switzer et al. 2004). More recently, however, SFV infection was described in a Central African sex worker (Switzer et al. 2005). The prevalence of SFV infection among lab and zoo workers has raised concerns about blood supply safety. The demonstration of SFV transmission via experimental whole-blood transfusions in primates has led to the consideration of excluding those with ongoing occupational primate exposure from the blood donor pool (Brooks et al. 2007).

\section{HTLV}

Further analysis of the Cameroon blood samples also revealed evidence of emerging zoonotic transmission of a third class of human retroviruses, deltaretrovirus (Wolfe et al. 2005). Two strains of human T-lymphotropic virus (HTLV), types 1 and 2, were already known to infect an estimated 15 to 20 million people globally, spread sexually, vertically, or parenterally, via blood transfusion or IV drug use (Mahieux and Gessain 2003). Also known as human T-cell leukemia/lymphoma virus, HTLV-1 can have a long latency period before triggering an aggressive hematological malignancy in 1 in 1,500 adult HTLV-1 carriers per year (CDC 1987). Both HTLV-1 and HTLV-2 are also associated with a progressive neurodegenerative disease known as tropical spastic paraparesis (Roucoux andMurphy 2004) and are thought to have an ancient zoonotic primate source (Courgnaud et al. 2004).

Wolfe et al. (2005) first identified HTLV-3 and HTLV-4 in Cameroon monkey and ape hunters. These findings suggest that retroviruses continue to cross actively into human populations, though whether HTLV-3 and HTLV-4 are pathogenic or can be passed between humans remains to be shown (Kuehn 2005). 
Because the latency period of viruses like HTLV can be decades (Blatter 1989), there is concern that emerging primate retroviruses could circulate in the human population years before discovery, at which point it may be too late to stop an epidemic (Fricker 2000). Although improved global public health surveillance and blood supply screening may aid early detection, efforts to curtail the commercial bushmeat trade might reduce the frequency of nascent zoonotic transmission.

\section{SARS}

The expansion of the bushmeat trade in Africa has been well-documented (Barnes 2002), but a similar surge in Asia has received less attention (Bennett and Rao 2002). The intensive commercial bushmeat trade likely got its start in Asia (Bell et al. 2004), particularly in Guangdong, the southern Chinese province surrounding HongKong SAR and presumed birthplace of both the highly pathogenic avian influenzavirus A subtype H5N1 (H5N1) (Chen et al. 2004) and the epidemic of atypical pneumonia referred to as severe acute respiratory syndrome, or SARS (Donnelly et al. 2003).

A novel coronavirus, SARS-associated coronavirus (SARS-CoV), was identified (Peiris et al. 2003). Before SARS, Coronaviridae were typically only known to cause a mild, common cold-like illness in humans (Siddell 1995), though they are a common occurrence in a range of avian and mammalian species and include turkey, feline, and canine enteric coronaviruses; infectious bronchitis virus in chickens; porcine hemagglutinating encephalomyelitis virus; porcine and bovine enteric and respiratory viruses; and murine hepatitis virus (Holmes 1999).

The preponderance of persons handling, killing, or selling caged, wild animals used for food among early index SARS cases in Guangdong (Xu et al. 2004), combined with the detection of SARS-CoV-like viruses in both animals and workers at Chinese live animal markets, suggested the market system played a role in the amplification and transmission of the virus (Guan et al. 2003). A subsequent survey of animals in retail markets focused attention on the cat-like masked palm civet (Paguma larvata) (Guan et al. 2003).

In China, animals are eaten for enjoyment, sustenance, and for their purported medicinal qualities (Lawrie 2004). In addition to civets being trapped and raised for meat, civet penis is consumed as an aphrodisiac (Bell et al. 2004) and coffee beans fed to captive civets are recovered from their excreta, flavored with perianal gland scent (William 2003).

The search for the original zoonotic reservoir of SARS has narrowly honed in on wildlife farms, animal markets, and restaurants in China without recognition that many of the wild animals sold are smuggled into the country via an expanding regional black market trade (Bell et al. 2004). Roberton et al. (2003) documented a vast expansion of the wildlife trade in the 1990s, in which the commercial sale of exotic species to supply the emerging urban middle-class demand largely supplanted subsistence hunting. Many of the wild animals, typically while still alive, enter China through Vietnam from Lao PDR where the bushmeat trade rose to become the second-largest income source for rural families (Duckworth et al. 1999). Studying a single Laotian market, Srikosamatara et al. (1992) estimated the per-annum sale of 8,000 to 10,000 animals of at least 23 mammalian species, 6,000 to 7,000 animals of more than 33 avian species, and 3,000 to 4,000 reptiles, for a total weight of 33,000 kilograms.

With growing populations and increasing demands for wildlife meat outstripping local supplies, the transboundary wildlife trade has been supplemented with the creation of intensive, captive production farms in which wild animals may be raised under poor sanitation in unnatural stocking densities (Karesh et al. 2005) before being transported and caged at markets for sale. Selling animals while still alive guarantees freshness in the minds of many regional consumers, but may result in potential pathogen discharge into the retail setting (Brown 2004). Together, these factors have been implicated in the amplification of emerging zoonotic pathogens with the potential to infect hunters, farmers, and market patrons (Gilbert et al. 2004).

Seroprevalence studies have found that while civets at farms that supply the live markets were largely free of infection, up to $80 \%$ of those at markets harbored significant antibody titers to SARS-CoV (Tu et al. 2004). This suggests that most infections happened at the market, perhaps because of a combination 
of crowded interspecies mixing and the immunosuppressive effect of stress (Padgett and Glaser 2003). Following the SARS outbreak, the Chinese government reportedly confiscated more than 800,000 wild animals from the markets of Guangdong.

Recent data suggest that the masked palm civet is merely an intermediate host transmitting SARS-CoVlike viruses to humans from their natural host reservoir, bats ( $\mathrm{Li}$ et al. 2005), which are also eaten in Guangdong Province (Woo et al. 2006). Just as bats are likely the original source of all terrestrial carnivore rabies (Lyssavirus) viruses (Bardrane and Tordo 2001), they may also be the starting point for all coronavirus lineages (Vijaykrishna et al. 2007).

Analogous to the role pigs played in Nipah virus disease, civets may facilitate transmission of SARS-CoVlike viruses by acting as an amplifying host with frequent human contact, but may be more than just passive conduits. Genomic sequencing of SARS-CoV-like viruses in palm civets over time suggests not only viral adaptation (increased viral loads) to the civets (Song et al. 2005), but perhaps adaptation to humans as well.

The SARS-CoV spike (S) protein has been shown to be responsible for virus attachment to susceptible cells (Hofmann et al. 2004), and two key amino acid residues were identified as critical for speciesspecific receptor binding (Qu et al. 2005). Using pseudotyped lentiviruses, Qu et al. (2005) demonstrated that substituting both residues in the human SARS virus with those found in civet isolates nearly abolished human cell infectivity, and single substitutions reduced receptor binding affinities. Conversely, the reverse double substitution in the civet virus enabled it to infect human cells.

After the initial SARS epidemic ended in July 2003, four new human cases were confirmed in Guangdong that winter. Unlike most of the previous cases, they had not had close contact with infected persons and presented with mild clinical symptoms. Epidemiologic and phylogenetic analyses indicated that these cases likely represented a second, independent zoonotic transfer. Viral isolates from palm civets at both a local market and the restaurant with which three of the four human cases were associated were sampled at the time and were nearly identical to those found in the new, milder human cases. Civet isolates were detected that shared one of the two civet-to-human $S$ protein critical residue substitutions found in all of the new human patients and none of the prior year's civet isolates. The findings suggested a cross-host "exaptive" adaptation to civets may have resulted in greater human infectivity (Song et al. 2005).

Following the reemergence of SARS cases, Kan et al. (2005) conducted a more comprehensive phylogenetic analysis of SARS-CoV-like viruses in market civets and concluded that the evolutionary starting point for SARS-CoV was a prototype group of viruses exclusive to nonhuman animals that gave rise to the low pathogenicity group responsible for the later sporadic, mild human cases, which in turn led to a highly pathogenic grouping of viruses responsible for the 2003 epidemic. Based on this molecular epidemiology data, Zhao (2007) suggested that the palm civet may serve not only as transitional host and amplifier of SARS-CoV-like viruses, but a "favorable incubator" as well.

Interestingly, genomic and molecular clock analyses suggest that the bovine coronavirus now causing shipping fever disease jumped species around the year 1890, resulting in the emergence of human coronavirus OC43 (Vijgen et al. 2005). This tempted Vijgen et al. to conjecture that the 1889-1890 pandemic ascribed to influenza may have been the result of a SARS-like interspecies transmission event triggered by the reported mass culling of cattle herds between 1870 and 1890, an extensive depopulation associated with a highly pathogenic bovine respiratory disease panzootic.

\section{LIVE ANIMAL TRANSPORT}

Culinary choicewas likely not the only key factor in the emergence and spread of SARS-CoV. Like HIV, historical zoonotic transmission and adaptation of SARS-CoV-like coronaviruses may have died out in a remote rural setting. Instead, given today's unprecedented speed, volume, and reach of global air travel, a worldwide outbreak of SARS was seeded from a single person on a single day on a single floor of a Hong Kong hotel (MacKenzie et al. 2004). That index case, a physician from Guangdong, had attended a wedding. As guests departed, the virus coughed by one man spread to five countries within 24 hours (Lee 
and Krilov 2005).Within months, the virus spread to 30 countries on six continents (Mack 2005), causing 8,096 probable cases and 774 deaths(WHO2004). In the past, a trip around the world took a year; today, we and our viral baggage can circle the globe in 24 hours (Johnson 2003). The annual number of globetrotters now exceeds an estimated 1 billion people (Lashley 2004).

\section{A. Exotic Pet Trade}

Humans are not the only global travelers, and zoonotic vectors do not make their way out of jungles solely as bushmeat. Indeed, the first filovirus discovery was not in Africa, but 4,000 miles away in Marburg, Germany, causing an outbreak that had its origins in a laboratory that received monkeys from Uganda (Martini 1969). Likewise, Ebola virus, from the same family Filoviridae, was imported into the United States in monkeys destined for research (Jahrling et al. 1990). Holding different populations of captive primates together during transport or while housed at or en route to zoos or laboratories might result in viral recombination, potentially resulting in unpredictable, new strains that could otherwise never have occurred (Chapman et al. 1995).

Animals translocated for other human uses have also been implicated in the spread of zoonotic disease. The international movement of horses for equestrian competitions has been associated with the spread of equine influenza (Powell et al. 1995; Guthrie et al. 1999). After their introduction into Eastern Europe and New Zealand, fur-bearing animals became new reservoirs for rabies (Gylyset al. 1998) and M. Bovis (Woodford and Rossiter 1993), respectively. Rabies was ferried into the mid-Atlantic United States in the 1970s when hunting pens were repopulated with raccoons trapped in enzootic zones of the South (Woodford and Rossiter 1993), and the movement of hares from central and Eastern Europe for sporting purposes has led to several outbreaks of tularemia (Godfroid et al. 2005). Swapping the wings of Aedes aegypti for those of a jumbo jet, the dengue flavivirus has triggered major intercontinental outbreaks of dengue fever traced to inadvertent live animal transport of mosquitoes and their eggs (Reiter and Sprenger 1987).

Whether for exotic cuisine or exotic pets, animals imported together may end up in holding areas in dealer warehouses, where they and their viruses can further interact. The first human outbreak of monkeypox in the Western hemisphere, resulting in 71 cases across six Midwestern U.S. states (Gross 2003), was traced to monkeypox-infected Gambian giant rats shipped to a Texas animal distributor along with 800 other small mammals snared in Ghana. Notably, there were no infections recorded directly from the African rodents; exposure was derived from secondary and tertiary contacts often inherent to the trade (Chomel et al. 2007).

A similar situation exemplifying the multiplicity of interspecies contacts was thought to have transpired in 2005 when the British government announced that H5N1 virus had been detected in an imported parrot. This followed a case the year before of a Thai man stopped for a routine random drug check in a Belgian airport and discovered to be smuggling a pair of rare crested hawk eagles stuffed into plastic tubes in his luggage. Both of the birds were found to be H5N1-infected (Borm et al. 2005). The parrot, however, had been imported from H5N1-free Suriname. The working hypothesis was that the bird contracted the virus while housed in a quarantine facility with finches from Taiwan found to be infected, though Taiwan had similarly been declared bird flu-free. Perhaps infected birds had been smuggled from China into Taiwan for global export, infected the Taiwanese birds, which were then legally imported into the U.K. to mix with and infect the South American parrot (DEFRA 2005). (The British Government later admitted a procedural laboratory error in which the parrot and finch samples were accidentally pooled, calling the psittacine infection into question (Redrobe 2007), but the mistake still does not explain how the finches had first become infected.) Indeed, that same month, a freighter reportedly had been caught trying to smuggle into Taiwan from mainland China more than 1,000 birds, some infected withH5N1 (COA 2003).

Pet bird shipments have also been implicated in outbreaks of psittacosis (Moroney et al. 1998) and their involvement speculated in the introduction ofWest Nile virus from the Middle East (Lanciotti et al. 1999) into the Western hemisphere (Rappole et al. 2000). Herpes B virus is enzootic in macaques (Weigler 1992) yet is a rare though often fatal cause of human fulminating meningoencephalitis (Holmes et al. 1995), which, combined with reports of rabies exposure (Favoretto et al. 2001), raises concerns about 
people keeping primates as pets (Ostrowski et al. 1998). African pygmy hedgehogs have triggered salmonellosis in the Americas (Riley and Chomel 2005), and terrapins shipped from the Americas have caused salmonellosis in Ireland (Lynch et al. 1999).

Worldwide, an estimated 40,000 primates, 4 million birds, 640,000 reptiles, and 350 million tropical fish are traded live each year for the pet trade (Karesh et al. 2005). Wildlife trafficking in exotics is estimated to be a 6 billion USD industry (Check 2004).

\section{B. Livestock Transport}

Over the last century, there has been a shift away from livestock production as a highly localized enterprise, where animals were typically born, fattened, and slaughtered in the same region. The number of live animals traded for food quintupled in the 1990s, with more than one billion moved across borders in 2005 (FAO 2007b). Long-distance live animal transport may make countries more vulnerable to acts of bioterrorism with zoonotic agents (GAO 2005), a risk thought amplified by the concentrated and intensive nature of contemporary industrial farming practices (Chalk 2004). The transport and crowding of animals from different herds or flocks in poorly ventilated and stressful environments for long periods has been considered ideally suited for spreading disease (FAO 2002).

The immunosuppressive stress of prolonged live animal transport may not only increase a healthy animal's susceptibility to infection, but may trigger the emergence of a variety of diarrheal and respiratory diseases caused by endogenous micro-organisms that might not normally lead to disease. So-called "shipping fever," for example, the bovine version of which costs U.S. producers more than 500 million USD a year, is often caused by latent pathogens that may become active when transporting cattle long distances (Roth and Flaming 1990). Approximately 3,000 truckloads of cattle are moved per day in the United States (Crews 2004).

Long-distance transport may also increase the fecal shedding of zoonotic disease agents. Barham et al. (2002) found the average prevalence of Salmonella within feces and on the hides of cattle to be $18 \%$ and $6 \%$, respectively, before transport. After the animals were loaded onto a vehicle and trucked for 30 to 40 minutes, Salmonella levels found in feces increased from $18 \%$ to $46 \%$, and the number of animals with contaminated hides escalated from $6 \%$ to $89 \%$ upon arrival at the slaughter plant. A recent study found that from the time cattle were loaded onto tractor-trailers at the feedlot to the time their hides were removed during processing, the prevalence of the enterohemorrhagic $E$. coli O157:H7 on hides increased from $50.3 \%$ to $94.4 \%$ (Arthur et al. 2007). E. coli $\mathrm{O} 157: \mathrm{H} 7$ isolates from transport trailer walls have been genotypically matched to hide samples in the packing plant (Childs et al. 2006). Fecal pathogens on the hide may then end up in the meat supply (Arthur et al. 2007). Similar results were found in pigs (Marg et al. 2001) and chickens (Whyte et al. 2001) raised and transported for meat.

Regarding the spread of the Nipah virus inMalaysia in 1999, the Thai Minister of Public Health was quoted as saying: "A hundred years ago, the Nipah virus would have simply emerged and died out; instead it was transmitted to pigs and amplified. With modern agriculture, the pigs are transported long distances to slaughter. And the virus goes with them" (Specter 2005). One hundred people died in the Malaysian outbreak (CDC 1999), whereas avian influenza viruses are thought to have the potential to spawn pandemics capable of killing millions (Murray et al. 2006).

In early 2004, outbreaks of the highly pathogenic avian influenza virus H5N1 were reported across eight countries in Southeast Asia nearly simultaneously. Given the pattern and timing of outbreaks, the Food and Agriculture Organization (FAO) of the United Nations identified the transport of live birds reared for human consumption as a primary culprit in the rapid spread (FAO 2007a). The FAO reported, for example, that 5,000 chickens succumbed to avian influenza in Lhasa, Tibet, and that these infected birds were transported to Tibet from China's Lanzhou city-a trip spanning more than 1,600 kilometers (FAO 2004b). The further the animals are transported, the further the diseases may spread (DG-SANCO 2002).

Long-distance live animal transport has also been blamed for the spread of swine influenza viruses in the United States, where livestock may travel an average of 1,000 miles (Wilson et al. 2000). Throughout 
much of the 20th century, influenza viruses had established a stable H1N1 lineage within U.S. pigs, becoming one of the most common causes of respiratory disease on North American pig farms (Zhou et al. 1999). That seemed to have changed in August 1998 when thousands of breeding sows fell ill on a North Carolina pig farm. An aggressive $\mathrm{H} 3 \mathrm{~N} 2$ virus was recovered, bearing the $\mathrm{H} 3$ and $\mathrm{N} 2$ antigens of the human influenza strain circulating since 1968. Not only was this highly unusual, but, upon sequencing of the viral genome, researchers found that it was not just a double reassortment (a hybrid of human and pig viruses, for example), but a never-before-described triple reassortment of human, avian, and porcine influenza virus gene segments (Zhou et al. 1999), which raised concerns about further mammalian adaptation of influenza virus (Wuethrich 2003b).

The continuous cycle of U.S. mass animal movement, involving inter-auction movements and intraauction mixing, may provide a built-in dispersal mechanism for potentially zoonotic disease agents (Shields and Matthews 2003).Within months of the appearance of the new swine flu virus in North Carolina, it surfaced in Texas, Minnesota, and lowa (Zhou et al. 1999). Within one year, it had spread across the United States (Webby et al. 2000). The rapid dissemination across the nation was blamed on the cross-country transport of live pigs (Wuethrich 2003b). In the United States, pigs travel coast to coast, frequently born in North Carolina, fattened in the corn belt of lowa, and then slaughtered in California. It is often cheaper to transport the animals to the feed rather than transport the feed to the animals (USDA ERS 2003). While this regional segmentation of production stages may cut down on short-term costs for the pork industry (Burrell 2002), the highly contagious nature of zoonotic diseases like influenza, perhaps made further infectious by the stresses of transport (Wuethrich 2003b), must be considered when calculating the true cost of long-distance live animal transport.

\section{LIVESTOCK REVOLUTION}

Driven by the population explosion, urbanization, and increasing incomes, the per-capita consumption of meat, eggs, and dairy products has dramatically expanded in the developing world, leading to what has been termed the "Livestock Revolution" beginning in the 1970s, akin to the 1960s Green Revolution in cereal grain production (Delgado et al. 1999). From around 1980 to 2004, world meat production nearly doubled to 260 million tons (FAO 2006a). To meet the growing demand, livestock production may double again by 2020 (Pearson et al. 2005).

Animal agriculture worldwide is increasingly moving from the relatively low efficiency, family-centered, low-input model to intensive systems, which are loosely defined as the production of large numbers of genotypically-similar animals often under concentrated confinement with rapid population turnover. Traditional systems are being replaced by intensive systems at a rate of more than $4 \%$ per year, particularly in Asia, Africa, and South America (Pearson et al. 2005).

Intensive animal agriculture began in the United States with poultry production more than 60 years ago and has become the norm for both poultry and swine production (Pearson et al. 2005). The vast majority of chickens and turkeys raised for meat in industrialized countries, and increasingly throughout the developing world (Mason and Crawford 1993), are now typically reared in long sheds, each containing 15,000 to 50,000 birds (Otte et al. 2007).Worldwide, an estimated $70-80 \%$ (UEP 2004) of egg-laying chickens are intensively confined in battery cages, small, barren, wire enclosures often stacked several tiers high and extending down long rows in windowless sheds. It is not uncommon for egg producers to keep hundreds of thousands or even more than one million hens confined on a single farm (Mench 2002). It is estimated that half of the world's pig population, which now approaches one billion animals, are raised in industrial confinement (Nierenberg 2005).

While heralded for its efficiency and productive capacity, this industrial model has raised sustainability concerns regarding the waste absorption and feed supply capacity of available land (Delgado et al. 1999), as well as trepidation over associated zoonotic risks (WHO 1999). This intensive industrialization of animal agriculture may represent the most profound alteration of the animal-human relationship since domestication (Delgado et al. 1999). Given the emergence of some of humanity's most important diseases in the Neolithic era, there is concern that new threats may arise from the broadly significant changes currently taking place in global livestock production (Slingenbergh et al. 2004). 
Reviewing the 177 human pathogens classified as emerging or reemerging, Woolhouse and GowtageSequeria (2005) identified changes in land use or agriculture as the main driver of their appearance. Unnaturally high concentrations of animals confined indoors in a limited airspace and producing significant quantities of waste may allow for the rapid selection, amplification, and dissemination of zoonotic pathogens (Pearson et al. 2005). In the United States, the average numbers of animals in each chicken, pig, and cattle operation approximately doubled between 1978 and 1992 (Tilman et al. 2002). According to the last U.S. Department of Agriculture census, $6 \%$ of the nation's hog farms produced three-quarters of the pigs and more than $90 \%$ of the nation's egg-laying hens were reared on $2 \%$ of the country's egg farms (USDA 2002). The crowding of increasingly greater numbers of animals into increasingly smaller spaces has been identified as a critical factor in the spread of disease (Delgado et al. 2003).

Physiological stress has been associated with both confinement (Andr'e and Tuyttens 2005) and the various physical modifications visited upon farm animals without anesthesia (Puvadolpirod and Thaxton 2000), including castration, branding, dehorning, detoeing, teeth clipping, beak trimming, and tail docking (Battaglia 2001). This, coupled with the metabolic demands of intensive production, such as artificially augmented reproduction, lactation, early weaning, and accelerated growth rates, may leave animals extremely prone to disease (Cameron 2000).

The loss of genetic diversity in modern industrial livestock production may also play a role in increased disease susceptibility (USDA ARS 2006). Over the last century, an estimated 1,000 farm animal breeds have disappeared, and another 1,000 breeds are presently at risk for extinction-about one-sixth of the world's cattle and poultry varieties-in large part due to the dilution or displacement of native breeds in developing countries with breeding stock from industrialized countries (FAO 2004a).

This erosion of biodiversity may have public health implications. Genetic "bottlenecking" created by narrowly focused breeding schemes may fuel zoonotic pathogen adaptation and hinder host evolution for resistance (Ebert and Hamilton 1996). Plant biologists have long understood the devastating disease potential associated with genetic uniformity (Adams et al. 1971). In the early 1970s, for example, the U.S. corn industry developed "Tcms" corn, a highly profitable strain adapted for large-scale farming. Only after $85 \%$ of the nation's seed corn acreage was covered with the new variety did the industry realize that the strain also happened to be particularly susceptible to a rare form of leaf blight fungus that then wiped out large swaths of the U.S. Corn Belt (Schrag andWiener 1995). Emerging infections may thrive when genetically homogenous members of a single species are kept in close proximity, and the expanding application of cloning technology may further narrow the genetic base (Meredith 2004).

Genetic manipulation for production traits such as rapid growth may itself carry a trade-off in immunocompetence (Norris and Evans 2000). The "resource-allocation hypothesis" suggests that protein and energy diversion from host defense to breast muscle mass production in meat-type breeds of chickens, for example, may explain why chickens with accelerated growth are at risk for increased immune dysfunction (Gross and Siegel 1988), disease morbidity (Rauw et al. 1998), and disease mortality (Yunis et al. 2000). According to one economic analysis, transitioning to slower-growing breeds of broiler chickens with improved immunity might be expected to cost poultry consumers no more than a dollar or two a year (HSUS 2006). Given the emergence of avian influenza viruses with putative pandemic potential, this may be a small price to pay for a more disease resistant global flock. Growth/productivity and disease susceptibility have also been shown to be correlated in pigs (Hawken et al. 1998), cattle (Mangel and Stamps 2001), and dairy cows (Sinclair et al. 1999).

The amount of manure produced by high-throughput animal husbandry creates a challenge to the maintenance of hygienic standards. In the United States, farm animals produce an estimated 2 billion tons of manure each year (Loehr 1978), the weight of 20,000 Nimitz-class aircraft carriers (NN 2006). Each steer can produce 75 pounds of manure a day, potentially layering feedlots with waste (Purdue Research Foundation 2001). The disease potential of high-density production has been compared to that of cities in the Middle Ages where squalid overcrowding facilitated the sewage-borne transmission of cholera and typhoid before the Sanitary Revolution of the 19th century (Tauxe 2002). Ironically, as the 
sanitary standards of human populations subsequently rose, that of farm animal populations arguably may have fallen (Muir and Aggrey 2003).

The industrialization of animal production may lead not only to greater animal-to-animal contact, but increasing animal-to-human contact, particularly when production facilities border urban areas (Murphy 1999). Though land pressures have tended to push crops and extensive animal systems away from the developing world's growing megacities, intensive livestock operations are moving closer to major urban areas in countries such as Bangladesh. This nexus, described as the "peri-urbanization" of industrial animal agriculture (Slingenbergh et al. 2004), may provide "flash points" for the source of new diseases (Delgado et al. 2003).

\section{A. Emerging Foodborne Zoonoses}

More than five tons of animal manure are produced per human capita in the United States every year (Loehr 1978), which may affect human health directly or indirectly should it contaminate food or water sources. An example of indirect harm is the emergence of a novel toxicosis caused by Pfiesteria piscicida linked to poultry manure runoff (Mahy and Brown 2000). This toxic dinoflagellate has been implicated in the deaths of an estimated one billion fish and skin lesions and neurological deficits reported in boaters, swimmers, and Pfiesteria researchers (Burkholder and Glasgow 1995).

A more direct harm can result when livestock manure contaminates municipal water supplies (Hrudey et al. 2003). In 1993, cattle effluent upstream of a failing water treatment plant supplying Milwaukee may have resulted in a record estimate of 403,000 cases of gastroenteritis caused by the zoonotic protozoan Cryptosporidium (Mac Kenzie et al. 1994), although subsequent evidence suggested a human rather than bovine fecal source (Sulaiman et al. 1998). Manure-contaminated irrigation water was presumed to be the source of the largest recorded outbreak of Escherichia coli $\mathrm{O} 157: \mathrm{H7}$, affecting more than 7,000 Japanese schoolchildren who had eaten contaminated sprouts in 1996 (Michino et al. 1999).

Animal manure has been found to be the source of more than 100 zoonotic pathogens (Walton and White 1981) that may directly contaminate the food supply. Between the two decades from 1975, when the dean of Yale's School of Medicine famously told students that there were "no new diseases to be discovered" and 1995, 17 new foodborne pathogens were identified (Liang 2002). Approximately half of all known foodborne pathogens may have been discovered within the past 25 years (Tauxe 2002).

In industrialized countries, the incidence of reported infectious food- and waterborne illnesses has more than doubled since the 1970s (Waltner-Toews and Lang 2000). An estimated 76 million Americans are stricken annually (Mead et al. 1999). Worldwide, foodborne microbial disease carries an annual estimated mortality of 20 million, with animal products topping the list of causes (Delgado et al. 1999).

The global rise in incidence in foodborne diseases has been attributed to both the greater consumption of animal-origin foods and the methods of intensive production required to supply such a demand (Adams and Motarjemi 1999). In the IOM report on emerging infections, cattle feedlots and large-scale poultry production were specifically singled out (Lederberg et al. 1992). In the United States, the majority of the 9.3 billion chickens raised each year (FAO 2007b) are typically stocked in densities between 14 and 23 birds per square meter (Fairchild 2005; UEP 2006). In addition to higher potential zoonotic disease risk in congested poultry operations (Shane 2000), higher stocking densities in other species may be associated with elevated risk of livestock infection with a number of potential zoonotic parasites and pathogens:Yersinia enterocolitica in goats (Lanada et al. 2005); Trichostrongylus in sheep (Thamsborg et al. 1996); M. bovis (White and Benhin 2004), Brucella (Salman and Meyer 1984), Salmonella (Jones et al. 1983), Neospora (Sanderson et al. 2000), and Cryptosporidium in cattle (Atwill et al. 1999); E. coli O157:H7 in both sheep and cattle (Stacey et al. 2007); Ostertagia in calves (Nansen et al. 1988); and Oesophagostomum (Thamsborg et al. 1999), Aujeszky's disease virus, and swine flu virus in pigs (Maes et al. 2000).

The greatest stocking density in animal agriculture is likely found in aquaculture, where tanks may hold up to one ton of live weight in nine cubic meters of water (Kite-Powell 2004) and floating cages have been 
known to hold up to four times this density (Chapman 1992). The dense monoculture, poor water quality, and resultant physiologic stress inherent to the "Blue Revolution" rise in industrial aquaculture in the 1980s (FAO 2000) may have played a role in the emergence of the newly discovered zoonotic pathogen Streptococcus iniae (Smith et al. 2001), which started devastating fish stocks on intensive fish farms around theworld with epidemics of acute meningitis (Goh et al. 1998). The first case series of confirmed spread to humans handling infected fish was reported in 1995: four cases of bacteremic cellulitis and one case of endocarditis, meningitis, and probable septic arthritis (Weinstein et al. 1997). Wild fish mortalities observed proximate to mariculture S. iniae epidemics suggest a risk to wildlife as well (Zlotkin et al. 1998). The rapid expansion of intensive fish farming has also been associated with an escalation in human Mycobacterium marinum infection and disease (Lehane and Rawlin 2000).

\section{Enterohemorrhagic E. coli}

Humans play little role in the epidemiology of E. coli O157:H7. It causes no signs of illness in its presumed natural host, cattle (Dean-Nystrom et al. 1998), but in humans, has a relatively low infectious dose and may result in hemorrhagic colitis and hemolytic uremic syndrome (Karmali et al. 1983). As a commensal bovine bacterium, why did E. coli 0157:H7 and the prophage it carries acquire the code for verotoxin production, in part responsible for the morbidity and mortality of colonized humans? Bovine intestinal cells have receptors for the toxin (Menge et al. 2004), which in turn may promote intestinal colonization (Robinson et al. 2006) via attaching and effacing lesions (Sheng et al. 2006). This may be another example, as perhaps is the case with SARS and civets, of pathogen exaptation, in which adaptation to a nonhuman host may increase zoonotic potential.

E. coli O157:H7was first described as an emerging foodborne zoonotic pathogen in 1982, associated with consumption of undercooked beef (Riley et al. 1983). The rise in E. coli O157:H7 as a human pathogen has been attributed to two primary factors: the demand-driven intensification of beef production and the widespread use of antibiotics in animal agriculture. Indeed, studies in Canada (Valcour et al. 2002), Sweden (Kistemann et al. 2004), and France (Haus-Cheymol et al. 2005) have positively correlated regional cattle density with the rates of human infection, and a number of antimicrobial agents have been shown to enhance E. coli $\mathrm{O} 157: \mathrm{H} 7$ toxin production, as well as induce the release of verotoxin-phages (Kohler et al. 2000), which may have enhanced environmental stability compared to their bacterial host (Moce-Llivina et al. 2003).

\section{Listeria Monocytogenes}

In 2002, E. coli O157:H7 resulted in one of the largest meat recalls in U.S. history-19 million pounds of beef (USDA FSIS 2002a) —eclipsed only three months later with the recall of 27 million pounds of fresh and frozen poultry products contaminated with Listeria monocytogenes (USDA FSIS 2002b). Although first identified in 1924 (Murrayet al. 1926), clinical Listeria isolates were a laboratory rarity until the late 1970s when reports increased in frequency and a series of human epidemic outbreaks in the 1980s in North America (Fleming et al. 1985) and Europe (Bille 1990) solidified its new status as an important foodborne pathogen associated with ready-to-eat processed meat and dairy products (Rocourt 1996).

L. monocytogenes has been described as having a "Jekyll and Hyde" personality (Gray et al. 2006). In the natural environment, it exists as a benign saprophyte living off decaying vegetation (Fenlon 1999). In humans, it is one the deadliest bacterial infections currently known, with a mean mortality rate of $20-30 \%$ or higher despite early antibiotic treatment (Schuchat et al. 1991). An invasive intracellular pathogen causing granulomatosis infantiseptica in term fetuses (Klatt et al. 1986), meningitis in neonates (Synnott et al. 1994), and meningoencephalitis in adults (Lorber 1996), L. monocytogenes may be responsible for $28 \%$ of deaths due to foodborne illness in the United States (Mead et al. 1999).

What is responsible for this transformation from an environmental soil bacterium to public health hazard? When Listeria cells are ingested by livestock exposed to spoiled feed (Wesley 1999), adaptation to their new environment commences with the activation of a set of virulence-associated stress mediators (Vazquez-Boland et al. 2001). This can lead to udder invasion in mammals, resulting in mastitic milk (Bourry and Poutrel 1996), and fecal shedding for further transmission (Nightingale et al. 2004). Though 
cattle tend to have a higher risk of exposure via contaminated silage, poultry products are typically more contaminated than beef, likely due to greater carcass cross-contamination in poultry processing plants (Richmond 1990).

The environmental robustness inherent in soil microbes may help L. monocytogenes survive foodprocessing technologies such as salting, acidification, and refrigeration of contaminated meat and dairy products (Lammerding and Doyle 1990). As seen analogously with other zoonotic pathogens, there is evidence that compared to Listeria strains found in the environment, those adapted to growth in animals are more often associated with large-scale human outbreaks (Graves et al. 2005).

\section{EXPEC}

Though E. coli O157: $\mathrm{H} 7$ remains the leading cause of acute kidney failure in previously healthy U.S. children (Thorpe 2004), fewer than 75,000 Americans become infected every year and fewer than 75 die, at a cost to the U.S. healthcare system of less than 500 million USD (CDC 2005a). An estimated 150 million Americans contract E. coli urinary tract infections (Stamm and Norrby 2001), however, resulting in an estimated 36,000 dying from sepsis at an annual cost in the billions (Russo and Johnson 2003).

Urinary tract infections (UTIs) are the most common bacterial infections in women of all ages (Beers and Berkow 2005) and among the most common bacterial infections around the world overall (Foxman 2002). From a physician's perspective, UTIs are increasingly harder to treat as antibiotic resistance among the chief pathogen, uropathogenic E. coli (UPEC), becomes more common (CDC 2005c). It is generally agreed that the immediate UPECsource causing human UTIs is an individual's owncolonic flora (Johnson and Stell 2000), but mystery has surrounded how these virulent clones come to inhabit the colon in the first place. A recent hypothesis posits that most UTIs may be of zoonotic origin (Rodriguez-Siek et al. 2005).

Pathogenic strains of $E$. coli have long been recognized as sources of foodborne diarrhea (Smith et al. 2007). Strains that cause disease outside the intestine are known as extraintestinal pathogenic E. coli (ExPEC) and include UTI-causing uropathogenic E. coli (UPEC), newborn meningitis-causing E. coli (NMEC), and avian pathogenic E. coli (APEC). The zoonotic hypothesis suggests that retail poultry harboring APEC represents a foodborne source of $E$. coli clones capable of causing human UTIs (UPEC) and possibly newborn meningitis as well (Rodriguez-Siek et al. 2005).

A prospective two-year market survey of 1,648 diverse food items from ten retail markets in the Minneapolis-St. Paul area detected the presence of $E$. coli in $69 \%$ of the pork and beef tested and $92 \%$ of the poultry samples, consistent with the contamination of carcasses with fecal flora during slaughter and processing. Half of the poultry samples were contaminated with extraintestinal pathogenic $E$. coli bacteria, supporting the notion that human UTI-type E. coli may be a foodborne pathogen. Interestingly, significantly less $E$. coli contamination was found on meat samples purchased from natural food stores (Johnson et al. 2005).

A number of studies have since indicated a commonality of virulence factors in human and animal strains of ExPEC (Smith et al. 2007). Moulin-Schouleur et al. (2007) used PCR-based phylotyping and multilocus sequence typing to determine the phylogenetic relationships between 39 APEC strains and 51 human EXPEC strains. The research team also compared the virulence genotype and pathogenicity of APEC strains and human ExPEC in laboratory chicks. They concluded that APEC strains belong to the same highly pathogenic clonal group as E. coli strains of the same serotypes isolated from human cases of neonatal meningitis, urinary tract infections, and septicemia.

Johnson et al. (2007) were the first to fully sequence an APEC isolate and found compelling genomic similarity between it and several human ExPEC strains. Based on a screening of a large collection of APEC, UPEC, and NMEC strains for virulence features, O-types, and phylogenetic background, Ewers et al. (2007) speculated that not only may poultry be a vehicle or even reservoir for human ExPEC strains, but may also serve as an armory of virulence-associated genes that may transform commensal human gut $E$ coli into extraintestinal UPEC and NMECstrains. Another recent report found that APEC plasmids 
can contribute to the urovirulence of $E$. coli in mammalian hosts, supporting this line of reasoning (Skyberg et al. 2006).

These studies bolster the hypothesis that at least some APEC strains are a source of UPEC, indicating that these organisms potentially represent a new class of foodborne zoonotic pathogens (Smith et al. 2007). Validation of this hypothesis will require assessment of the ability of APEC to persist on retail poultry, traverse the human intestinal tract, and ascend and colonize the human urinary tract. Epidemiological studies will also be required to confirm that such a phenomenon actually occurs (Johnson et al. 2007). A broader geographic sampling of poultry products would also be useful (Smith et al. 2007).

In chickens, APEC causes colibacillosis, now one of the most significant and widespread infectious diseases in the poultry industry worldwide (Barnes et al. 2003). In a prospective study of broiler chicken operations in Canada ranging in size from 7,700 to more than 100,000 birds, the risk of pathogenic E. coli infection was significantly associated with both increased farm size and chicken stocking density (Elfadil et al. 1996). In caged egg-laying hens, the most significant risk factor for flock infection was hen density per cage. The less average space each bird had, the higher the risk of outbreak. Vandekerchove et al. (2004) calculated that every additional liter of cage living space afforded to each hen would be associated with a corresponding 33\% drop in the risk of colibacillosis outbreak and suggested that new European laws coming into effect implementing more spacious cages would have a beneficial effect on the layers' health. If the zoonotic origin of UPEC is established, such laws may have beneficial effects on consumers as well.

\section{Multidrug-Resistant Bacteria}

The emergence and dissemination of multidrug-resistant zoonotic bacterial pathogens are increasingly being recognized as a public health problem of global significance (Moore et al. 2006). The use of growthpromoting antibiotics in industrial animal agriculture may be responsible for the majority of the increases in antibiotic-resistant human isolates reported (Tollefson et al. 1999).

The discovery that antibiotics could expedite growth in chickens was announced at the annual meeting of the American Chemical Society in 1950. By 1951, the U.S. Food and Drug Administration (FDA) approved the addition of penicillin and tetracycline to chicken feed as growth promoters, setting the stage for pharmaceutical companies to mass-produce antibiotics for animal agriculture (Boyd 2001).

The majority of present-day global antibiotic production is used in animal agriculture (Tilman et al. 2002). Mellon et al. (2001) estimate that up to $70 \%$ of antimicrobials used in the United States are used as feed additives for chickens, pigs, and cattle for non-therapeutic purposes. Additionally, three antibiotics have been approved for use in the U.S. aquaculture industry, which is estimated to consume in excess of 20,000 kilograms of antibiotics annually. Given the scale and intensification of the broiler industry, though, chickens raised for meat have historically consumed the largest share (Viola and DeVincent 2006).

By the 1970s, nearly all commercially raised poultry in the United States were reared on antibioticcontaining feed. By the end of the 1990s, U.S. poultry producers may have been using more than 4.5 million kilograms of antibiotics a year, a $300 \%$ increase from the 1980 s on a per-bird basis (Mellon et al. 2001). The proportion of antibiotics used in livestock production for growth promotion rather than therapeutic purposes may exceed $90 \%$ (Anderson et al. 2003).

Why the low-level feeding of antibiotics promotes faster weight gain in animals raised for meat is not completely understood (Graham et al. 2007). Germ-free chicks raised in germ-free environments seem to grow faster than chickens in unsanitary environments, suggesting that immune activity may divert energy from maximal growth (Klasing et al. 1987). Indeed, germ-free chicks administered antibiotics experience no change in growth rates, whereas commercially raised chickens demonstrate a remarkable spurt in growth (Freeman et al. 1975). In the relatively unsanitary conditions of intensive animal husbandry, normal physiological processes such as growth may be impaired in light of the infectious load to which animals are exposed, which may be mitigated by a constant influx of antibiotics (OTA 1979). 
Although the use of antibiotics in livestock production can lead to an improvement in feed efficiency and carcass quality (Andreasen et al. 2005), the indiscriminate use of antibiotics may select for drug-resistant zoonotic pathogens. Antibiotics and antibiotic-resistant bacteria have been detected in the air, groundwater, and soil around farms, and on retail meat (Smith et al. 2005). In the Minneapolis-St. Paul ExPEC market survey, for example, more than $80 \%$ of the $E$. coli recovered from beef, pork, and poultry products were resistant to one or more antibiotics, and greater than half of the isolates from poultry were resistant to more than five drugs (Johnson et al. 2005). Humans may then be exposed to these pathogens through infected meat, vegetables fertilized with raw manure, or water supplies contaminated by farm animal waste (Acar and Moulin 2006).

At least 17 classes of antimicrobials are approved for farm animal growth promotion in the United States, including $\beta$-lactam, tetracycline, aminoglycoside, and macrolide classes, all of which are important for clinical medicine (Anderson et al. 2003). Indications of adverse human health implications have been derived from multiple lines of evidence: epidemiological studies tracing drug-resistant human infections to specific farm animal production facilities, timelines showing antibiotic-resistant infections in farm animal populations preceding the emergence of the same resistance in humans, and microbial studies showing that antibiotic-resistant zoonotic bacteria may transfer that resistance to commensal human gut bacteria (WHO/FAO/OIE 2003). Garofalo et al. (2007) recently demonstrated that antibiotic-resistance genes could be detected directly in chicken meat and pork. The strongest evidence may be data from the European Union's experience, which showed that after antibiotics of human importance were banned for growth promotion in 1998, there was a subsequent decrease in the levels of antibiotic-resistant bacteria in farm animals, on meat, and within the general human population (Smith et al. 2005). Recently a large poultry corporation announced a reduction in antibiotic use in feed (Tyson Foods 2007), and, in 2005, the quinolone enrofloxacin was withdrawn from the U.S. market (FDA 2005).

4a. Campylobacter. Campylobacter species have long been associated with livestock diseases, but only since the 1970s have they been recognized as significant zoonotic poultry pathogens (Moore and Matsuda 2002). Campylobacter spp. are currently one of the leading causes of bacterial gastroenteritis in the world, resulting in an estimated 1.4 million infections, 13,000 hospitalizations, and 100 deaths annually in the United States alone (Friedman et al. 2000). The role of poultry in human Campylobacteriosis was clearly demonstrated in Belgium in 1999 when the discovery of high dioxin levels in feed led to a temporary deficit in the retail sale of poultry and eggs. This resulted in an estimated $40 \%$ reduction in the numbers of reported human Campylobacter cases, which rose back to baseline when normal poultry consumption levels resumed (Tenover et al. 2002). A similar phenomenon was reportedly recorded in the Netherlands in 2003 when avian influenza led to the depopulation of 30 million birds (Rosenquist et al. 2004). Campylobacter colonization of chickens is typically asymptomatic in the birds themselves but can lead to significant fecal shedding (Doyle and Erickson 2006). A single, contaminated drop of raw retail chicken "juice" may be enough of an infectious dose (McNamara 2002), and infectivity may persist on a cutting board-type surface for hours (Cools et al. 2005).

Typically, Campylobacteriosis is a self-limited, diarrheal illness only requiring fluid replenishment, but antimicrobial treatment may be prudent for patients with severe, prolonged, or systemic infections. If bacteremia is suspected, a fluoroquinolone antibiotic such as ciprofloxacin is commonly prescribed (Peterson 1994). Fluoroquinolone antibiotics have been used in human medicine since 1986, but widespread fluoroquinolone-resistant Campylobacter did not arise in the United States until after quinolones such as enrofloxacin were licensed for use in chickens in 1995 and 1996 for poultry housewide administration in drinking water (Gupta et al. 2004). In countries such as Australia, which reserved fluoroquinolones exclusively for human use, resistant Campylobacter have remained rare (Price et al. 2005).

The FDA developed a quantitative risk assessment model that suggested the consumption of an estimated 1.2 billion pounds of boneless chicken contaminated with fluoroquinolone-resistant Campylobacter in the United States in 1999 led to 153,580 human infections and a delay in effective treatment in 9,261 Americans (FDA 2001). Helms et al. (2005) showed that such a delay may lead to more complications and higher risk of adverse outcome. 
A lengthy legal process to stop the use of enrofloxacin in poultry culminated in its withdrawal from the market, effective September 2005 (Nelson et al. 2007). As the United States is the world's largest producer and exporter of poultry meat (FAO 2007b), this decision is expected to have a significant impact, though fluoroquinolone-resistant Campylobacter spp. are expected to continue to circulate in poultry flocks for some time (Humphrey et al. 2005). Meanwhile, U.S. Campylobacter isolate resistance to erythromycin continues to climb, and, in 2005, the first multidrug-resistant isolate was detected, $C$. jejuni resistant to ciprofloxacin, erythromycin, and ceftriaxone (Moore et al. 2006).

4b. Salmonella. The vast majority of foodborne Campylobacter infections involve an acute, self-limited, diarrheal episode, but have been linked to the development of a rare intestinal lymphoma (Lecuit et al. 2004) and are now the suspected cause of up to $25 \%$ of U.S. cases of irritable bowel syndrome (Samuel et al. 2004). Approximately 1 of 1,000 Campylobacter infections results in Guillain-Barr'e syndrome, a paralytic neurological complication (Peterson 1994). With the virtual elimination of polio in the developed world, this zoonotic poultry pathogen is now the most common cause of acute flaccid paralysis in developed countries (Tauxe 2002). Similarly, up to $15 \%$ of those suffering from Salmonella infection may develop a chronic condition of joint inflammation. An estimated 100,000 to 200,000 Americans suffer from arthritis arising directly from foodborne infections every year (McDowell and McElvaine 1997).

Annually in the United States, nontyphoidal Salmonella infections kill hundreds, hospitalize thousands, and may sicken more than a million (Mead et al. 1999). Salmonella infections are often also self-limited, but may progress to bacteremia in $3-10 \%$ of culture-confirmed cases, particularly in infants (Stutman 1994), for which antimicrobial therapy can be life-saving (Tauxe 1991). In adults, fluoroquinolones such as ciprofloxacin are the antibiotic of choice, while extended-spectrum cephalosporins such as ceftriaxone are considered preferred treatments for children (Fey et al. 2000). Growing Salmonella resistance to both frontline therapies has elicited global concern.

Multidrug-resistant Salmonella enterica serovar Typhimurium phage type 104 (DT104), which is resistant to up to six common antimicrobials including ciprofloxacin, rapidly emerged in the 1980 s as a global health problem. DT104 was first detected in humans and cattle but has since become common in poultry, particularly turkeys, as well as pigs and sheep (Threlfall 2000). Contact with sick farm animals and the consumption of chicken, pork sausages, and meat paste were early identified risk factors for DT104 infection, elevating the urgency of debate over the clinical consequences of drug resistance in zoonotic bacteria (Wall et al. 1994). Once acquired, the cluster of genes coding for multidrug resistance may be passed horizontally to other serovars, as may have been documented in poultry in Belgium (Cloeckaert et al. 2000). Comparing outbreaks caused by resistant versus pansusceptible Salmonella isolates, elevated morbidity (hospitalization) and mortality rates illustrate the clinical consequences of antimicrobial resistance (Holmberg et al. 1984).

The emergence and worldwide dissemination of DT104 has been speculatively blamed on antimicrobial use in aquaculture. DT104chloramphenicol resistance is due to floR, the florfenicol-resistance gene first identified in a piscine bacterium (Bolton et al. 1999) and presumably selected for by the use of florfenicolin aquaculture operations in Asia (Sano 1998). DT104 tetracycline resistance is likewise caused by a gene first identified in a fish pathogen (Zhao and Aoki 1992), and the DNA sequence encompassing both resistance genes on the DT104 chromosome is closely related to a plasmid first isolated in a third bacterial fish species in aquaculture operations in Japan (Kim and Aoki 1993). Furushita et al. (2003) showed that tetracycline-resistance genes found in hatchery tanks at fish farming operations and clinical (hospital effluent) bacterial isolates shared $92-100 \%$ sequence identity, and that resistance was experimentally transferable, suggesting that the human (hospital) and aquaculture compartments of the global environment behave interactively. The resistance determinants of DT104 may therefore have emerged among aquaculture bacteria and been horizontally transferred to DT104 (Angulo and Griffin 2000).

Although farmed fish may serve as a vehicle for the transmission of resistance-coding plasmids to commensal or pathogenic human bacteria (Rhodes et al. 2000), human-to-human transmission is so inefficient in industrialized countries (Cohen and Tauxe 1986) that the rapid global dissemination of these resistance gene clusters in the 1980s must be explained by other means. In the 1980s in Japan, where 
the use of florfenicol, tetracyclines, and 20 other antimicrobials are permitted in aquaculture production (Sano 1998), exports of fish meal rose over 500\%, reaching 223,859 tons in 1989 (FAO 2007b). Combined with the model presented by the international spread of a previous Salmonella serotype traced to the global distribution of contaminated fish meal with subsequent livestock and human isolate recovery (Clark et al. 1973), DT104 resistance gene clusters may have been distributed worldwide in the 1980s via contaminated livestock feed made out of farmed fish (Angulo and Griffin 2000). Perhaps because of host differences in thermoregulation (Alderman and Hastings 1998), aquatic zoonotics may be relatively rare, but antimicrobial resistance acquired by farmed fish pathogens may horizontally transfer to pathogens of commonly eaten homeotherms (Kruse and Sorum 1994).

Once regional distribution is achieved, the persistence and spread of resistance gene clusters could be facilitated by the recycling of livestock waste into feed (Turnbull 1979) and further antimicrobial use. Preweaned U.S. dairy calves, for example, are suckled with tetracycline-containing milk replacer, which is commonly used commercially to prevent diarrhea thought exacerbated by maternal colostrum deprivation (Berge et al. 2005). Berge et al. (2006) conducted a field trial that demonstrated that the use of neomycin/tetracycline-medicated milk replacer in preweaned calves selected for bacteria with resistance clusters to antimicrobials not used in the study or on the ranch.

Concern over the promiscuity of self-transmissible plasmids coding multidrug-resistance reached new levels with the detection of a multidrug-resistant strain of Yersinia pestis (IP275) in 1995 (Galimand et al. 1997). The etiological agent of plague, Y. pestis, is a zoonotic bacterial pathogen that has caused multiple pandemics resulting in an estimated 200 million human deaths but, with antibiotic treatment and prophylaxis, has been effectively controlled throughout most of the world (Perry et al. 1997). Y. pestis IP275 contains a self-transmissible plasmid conferring high-level resistance to at least eight antimicrobials, including streptomycin, tetracycline, chloramphenicol and sulfonamides, drugs that are recommended for use in plague (Inglesby et al. 2000). ComparativeDNAanalysis of the Y. pestis IP275 plasmid showed a near identical backbone to multidrug resistance plasmids isolated from a Salmonella spp. and a fish pathogen, Yersinia ruckeri. Furthermore, Y. pestis IP275-like plasmids were isolated from beef, chicken, turkey, and pork meat samples over a wide geographic distribution, suggesting that the agricultural use of antibiotics may have broader biomedical, public health, and biodefense implications than previously recognized (Welch et al. 2007).

The most dramatic emergence of multidrug-resistant Salmonella over the last two decades may have been the appearance and global spread of Salmonella resistant to extended-spectrum cephalosporins such as ceftriaxone, the third-generation cephalosporin used to treat invasive blood infections in children (Arlet et al. 2006). In addition to its resistance to five drugs typically found in DT104-ampicillin, chloramphenicol, streptomycin, sulfamethoxazole, and tetracycline-Salmonella enterica serotype Newport isolates resistant to at least nine antimicrobials (S. Newport MDR-AmpC) have arisen, resistant to amoxicillin/clavulanate, cefoxitin, ceftiofur, and cephalothin as well, with decreased susceptibility to ceftriaxone (MIC, $16 \mu \mathrm{g} / \mathrm{ml}$ ) (Harbottle et al. 2006).

Salmonella serotype Newport has emerged as the third most common serotype causing human salmonellosis in the United States, with a doubling of laboratory-confirmed cases between 1997 and 2001. Human outbreaks have been linked to the consumption of hamburger (Spika et al. 1987), pork (Narain and Lofgren 1989), chicken (Anand et al. 1980), and fish (Heinitz et al. 2000). The increase in S. Newport isolates is likely associated with the rapid dissemination of multidrug-resistant strains (CDC 2002). By 2004, Salmonella resistance to extended-spectrum cephalosporins was identified in 43 countries (Arlet et al. 2006).

The first documented domestically-acquired U.S. case of Salmonella infection resistant to ceftriaxone (along with 12 other antimicrobials) was isolated in 1998 and found closely matched to isolates in a local outbreak of bovine salmonellosis, providing additional evidence that antibiotic-resistant strains of Salmonella evolve primarily in livestock (Fey et al. 2000). In 1998, 2\% of Salmonella isolates collected from chickens and cattle in sampled U.S. slaughter plants were resistant to ceftriaxone (FDA/USDA/CDC 1998). 
The use of ceftiofur in farm animals has come under increasing scrutiny as a selective factor responsible for the emergence and persistence of ceftriaxone-resistant enteric pathogens such as S. Newport MDRAmpC. Ceftiofur was the only cephalosporin approved for systemic use in pigs, sheep, chickens, and turkeys in the United States, and cross-resistance between ceftiofur and ceftriaxone (and cephamycins) has been demonstrated. In thisway, the widespread use of ceftiofur in animals raised for human consumption may have contributed to ceftriaxone resistance in Salmonella, whichmaythen be transmitted to consumers through the food supply (Dunne et al. 2000). Controversially, a fourth-generation cephalosporin, cefquinome, is currently under review for FDA approval for use in cattle (Feldgarden 2007).

Animals bearing Salmonella and Campylobacter can remain healthy carriers with horizontal transmission mediated by fecal shedding. Although Campylobacter colonization of the avian reproductive tract may lead to external egg contamination (Byrd et al. 2007), Salmonella can lead to ovarian infection, resulting in vertical transmission and the potential for additional zoonotic risk (Gast and Beard 1990).

The leading cause of salmonellosis in the world is now the egg-borne serovar Salmonella enterica enteritidis (S. enteritidis) (Baumler et al. 2000).With beginnings traced to the mid-1970s, by the 1990s human S. enteritidis infections had reached pandemic proportions (Pang et al. 1995). In the United States, S. enteritidis cases began to appear in the Northeast in the late 1970s and spread to the MidAtlantic States by the mid-1980s (Braden 2006). A single U.S. outbreak in 1994, in which tanker trailers previously carrying raw eggs contaminated the premix of a nationally distributed ice cream, resulted in an estimated 224,000 human cases of $S$. enteritidis infection (Hennessy et al. 1996). On average, though, the CDC estimates that 50,000 to $110,000 \mathrm{~S}$. enteritidis infections each year in the United States can be attributed to eggs (Braden 2006).

In S. enteritidis outbreaks in which a food source was confirmed, $75 \%$ were traced to eggs or eggcontaining products (Braden 2006). Eggs are one of the few animal products that are frequently eaten raw or undercooked, as in such items as homemade or restaurant-produced salad dressings, hollandaise sauce, mayonnaise, ice creams, or beverages including eggnog. Humphrey et al. (1989) showed that "sunny-side up" frying, for example, was insufficient to destroy S. enteritidis inoculated into yolks at concentrations typical of transovarian infection.

S. enteritidis infections have fallen over the last decade in the United States due largely to farm-based assurance programs, early and sustained egg refrigeration, and educational efforts targeted at food service workers and consumers (Braden 2006), but these domestic efforts and successes may pale in comparison to those made in other countries (Hopp et al. 1999). Though the poultry industry operates under severe cost pressures, changes in management practices may be necessary to make significant further progress in reducing this zoonotic risk (Humphrey 2006).

Holt et al. (1998), for example, showed that poor ventilation and high dust levels appear to aid dissemination of $S$. enteritidis among chickens via colonization of exposed mucosal surfaces, suggesting that better poultry house design to improve air quality may decrease $S$. enteritidis infection rates. The high density of modern commercial chicken populations may not only increase risk due to the number of potentially infectious contacts and the level of airborne particulates and litter contamination (Braden 2006), but may have physiological effects that facilitate infection mediated through the sympatheticadrenal medullary axis. A range of food production-relevant stresses have been shown to facilitate extraintestinal invasion by bacterial pathogens (Swildens et al. 2004).

Stress caused by severe overcrowding, for example, such as in high-density layer battery caging (Humphrey 2006), has been shown to increase Salmonella infection susceptibility in mice (Kuriyama et al. 1996). The physiologic stress associated with the forced molting of commercial egg-laying flocks to extend their productive life increases S. enteritidis shedding (Holt et al. 1994) and may double the incidence of egg contamination (USDA 1998). Noradrenaline (NA) is a catecholamine released by animals under stress. Chickens who are acutely (Knowles and Broom 1993) and chronically (Cheng et al. 2002) stressed may have higher intestinal and circulating levels of NA. Noradrenaline is taken up by $E$. coli, increases bacterial growth rates (Kinney et al. 2000), and may up-regulate certain virulence factors 
(Lyte 2004). In iron-restricted media used to simulate the intestinal mucosal surface, NA markedly increases Campylobacter growth rates and may increase bacterial motility, colonization, and the expression of virulence factors (Humphrey 2006). This may contribute to the phenomenon of $C$. jenuni isolation from deep muscle tissues in retail poultry (Humphrey 1991), which may present a greater public health risk than surface contamination. Stress-induced neurotransmitters may also increase the growth rates of Salmonella spp. (Bailey et al. 1999), Listeria, (Coulanges et al. 1998), and other zoonotic pathogens (Belay et al. 2003), as well as increase the expression of virulence factors in Pseudomonas aeruginosa (Kinney et al. 2000).

\section{Bovine Spongiform Encephalopathy}

Certain modern agricultural practices to enhance productivity may have unintended public health consequences (Phua and Lee 2005). The use of antimicrobials for growth promotion is one such practice, and the continued feeding of slaughterhouse waste, blood products, and excrement to livestock may be another (Sapkota et al. 2007).

Annually, the United States produces the majority (NRA 2005) of the estimated 10 million tons of animal protein concentrates incorporated worldwide into livestock feed, such as meat, blood, and bone meal (WHO and OIE 1999). Incomplete inactivation of pathogens during processing may pose a risk of disease transmission. U.S. livestock feeds incorporating meat byproducts in particular (McChesney et al. 1995) have been found to be contaminated with a number of zoonotic pathogens such as Salmonella spp. (Crump et al. 2002) and E. coli O157:H7 (Dargatz et al. 2005). Testing 165 rendered animal protein products originating from poultry, cattle, and fish at a poultry feed mill, Hofacre et al. (2001) found antibiotic-resistant bacteria in $85 \%$ of samples.

Bovine spongiform encephalopathy (BSE) may have arisen because of the inclusion of the rendered remains of sheep infected with an ovine spongiform encephalopathy in cattle feed, suggesting that animal-based feed additives may play a role in zoonotic disease emergence as well (Wilesmith et al. 1991). The further recycling of the remains of infected cattle into cattle feed likely contributed to the spread of the epizootic in the United Kingdom, where BSE was first recognized in 1985 (Anderson et al. 1996), and its subsequent spread to 24 countries (USDA APHIS 2005a).

BSE belongs to a class of transmissible spongiform encephalopathies thought caused by prions, an unconventional class of proteinaceous pathogens uniquely resilient to food preparation safeguards, demonstrating limited survival even after incineration at $360 \pm C$. Exceptional prion resistance to heat, formalin (Pattison 1965), and radiation (Gibbs et al. 1978) presumably derive from the putative lack of associated nucleic acid (Prusiner 1998).

A British Medical Journal editorial described BSE as resulting from an "accidental experiment on the dietary transmissibility of prion disease between sheep and cows" (Harrison and Roberts 1992). A subsequent "accidental experiment"-with humans-may have started in the late 1980s, as tissues from infected cattle increasingly entered the human food supply (Phillips et al. 2000). Approximately 100 human cases of variant Creutzfeldt-Jakob disease (vCJD), the invariably fatal neurodegenerative disease (Collinge 1999) thought caused by the consumption of contaminated bovine products (Bruce et al. 1997), have been neuropathologically confirmed in the United Kingdom (NN 2007b). The potentially decadeslong incubation period (Brown et al. 2000), combined with the discovery of an infection-positive human appendectomy tissue sample removed during routine surgery (Hilton et al. 2002), have raised concerns about the possibility of secondary iatrogenic transmission via blood products or surgical instruments (Zobeley et al. 1999). The fourth case of probable transfusion transmission of vCJD infection in the United Kingdom was recently reported (NN 2007a).

Experimental verification of blood infectivity (Houston et al. 2000) has renewed criticism over the practice of weaning U.S. dairy calves on milk replacer containing bovine blood products (DG-SANCO 2002), given the presence of BSE in North America (USDA 2006). In 1997, the U.S. FDA restricted the use of tissues considered particularly risky—bovine skull, brain, eyes, parts of the vertebral column, spinal cord, trigeminal and dorsal root ganglia, tonsils, and distal ileum-in the formulation of cattle and other 
ruminant feed, but continued to allow the intraspecies recycling of ruminant blood (GAO 2002). Most newborn calves in the United States are separated from their dams within 12 hours, many immediately after birth, so the milk can be marketed for human consumption. Though some dairy producers still wean calves on whole milk, the majority reportedly use milk replacer, which may contain spray-dried cattle blood as a cheap source of protein (USDA APHIS 1993).

The bovine neurological and lymphoid tissues specified as higher risk may not be fed legally to ruminants but are still allowed to be incorporated into nonruminant feeds and subsequent waste products are then permitted in ruminant feeds (USDA FSIS 2005). The use of ruminant meat and bone meal in the diets of broiler chickens, for example, may pose the risk of cycling prions back to cattle if the broiler litter, which may contain spilled feed and poultry waste, is then fed back to cattle (Rankins et al. 2002). Although excrement from other species is fed to cattle as well (Haapapuro et al. 1997), poultry waste is considered more nutritious for cows than swine waste or cattle dung (Fontenot et al. 1971). As much as eight times cheaper than foodstuffs like alfalfa (Fontenot 2001), poultry litter has been used as an economical feedstuff in the United States in place of hay (Rude and Rankins 1997) since the 1950s (Noland et al. 1955).

Two human outbreaks of Salmonella infection tied to the use of meat and bone meal in feed were recorded in the United Kingdom decades before the emergence of BSE (Knox et al. 1963; Pennington et al. 1968). Although the U.K. rendering industry may not have been able to foresee the full extent of the potential hazard of continuing this practice, the U.S. rendering industry has no such justification. In general, the livestock industries have reportedly tended to favor stop-gap measures such as food irradiation rather than fundamental changes in farm management practices (Nestle 2003). Investments in chemical carcass dehairing technologies to lessen fecal contamination in cattle processing plants (Nou et al. 2003) and the investigation of bacteriophage application to fresh poultry (Doyle and Erickson 2006) are examples of this trend.

One reason poultry products may be such common carriers of foodborne diseases (Gregory et al. 1997) is that unlike the potentially manure-encrusted hides of cattle, for example, the skin can be eaten with the meat. Efforts to improve on-farm hygiene to decrease eventual carcass surface contamination may be more effective in the long run than recently proposed technologies such as employing equipment designed to exert abdominal pressure to force out cloacal contents before scalding (Wagenaar et al. 2006) and the proposed use of either mechanical plug, stapling, or cyanoacrylate adhesive technology to seal the rectal cavity of farmed birds during processing (NN 1995).

The BSE crisis in the United Kingdom affected major institutional reform in government infrastructure, leading to the establishment of the Food Standards Agency independent from the perceived bias towards producer interests in the Ministry of Agriculture, Fisheries, and Food (Wales et al. 2006). The U.S. congressional Government Accountability Office (GAO 2001) and Institute of Medicine (IOM 1988) have called for the similar formation of a U.S. food safety authority independent of the USDA in hopes of further reducing the risk of foodborne zoonotic infections.

\section{B. Streptococcus Suis}

China is the world's largest producer of pork (FAO 2007b), scaling up the size of commercial operations (Bean and Jianping 2005) and doubling production to more than 50 million tons between 1990 and 2005, the year the largest pork-producing province (Xin et al. 2001) suffered an unprecedented human outbreak of the emerging zoonotic pig pathogen Streptococcus suis (Lun et al. 2007). S. suis is a common cause of meningitis in intensively farmed pigs worldwide (Sanford and Higgins 1992) and, similarly, presents most often as purulent meningitis in humans who handle or butcher sick pigs (Huang et al. 2005). Thought due to inner ear involvement (Kay 1991), 39\% of human survivors may suffer permanent sensorineural hearing loss (Wang et al. 2007).

In summer 2005 in Sichuan, China, a total of 215 probable cases of human S. suis infection were reported-more than the total number of previously recorded human cases worldwide (Tang et al. 2006) - following a local epizootic involving approximately 80,000 pigs (Lun et al. 2007). Fatal outcomes 
were reported in $39(18 \%)$ of human cases (Yu et al. 2006), perhaps triple the case fatality rate seen previously in sporadic human cases elsewhere (Normile 2005). Though the increased mortality in China may reflect regional differences in treatment access (Sriskandan and Slater 2006), most deaths appeared due to a potentially novel form of invasive toxic shock syndrome, combining deep-tissue infection and vascular collapse (Tang et al. 2006). In cases of S. suis septic shock, mortality may exceed $70 \%$ irrespective of treatment (Lun et al. 2007).

The first human case of S. suis infection was reported in Denmark in 1968 (Arends and Zanen 1988). Cases have since been reported in many countries with intensive swine production (Lun et al. 2007) in which S. suis has emerged, particularly during the past 15 years (Gottschalk and Segura 2000), as a major cause of porcine infection and economic loss (Escudero et al. 2007). In the United States, the world's second-largest pork producer (FAO 2007b), S. suis may cost the swine industry more than 300 million USD annually (Staats et al. 1997). Though human cases of S. suis meningitis may likely be underdiagnosed and misidentified (Gottschalk 2004) due to the lack of adequate surveillance (Cole et al. 2000), the first U.S. case was reported in 2006 (Willenburg et al. 2006). The American Association of Swine Veterinarians implicates increasing swine industry intensification as a critical factor in the emergence of porcine zoonoses in general, via both elevated environmental pathogen loads and the impairment of porcine host immunity (Meredith 2004). S. suis may fit this pattern (USDA APHIS 2005b).

S. suis is enzootic in most countries with intensively managed pig populations with high rates of asymptomatic carriers documented. Clinically normal pigs may harbor $S$. suis in their nasal cavity, palantine tonsils, and genital and alimentary tracts, and play an important role in the propagation of $S$. suis via "nose-to-nose" oronasal transmission (Arends et al. 1984). Isolating S. suis from tonsillar crypts at slaughter, Clifton-Hadley (1986) reported herd carrier rates as high as $100 \%$.

S. suis is likely transferred from vaginal secretions to the oral cavity of the piglet during parturition and colonizes the tonsils soon after birth (Amass et al. 1996). While experimental intravenous inoculation results in high morbidity and mortality (Busque et al. 1997), intranasal inoculation, the presumed natural route, often results in no or limited disease, lesions, and mortality (Iglesias and Trujano 1992). S. suis can become invasive, though, and result in a wide range of diseases in pigs (Staats et al. 1997). Pneumonia has historically been the primary porcine presentation, but meningitis and endocarditis have become more common, attributed to changing housing conditions and management practices (Aarestrup et al. 1998).

The factors responsible for $S$. suis traversing the mucosal barrier to induce disease are not known at this time (Gottschalk and Segura 2000), but Pallares et al. (2003) showed that pre-irritation of the nasal cavity with $1 \%$ acetic acid an hour before intranasal inoculation could result in invasive meningitic disease. This suggested that mucosal damage, stress, or both, induced by the acid pretreatment, allows $S$. suis to become septicemic. Once invasive, S. suis may then be spread via respiratory droplets (Berthelot-Herault et al. 2001) or more directly via contact with contaminated blood on improperly sterilized castration scalpels, tooth-cutting pliers, or tail-docking knives (Du 2005). At $25 \pm C$, S. suis has been shown to survive for 24 hours in dust and for eight days in feces (Clifton-Hadley and Enright 1984).

A number of factors may have contributed to the scale of the 2005 outbreak in China, i.e., a (still putative) highly virulent $S$. suis clone, the eating of undercooked pork from sick pigs, and delayed diagnosis and treatment (Lun et al. 2007). Focus, however, has fallen on the way in which pigs were raised. Poor housing conditions, such as overcrowding, inadequate ventilation, and related physiological stress, were considered risk factors for the initial epizootic outbreak (WHO 2005).

China now has swine facilities confining more than 50,000 head (Simpson et al. 1999), which may have stocking densities and sanitation levels exceptionally conducive to the emergence and spread of this disease (Arends et al. 1984). Dee et al. (1993), for example, showed experimentally that overcrowding and social stress led to higher-than-average $S$. suis infection rates. Coinfection with porcine reproductive and respiratory syndrome virus (PRRSV) or Aujeszky's disease virus can exacerbate S. suis infection in pigs (Iglesias et al. 1992). PRRSV is typically enzootic in intensive pig production settings (Chung et al. 1997), and Aujeszky's disease, also on intensive pig farms (Chew-Lim and Ng 1987), shows a stress- 
induced susceptibility in pigs (de Groot et al. 2001) (but not mice) (de Groot et al. 1999), suggesting that the association between S. suis infection and high-density pig herds is likely multifactorial (Clifton-Hadley and Enright 1984).

A similar pattern is found in other porcine respiratory diseases, which have been linked to the increased crowding of pigs, both per pen and per building (Enøe et al. 2002). Based on the isolation of pathogens from air samples taken inside production facilities, Madec and Rose (2003) suggested diminished air volume per animal may increase the concentration of infectious particles and thereby facilitate aerosol spread. This seems to be the case with swine influenza risk, which has been associated in commercial pig operations with increased density of pigs per pen, pigs per operation, and pigs per municipality (Maes et al. 2000), though this may derive from a combination of pathogen density and confinement-related stressors.

Breeding sows restricted to narrow stalls, as is common during gestation and farrowing in intensive production, produce lower levels of antibodies in response to an experimental challenge (Siegel 1983). Measures as simple as providing straw bedding for pigs may improve immune function by eliminating the immunosuppressive stress of lying on bare concrete (Andr'e and Tuyttens 2005). Ewald et al. (1994), for example, found that straw bedding was linked to decreased risk of swine flu infection.

Although S. suis was isolated from 6\% of raw pork products sampled in Hong Kong in 2005 (Ip et al. 2007), data from reportedly all epidemiological surveys on outbreaks in China strongly indicate that direct contact with sick pigs is necessary for zoonotic infection (Lun et al. 2007). The infection rate of pig farmers, abattoir workers, meat-processing workers, and veterinarians has been estimated to be 1,500times higher than that of the general population (Arends and Zanen 1988). S. suis, therefore, remains essentially an occupational zoonosis. Especially given concern over rising emergence of fluoroquinoloneresistant strains now reported among clinical swine isolates (Escudero et al. 2007), the exclusion of splenectomized individuals from the pork trade has been suggested (Watkins et al. 2001).

\section{Avian and Pandemic Influenza}

The dozens of emerging zoonotic threats that have characterized this third era of human disease must be put into context. The total number of human cases reported of $S$. suis infection worldwide is approximately 400 (Tang et al. 2006). Nipah virus and BSE prions seem to have so far infected fewer still. SARS-CoV did infect thousands and HIV millions, but there may be only one known pathogen capable of rapidly infecting billions: influenzavirus $A$.

Influenza has been called the "last great plague of man" (Kaplan and Webster 1977). Unlike infectious diseases like malaria, for example, which tend to be confined equatorially, or HIVIAIDS, which is only fluid-borne, influenza may be the only pathogen carrying the potential to infect a substantial percentage of the world's population within a matter of months. In the 4,500 years influenza virus has been presumed to infect humans since the first domestication of wild birds (Shortridge 2003b), influenza has always been one of humanity's most contagious diseases (Mills et al. 2004). But only since 1997 with the recognition of the zoonotic potential of highly pathogenic avian influenza virus A subtype H5N1 has influenza also emerged as potentially one of the deadliest, with a reported case fatality rate (CFR) exceeding $60 \%$ (WHO 2007). Although H5N1 has resulted in more than 200 million birds killed or culled (FAO 2006b), the ratio of avian to human deaths exceeds a million to one, with only approximately 200 confirmed human deaths (WHO 2007).

Though infinitesimal compared to the 1.7 million human deaths attributed to TB every year (WHO 2006a) or the more than 3 million to AIDS (UNAIDS and WHO 2005), one reason the emergence of H5N1 has raised so much concern is that the last known time a wholly avian influenza virus resulted in human mortality, it triggered the single deadliest disease event in human history: the 1918-1919 influenza pandemic in which $30 \%$ of the world's population may have fallen ill (Burnet and Clark 1942) and 50 million may have died (Johnson and Mueller 2002). 
H5N1 is now almost exclusively a disease of birds (Yang et al. 2007). Should the virus have the dual capacity to acquire efficient human transmissibility while maintaining its human virulence, H5N1 (CFR »60\%) could theoretically trigger a pandemic that makes the 1918 pandemic (CFR »2.5\%) (Marks and Beatty 1976) seem in comparison like the seasonal flu (CFR »0.1\%) (Rosenau and Last 1980). This makes the factors underlying the continuous emergence of this zoonotic virus particularly important to understand.

\section{Avian Influenza}

1a. Shifting Ecology. Though first clearly described more than 800 years ago (reviewed in Potter 2001), influenza can be considered a perpetually emerging disease given its ability for antigenic transformation. Type $A$ viruses are subdivided into subtypes according to the antigenic nature of two surface proteins, hemagglutinin (HA) and neuraminidase (NA), of which there are $16(\mathrm{H} 1$ to $\mathrm{H} 16)$ and 9 (N1 to N9) known, respectively (Fouchier et al. 2005). "H5N1" denotes that the virus envelope displays the fifth hemagglutinin type in the WHO-naming scheme, along with the first neuraminidase type (WHO 1980a).

Laver and Webster (1972) were the first to suggest through ecological studies that aquatic birds are the natural reservoir for the virus, which, in wild ducks, tends to exist as an asymptomatic gastrointestinal infection (Webster et al. 1978). More recent recognition has been made of the possibility that shorebirds may also maintain part of the influenza gene pool (Sharp et al. 1993). In nature, then, the influenza virus has likely existed for millions of years as a harmless (Kida et al. 1980), enteric, waterborne infection of waterfowl (Markwell and Shortridge 1982).

Studies of ducks clustered on Canadian lakes show that up to $30 \%$ of juvenile birds may be actively shedding the virus (Hinshawet al. 1980b). Most infected ducks evidently only shed virus for a few days (Webster et al. 1993), but the presumed fecal-water-oral route of infection for aquatic birds (Markwell and Shortridge 1982) is thought efficient enough to have kept the virus circulating throughout the millennia (Shortridge 1992). The ducks excrete such high titers of virus that Hinshaw et al. (1979) were able to culture influenza viruses straight from unconcentrated lake water. Under permissive conditions, the virus is estimated to be able to persist for years in coldwater (Wobeser 1997) and possibly even survive in ice (Zhang et al. 2006). Given such high concentrations of virus, the efficiency of transmission (Webster et al. 1997), and with such environmental stability, influenzavirus A likely infects virtually all of the millions of ducks in the world sometime within their lives (Murphy 1993).

Human influenza pandemics are thought triggered by genetic infusion from this avian reservoir, resulting in influenzavirus A subtypes with surface proteins to which the present human generation is immunologically na"ive (Webster and Laver 1971). A purely avian H1N1 subtype is believed to have caused the 1918-1919 pandemic, presumably via adaptive mutation (Taubenberger et al. 2005), infecting an estimated one billion people over 12 months (Oxford et al. 2006). The "Asian flu" pandemic of 1957 and the 1968-1969 "Hong Kong flu" were generated when the circulating seasonal human subtype acquired newavian gene segments via reassortment (Scholtissek et al. 1978), each resulting in approximately one million deaths (Pyle 1986). One speculation as to why the 1918 pandemic virus may have cost as many as 25 times more human lives than both these pandemics combined (Johnson and Mueller 2002) is that as a wholly avian virus, no partial resistance may have existed in the human population (Cox and Subbarao 2000). H5N1, to which human vulnerability is universal, may be taking the same route of adaptation via a smoldering avian progenitor (Shortridge et al. 2000).

Apart from record human case fatality, $\mathrm{H} 5 \mathrm{~N} 1$ has exhibited other unique features unprecedented for avian influenza viruses: unpreadapted lethality to laboratory primates (Rimmelzwaan et al. 2003), ferrets (Govorkova et al. 2005), and rodents (Gubareva et al. 1998), with cases of strong neurovirulence (Maines et al. 2005); a mammalian host range including feline (Keawcharoen et al. 2004), canine (Butler 2006b), mustelid (Klopfleisch et al. 2007), and viverrid (Roberton et al. 2006) species; the establishment of distinct lineages all lethal for gallinaceous poultry, but not necessarily waterfowl (Sturm-Ramirez et al. 2005), and each with repeated transmission to humans (Smith et al. 2006); a seeming increase in thermal and, therefore, potential environmental stability (Swayne and Beck 2004; WHO 2006c); and geographic spread to the majority of countries in Eurasia (OIE 2007). The emergence of H5N1, however, is but one example 
of the myriad appreciable changes that seem to be taking place in the ecology and epidemiology of avian influenza (Webster et al. 2007).

Since the first recorded outbreak of H5 subtype highly pathogenic avian influenza (HPAI) occurred in 1959, 24 primary HPAI outbreaks have been reported. In the first 20 years of this 47-year interval, 1959 to 1978, four outbreaks were recorded, involving a combined total of less than 100,000 birds. In the next 20 years, 1979 to 1998, a tripling of the frequency of outbreaks was recorded with 13 outbreaks. The last seven years saw outbreaks of seven different HPAI viruses involving, on average, millions of birds each (Alexander 2007). With the exception of the 1983-1984 outbreak in Pennsylvania, which involved 17 million birds at an estimated cost exceeding 400 million USD (Lasley 1987), all of the first 12 of 24 outbreaks recorded since 1959 involved fewer than 500,000 birds, whereas 8 of the last 12 outbreaks involved more than 500,000 birds and 7 of the last 12 involved millions of birds. Even excluding H5N1, which has led to the deaths of hundreds of millions of birds across 44 countries and territories (OIE 2007), outbreaks in the last decade of HPAl viruses other than H5N1—namely H5N2, H7N1, H7N3, H7N4, and H7N7-have affected more than 50 million birds across five continents (Alexander 2007).

Historically, chickens have been considered a rare host for influenzavirus A. Prior to the 1990s, few influenza viruses were isolated from chickens and even fewer chicken-adapted lineages described (Liu et al. 2003). This pattern dramatically changed by the mid-1990s, as illustrated by the emergence, spread, and eventual establishment of a stable H9N2 lineage in domestic chickens throughout Eurasia (Xu et al. 2007).

Prior virologic survey studies made only 7 isolations of 3 subtypes of influenza viruses from 1,708 chickens sampled in a Hong Kong poultry processing plant between 1975 and 1979 (0.4\%), whereas 564 influenza viruses of 11 different HA subtypes were isolated in ducks (6.5\%) (Shortridge 1982). Chickens were considered such atypical hosts that although virologic surveillance in pigs in China continued, at the time human H5N1 infection was first recognized in 1997, the estimated 1,000 live poultry markets in Hong Kong were not under surveillance (Shortridge 1999).

In Hong Kong survey studies in the late-1970s, H9N2 viruses were isolated from waterfowl but not from chickens (Shortridge 1982). The earliest H9N2 isolate in a terrestrial bird was discovered in Hong Kong quail in 1992 (Guo et al. 2000). By 1995, H9N2 was causing outbreaks of severe respiratory disease in chickens in China (Guo et al. 2000) and Korea (Mo et al. 1997b) with mortality rates of 10-40\%. Currently, H9N2 is widely enzootic in chickens in Eurasia (Xu et al. 2007) and has infected children in Hong Kong SAR in 1999 (Lin et al. 2000) and 2003 (Butt et al. 2005).

The unprecedented isolation of other influenzavirus A subtypes in gallinaceous poultry has also been demonstrated recently. Since 1995, H6 and H3 viruses have been isolated from terrestrial poultry in China (Liu et al. 2003), Korea (Choi et al. 2005b), Taiwan (Lee et al. 2006), Italy (Campitelli et al. 2002), South Africa (Abolnik et al. 2007), and, in the United States, California (Webby et al. 2002), North Carolina, and Minnesota (Choi et al. 2004). Particular concern surrounds the reported increase in the prevalence in HongKongSARofH6N1viruses that possess seven of eight genes highly homologous to the H5N1 virus that triggered the 1997 human outbreak in Hong Kong (Chin et al. 2002). Surveillance data from 2000 to 2005 suggest multiple co-circulating $\mathrm{H} 6$ viruses have become established in gallinaceous poultry in southern China (Cheung et al. 2007).

Without more extensive pre-1990s surveillance data, it is not possible to accurately assess how much of this escalation is due to changes in detection or reporting, rather than actual changes in incidence. Given the prior data that are available on low pathogenicity avian influenza (LPAI) virus isolation (Shortridge 1982), as well as the often conspicuous nature of HPAI outbreaks (Capua and Alexander 2004), a true ecological shift seems to have occurred in avian influenza viruses in the 1990s.

1b. Shifting Epidemiology. Before 1997, known direct zoonotic infections with avian influenza viruses were considered rare and of little consequence (Subbarao and Katz 2000). The four isolated case reports that did exist all described detections of H7N7 viruses: the first from the blood of a patient with hepatitis in 1959 (Campbell et al. 1970), followed by three instances of conjunctivitis-a laboratory ocular splash in 
1977 (Taylor and Turner 1977), an animal handler sneezed on by an infected seal in 1981 (Webster et al. 1981), and a woman in England reporting a piece of straw entered her eye while she had been cleaning out her duck house in 1996 (Kurtz et al. 1996). There is no convincing evidence that human infections with H5 influenza viruses have ever occurred before H5N1 (Webster et al. 2007). The scarcity and largely benign nature of the human cases reported before 1997 are supported by studies showing that humans directly inoculated with avian influenza viruses from waterfowl, the influenzavirus A reservoir, show at most mild transitory infections in a minority of subjects (Beare and Webster 1991).

In contrast, since 1997, more than 300 reported cases of human H5N1 infection have been confirmed across a dozen countries, resulting in nearly 200 deaths, the slim majority of which were in children or young adults under the age of 20 (WHO 2007). Paralleling the ecological changes in a multiplicity of avian influenza subtypes since the 1990s, H5N1 is just one of five poultry influenzavirus A subtypes that have caused zoonotic infections since 1997: H9N2 infections in children in China in 1999 (Peiris et al. 1999) and 2003 (Butt et al. 2005); H7N2 infections in residents of New York in 2002 and Virginia in 2003 (CDC 2005b); H7N7 in poultry handlers and their family members in the Netherlands in 2003 (Fouchier et al. 2004); H7N3 infections in poultry workers in Canada in 2004 (Tweed et al. 2004) and likely Italy in 2003 (Puzelli et al. 2005); H10N7 infections in infants in Egypt in 2004 (PAHO 2004); and, in the United Kingdom, H7N3 in 2006 (Nguyen-Van-Tamet al. 2006) and H7N2 in 2007 (HPA 2007).

In 1993, children in Europe were reportedly infected with an H3N2 avian/human reassortment virus likely transferred from pigs (Claas et al. 1994). Combined with the unprecedented human/avian/porcine triple reassortment in North Carolina pigs in 1998 (Zhou et al. 1999) after a stable "classical" swine flu lineage had presided throughout the bulk of the century (Brown 2000b), this suggests that the ecology and epidemiology of swine influenza viruses have been changing since the 1990s as well.

Aside from the H5N1 panzootic, the avian influenza outbreak in the Netherlands in 2003 is likely the largest in history (Alexander 2007). Overshadowed by the burgeoning SARS epidemic at the time (Anderson et al. 2004), 30 million chickens were culled or killed (Du Ry van Beest Holle et al. 2005) and clinical infections were confirmed in 86 poultry handlers, including an attending veterinarian who later died in acute respiratory distress, and 3 of their family members (Fouchier et al. 2004). Human-to-human transmission was suspected due to the infections in household contacts, and, indeed, a later government investigation found serological evidence of infection in 33 of $56(58.9 \%)$ household members of infected poultry workers with no known exposure to infected birds (Du Ry van Beest Holle et al. 2005) with plausible second-generation human transmission (van Boven et al. 2007). This suggested an ease of intrafamilial avian influenza transmission on par with seasonal human influenza (Jennings and Miles 1978). Based on the seroprevalence of $\mathrm{H} 7$ antibodies, it was estimated that at least 1,000 and perhaps as many as 2,000 people were infected in the outbreak (Bosman et al. 2005).

In contrast with the four case reports of single human infections recorded prior to 1997, in the last decade hundreds of zoonotic avian influenza cases have been reported across four continents. In the Netherlands outbreak, apart from the one fatal case, most of the confirmed human H7N7 infections were relatively benign conjunctivitis or a mild flu-like illness (Fouchier et al. 2004), but human-to-human virus transmission seemed extraordinary. $\mathrm{H} 5 \mathrm{~N} 1$ has the inverse relationship-high case fatality but extremely limited human transmissibility. The 2003 Netherlands outbreak suggests avian influenza viruses may now directly, without recombination or adaptation, result in efficient human-to-human transmission; the human H5N1 cases starting in 1997 suggest that avian influenza viruses can directly be virulent zoonotic pathogens. Should the escalation of avian influenza outbreaks and human infections continue, both characteristics-extreme virulence and human transmissibility-may combine to produce a human pandemic of grave proportions. Describing the acceleration in size, scope, and frequency of highly pathogenic avian influenza virus outbreaks in recent years, llaria Capua told Science: "We've gone from a few snowflakes to an avalanche" (Enserink 2005). To slow this apparent rapid, recent emergence of zoonotic poultry influenza viruses, it must first be understood what triggered this "avalanche" in the first place.

Integrated rice-duck farming (Shortridge 1992), pig-hen-fish aquaculture (Scholtissek and Naylor 1988), and live poultry markets (Woo et al. 2006) have all been implicated in the emergence of avian influenza 
viruses with pandemic potential. The subsequent dissemination of viruses like H5N1 has been blamed on factors such as the songbird (Melville and Shortridge 2004), exotic bird (Borm et al. 2005) and cockfighting trades (Gilbert et al. 2006), migratory wild birds (Weber and Stilianakis 2007), and the trade in commercial poultry and poultry products (Peiris et al. 2007). None of these putative risk factors for emergence and spread are new, however. Domesticated ducks have been used as an adjunct to rice farming in Asia for centuries (Shortridge 2003a), fighting cocks (Scott 1983) and poultry have been traded for millennia, and wild birds have been migrating for millions of years (Proctor and Lynch 1993). The recent intensification of the global poultry sector may better account for the "complete revolution" (Alexander 2007) in the ecology and epidemiology of avian influenza over the last decade.

1c. Global Poultry Sector Intensification. Peiris et al. (2007) implicates the expansion of intensive poultry husbandry for facilitating the increasing scale and frequency of HPAI outbreaks around the world starting in the 1990s, a charge that seems to match temporally with the changes taking place in the global poultry industry. Although industrial production methods were invented in the United States in the 1950s (Boyd 2001), only in recent decades has intensive production truly globalized.

In the 1990s, the poultry industry in China experienced its fastest rate of growth and surpassed all others in poultry and egg industry expansion. By the time the Hong Kong H5N1 outbreak occurred in 1997, the production of domestic ducks, chickens, geese, and guinea fowl had quintupled over levels in 1980 (FAO 2007b), and production methods had changed dramatically. Indeed, in 1980, nearly all chickens were still raised in traditional backyard systems (FAO 1998); seventeen years later, when H5N1 emerged in Hong Kong, approximately half of the 10 billion poultry (FAO 2007b) produced in China were intensively confined (FAO 1998) in more than 60,000 intensive broiler chicken facilities, with a few operations raising more than 10 million chickens at a time (Simpson et al. 1999). Perhaps only a change this great-from small, outdoor, backyard flocks to intensive confinement in 10-million bird megafarms-could account for the dramatic shifts that occurred in the ecology and epidemiology of avian influenza during this same period.

Many of the analyses that have implicated poultry industry intensification in the changing evolution of avian influenza are presumably (Zanella 2002) or explicitly (Webster 2005) based on the assumption that, all things being equal, the greater the number of potential zoonotic influenza hosts, the greater the potential risk of zoonotic influenza transmission. Logically extended, this may further translate into greater risk of pandemic emergence, given, for example, that in China alone there are now an extra 10 billion potential vehicles every year for the genetic infusion of antigenically novel avian influenzavirus $A$ surface proteins into the human population.

This reasonable, if simplistic, notion could alone explain how the intensification of the global poultry sector could increase the risk of human pandemic emergence. Beyond this strictly stochastic model, though, there is evidence to suggest that Galliform species (i.e., terrestrial birds such as chickens, turkeys, and quail) may be particularly fit as intermediate hosts for the evolution of human pandemic strains in general, and perhaps virulent pandemic strains in particular.

\section{Bridging the Species Gap}

All influenzavirus A genes may have their origin in waterfowl and shorebirds, but there is apparently little zoonotic spread directly from this aquatic reservoir. Indeed, a mild case of conjunctivitis may be the most severe ailment caused by a wild duck virus in nature (Davis 2005). Human influenza viruses do not replicate efficiently in ducks (Hinshaw et al. 1983), and duck influenza viruses do not efficiently infect humans (Beare and Webster 1991). Compared to pig farmers, who may have 35 times the swine flu seroprevalence of those not occupationally exposed to pigs (Myers et al. 2006), virologic and serologic detection for avian influenza viruses among those handling wild ducks, such as duck hunters or wildlife professionals, have reportedly indicated few (Gill et al. 2006) if any (Beare andWebster 1991) cases of infection. Influenza viruses found in their natural reservoir do not seem to pose a significant zoonotic risk. As such, two billion waterfowl (FAO 2007b) being raised in China at the time of the 1997 Hong Kong outbreak may not have had direct impact on zoonotic risk, but there are two means by which avian 
influenza genes can migrate from their natural reservoir into the human species: reassortment or adaptation in an intermediate host.

One host range limiting factor that may help restrict avian influenza viruses towaterfowl is receptor specificity, which is a determinant of host cell virus attachment and virion release (Naeve et al. 1984). Influenza viruses bind to sialic ( $\mathrm{N}$-acetylneuramic) acid joined to a galactose of a host cell membraneanchored glycoprotein oligosaccharide in an $\alpha-2,3$ or $\alpha-2,6$ attachment. The intestinal epithelium of ducks presents $\alpha-2,3$ linkages ("bird-like"), to which waterfowl influenzavirus A has a high affinity, whereas viruses circulating in humans show a preference for $\alpha-2,6$ bonded terminal sialyl-galactosyl moieties ("human-like"), which are found in the human respiratory tract (Rogers and Paulson 1983).

The difference in receptor compatibility between duck enteric epithelium and human respiratory epithelium may require avian influenza viruses to replicate in an intermediate host to bridge this species gap (Webby andWebster 2001) via direct adaptation of a purely avian virus, as is believed to have occurred in the 1918 pandemic (Taubenberger et al. 2005), or a gene segment reassortment between avian and human strains responsible for the antigenic shifts in 1957 and 1968 (Scholtissek et al. 1978). Historically considered the most likely candidate for the role of intermediate host between ducks and humans has been the domestic pig (Scholtissek 1990).

2a. Pigs as Intermediate Hosts. Pigs have been postulated as a potential "mixing vessel" for gene segment reassortment due to the existence of both "bird-like" (®-2,3 linked) receptors and "human-like" ( $(2,6$ linked) receptors in porcine respiratory epithelium, a hypothesis supported by documentation of genetic reassortments between avian and human influenza viruses in both U.S. (Zhou et al. 1999) and European pigs (Castrucci et al. 1993). Human and avian viruses could then hypothetically co-infect and reassort within the same dual-receptor carrying porcine cell to create a hybrid virus with pandemic potential, i.e., recognizing "human-like" receptors but retaining enough avian antigenic novelty to escape pre-existing human immunity.

Evidence of serologic exposure to $\mathrm{H} 5 \mathrm{~N} 1$ in pigs in Vietnam (Choi et al. 2005a), combined with the enzootic presence of human H3N2 viruses (Sydney97-like) in pigs in southern China (Peiris et al. 2001), has raised concern that $\mathrm{H} 5 \mathrm{~N} 1$ could acquire human transmissibility in this way, but preliminary reassortments created artificially between $\mathrm{H} 5 \mathrm{~N} 1$ and $\mathrm{H} 3 \mathrm{~N} 2$ viruses failed to result in sustained transmission in a ferret model (Maines et al. 2006). Evidence that avian-like swine viruses may show a shift towards human-type receptor specificity over time suggests another, though not mutually exclusive, mechanism through which pigs could theoretically bridge the gap between the aquatic reservoir and human hosts, through adaptation rather than reassortment (Ito et al. 1998).

China's global dominance in animal agriculture is unparalleled and may indeed impact pandemic risk. The world's principal pork producer, reportedly possessing some of the largest industrial pig units in the world, confining as many as 250,000 pigs in single, six-story, concrete buildings (ZhichuuYan et al. 2003), China also produces more than $70 \%$ of the world's tonnage of duck meat and more than $90 \%$ of global goose meat (FAO 2007b). Given this annual amalgamation of hundreds of millions of pigs and billions of domestic waterfowl, China has been described as a nationwide "reassortment laboratory" for avian influenza viruses (Das 2002).

2b. Humans as Intermediate Hosts. The 1997 Hong Kong H5N1 outbreak demonstrating direct zoonotic transmission from chickens has been used to suggest a second model of pandemic emergence: humans themselves as intermediate hosts (Claas and Osterhaus 1998). There is indeed evidence now that the respiratory tracts of humans, like pigs, also harbor "bird-like" receptors, which questions the swinedependent transfection hypothesis (Matrosovich et al. 2004) and has been used to speculate on the origins of the 1918 pandemic (Taubenberger and Morens 2006). The lack of sustained human-to-human H5N1 transmission despite this dual receptor status continues to be attributed to the purported paucity of these "bird-like" $\alpha-2,3$ linked receptors in the upper human respiratory tract (Labadie et al. 2007), but this was based on increased $\alpha-2,3$ linkage-specific MAA2 lectin probe binding in lower versus upper human respiratory epithelium (Shinya et al. 2006). Subsequently, it was shown that H5N1 viruses could readily 
infect ex vivo human nasopharyngeal, adenoidal, and tonsillar epithelial tissue, questioning the reliance on MAA2 lectin histochemistry (Nicholls et al. 2007).

The scant current human transmissibility of $\mathrm{H} 5 \mathrm{~N} 1$, then, may be explained at other steps of viral replication (Subbarao and Katz 2000) and/or require more robust ${ }^{\circledR}-2,6$ linked receptor binding (Tumpey et al. 2007a). Based on studies of the resurrected 1918 pandemic virus, Tumpey et al. (2007a) showed that only modest changes in an influenzavirus $A$ hemagglutinin receptor binding site can switch preference between human-type and bird-type receptors. This raises concern that relatively minor genetic modifications could enhance H5N1 transmission to humans.

Given the presence of both avian and human-type receptors in the human respiratory tract, humans could theoretically act as their own mixing vessels for avian influenza viruses. Yet, as all influenza viruses originate in aquatic birds and again, based on studies of both human (Beare andWebster 1991) and nonhuman (Murphy et al. 1982) primates, duck viruses do not have the ability to efficiently infect humans, how does human infection with avian influenza occur? A "stepping stone" species within which to reassort or adapt may be needed.

It is a mistake to think of the class Aves as monolithic. Influenza viruses behave differently in Anseriform species (e.g., ducks, geese, swans) and Galliform species (e.g., chickens, turkeys, quail). The HongKong victims were apparently infected with H5N1 by chickens, not ducks (Shortridge 1999). In fact, nearly all human infections with avian influenza viruses in general have been associated with chickens (ECDC 2007). Rather than proposing humans as an intermediate host, the Hong Kong human outbreak should then have suggested a third model of pandemic emergence, though not mutually exclusive: chickens as intermediate hosts.

\section{2c. Gallinaceous Poultry as Intermidiate Hosts}

2c(1). Gallinaceous Intermediate Hosts: Reassortment. Just as "bird-like" influenza receptors were recently found in human lung tissue, "human-like" receptors have been found in chicken lung tissue (Feldmann et al. 2000). Gambaryan et al. (2002) demonstrated that while human influenza strains do not bind to duck epithelium preparations, they do bind to chicken epithelium. This may explain why mammalian influenza viruses do not tend to replicate in ducks, but may display limited growth in gallinaceous poultry species (Perez et al. 2003). This suggests that Galliform birds such as chickens may play the "mixing vessel" role once thought exclusive to pigs and result in the dissemination of avian/mammalian influenza reassortment viruses (Gambaryan et al. 2002).

The presence of $\alpha-2,6$ terminated receptors in chickens may explain, for example, how recent H9N2 viruses can continue to replicate in chickens despite their human virus-like receptor specificity (Gambaryan et al. 2003). Further molecular characterization of $₫-2,6$ linked "human-like" receptors in chickens and quail supports this hypothesis that gallinaceous poultry may act as intermediate hosts in the generation of antigenically new influenza viruses with pandemic potential (Guo et al. 2007).

Adding billions of chickens to the duck-pig "reasortment laboratory" in China may then compound risk. Meat production in China doubled in the 1980s and then doubled again in the 1990s (FAO 2006). This demand-driven increase in the industrialization of chicken and pig production manufactures billions of new potential zoonotic hosts for influenza every year. With per-capita meat consumption in China expected to increase an additional 45\%by 2020 (Delgado et al. 1999) with the Chinese population rising continuously until 2030 (Meng 2002), animal agriculture inventories will likely expand as well.

The rise in chicken consumption may be more concerning from a zoonotic influenza standpoint, if only for the fact that their bodies are so comparatively small. The recoverable meat per animal per year is so much greater in pigs than chickens, that equivalent increases in poultry consumption may result in the breeding of approximately 45 times more potentially susceptible hosts (FAO 1999). Although pork is the world's most popular meat (USDAFSIS 2003), the biggest piggeries still may only contain tens of thousands of animals (Simpson et al. 1999), whereas a single egg farm can confine more than one million animals (IEC 2002) and some broiler chicken facilities ten million (Simpson et al. 1999). It may take six 
months for a piglet to reach slaughter weight, but much of the global broiler chicken population is hatched and processed in as few as six weeks, dramatically multiplying the annual number of new potential zoonotic hosts. Given that more than 45 billion chickens pass through the world every year, along with 1 billion pigs, never before has the influenza virus had so many potential "stepping stones" from which to choose.

2c(2). Gallinaceous Poultry as Intermediate Hosts: Direct Adaptation. Evidence of certain influenzabinding similarities in the respiratory tracts of both chickens and primates, apart from dual terminal sialic acid linkage possession, speculatively suggests that influenza viruses may not only reassort in gallinaceous poultry, but may adapt to humans in chickens "by proxy." Comparing influenzavirus A binding to a variety of primate, chicken, and duck epithelial samples, Gambaryan et al. (2002) found that both human and chicken strains preferably bound to the gangliosides with long sugar chains found in monkey and chicken tissue, rather than the shorter sugar chain gangliosides abundant in duck intestine that the duck strains preferred. Although the role of ganglioside receptors remains unclear (Matrosovich et al. 2006), this suggests that the spectrum of influenza receptor sialylglycoconjugates in chickens is more similar to that found on primate respiratory epithelium than duck epithelium, emphasizing the potential role chickens could play as intermediate host in the adaptation and transmission of influenza viruses from ducks to humans.

Subsequent testing of binding affinities of a variety of duck, chicken, and human influenza viruses to a wide array of well-defined receptor analogs led Gambaryan et al. (2003) to speculate that receptor-level similarities found in chicken and monkey epithelium may facilitate the infection of primate cells with chicken viruses. It remains to be seen if further similarities found between chicken and human virus binding to complex variations of carbohydrate structure (Gambaryan et al. 2004) such as gangliosides and sulfated and fucosylated oligosaccharides (Gambaryan et al. 2005) facilitate the transmission or adaptation of duck viruses to mammals. At a minimum, oversimplistic models of host restriction may need reassessment (Stevens et al. 2006).

2c(3). Gallinaceous Poultry as Intermediate Hosts: Exaptive Adaptation. A single amino acid change can affect host-species specificity, as demonstrated with influenzavirus A H5N1 (Gambaryan et al. 2006) and the 1918 pandemic virus (Glaser et al. 2005), as well as SARS Co-V (Yi et al. 2005). Receptor specificity, however, is but one potential determinant of host restriction. Avian H9N2 viruses can infect humans, for example, but despite "human-like" receptor specificity, no human-to-human transmission has been detected (Uyeki et al. 2002). Fusing into a host cell is only the first stage of infection. Influenza viruses must then successfully co-opt cellular machinery for replication (Gabriel et al. 2005).

To rapidly acquire adaptive mutations, pathogens first need the necessary replicative machinery. RNA viruses such as influenza tend to have high mutation rates as they, unlike DNA viruses, cannot take advantage of the proofreading abilities of host cell DNA polymerases. Mutation rates are so high that each of the millions of new influenzavirus $A$ progeny budding from host cells may have a distinctive genetic complement (Page and Holmes 1998). RNA virus populations, then, tend not to exist as a distinct genotypic class, but as viral quasispecies, a dynamic swarm of heterogeneous mutants, even within a single host (Domingo et al. 1985). As variability may be considered the engine of evolution, viruses that exist as quasispecies may have a plasticity to quickly adapt as new selection pressures arise (Domingo et al. 1998). This biological flexibility to mutate rapidly, evolve, and potentially adapt to new hosts (Holland et al. 1991), makes them prime candidates for host-switching pathogens. Indeed, a disproportionate number of emerging and reemerging zoonoses are RNA viruses (Taylor et al. 2001). Beyond the inability to repair genetic "errors" during replication, the segmented nature of the influenzavirus A genome allows for rapid evolutionary "shifts" via reassortment (Webster et al. 1982). No other human respiratory virus is thought to match the mutation rate of the influenza virus (McNamara 2002), which may account for its ability to infect such a wide range of hosts in so many varied environments.

The mutation rate of influenzavirus $A$ is determined by the viral polymerase complex which replicates the viral genome, allowing the virus to replicate. Mutations in the polymerase genes themselves-so-called "mutator mutations"-may play a role in determining both virulence and host switching abilities by, potentially, ramping up mutation rates even higher. There is a certain mutant viral polymerase subunit 
with an associated replicative power and temperature-dependence that may, respectively, increase mammalian virulence and "pre-adapt" avian influenza viruses for human infection.

Comparing the sequence of the H7N7 virus isolated from the fatal human case in the Netherlands outbreak with an H7N7 isolate from a human conjunctivitis case, the major determinant of virulence in a mouse model was the lysine at position 627 of viral polymerase subunit PB2 (Munster et al. 2007). This trait is shared by the 1918 pandemic virus and all subsequent human influenza $A$ virus lineages, as well as recent mammalian H5N1 isolates, but lacking in nearly all avian virus PB2 sequences (Chen et al. 2006a), suggesting it plays a key role in efficient human virus replication. The recent isolation of an HPAI H7N1 virus possessing PB2-627K in a naturally infected ostrich, along with the acquisition of a PB2-627 $\mathrm{E}$ to $\mathrm{K}$ mutation in an HPAI H7N1 chicken strain after a single passage in mice, suggest a genetic predisposition in mammals towards virulence of HPAI viruses found in terrestrial birds other than H5N1 and H7N7 (Rigoni et al. 2007). This suggests that adaptation to poultry may not only increase the human transmissibility of avian influenza viruses, but virulence as well.

Changing from a harmless, enteric, waterborne infection to a virulent, respiratory, and potentially even airborne infection requires not only adjustment to the intracellular environment of the host, but the extracellular environment as well (Hollenbeck 2005). Duck influenza viruses, for example, show improved infective stability at a lower $\mathrm{pH}$ compared to human-adapted strains. Despite the potentially higher alkalinity of fresh chicken feces compared with duck feces (Himathongkham and Riemann 1999), there is no indication that any changes in $\mathrm{pH}$ stability that might result from Galliform adaptation would have any effect on human infectivity (Webster et al. 1978). This may not be the case, though, with changes in temperature accommodation mediated by the viral polymerase subunit PB2.

Optimal avian influenza virus replication occurs at different temperatures in humans and birds. Avian influenza viruses replicate better at $41^{\circ} \mathrm{C}$, the temperature of the avian intestinal tract, whereas human influenza viruses replicate better at $33^{\circ} \mathrm{C}$, the temperature of the human respiratory tract. This temperature-dependent restriction may account for the ability of human influenzavirus $A$ (Memphis/110/76) to replicate in the respiratory, but not intestinal, tracts of ducks and may therefore be one component of the avian/human species barrier (Webster et al. 1978). Massin et al. (2001) determined that PB2 seems to play a role in the temperature sensitivity of viral RNA polymerase replication. This leads to speculation that by adapting to the lower temperature of the respiratory tract of Galliform poultry, avian influenza viruses from the waterfowl reservoir may be simultaneously improving replication in the upper airways of humans, which, incidentally, share the approximate temperature (Jones et al. 2005) and relative humidity (Heyder 2004) of broiler chicken sheds wherein the virus may be evolving.

This may prove to be an example of exaptation, in which an adaptation in one context coincidentally predisposes evolutionary success in an unrelated context, as was the case with Legionnaire's disease (Wolfe et al. 2000). Legionnaire's is caused by bacteria whose primary evolutionary niche is the scum lining the rocks of natural hot springs, a warm, moist environment not unlike the human lung. The invention of machines that could mist the air with water afforded an opportunity, as evidenced by the 1976 American Legion convention outbreak in Philadelphia, for ventilation systems to condition the air with bacteria now known as Legionella (Garrett 1994). In the environment of the human respiratory tract, Legionella's prior adaptations proved lethal in 29 of 182 cases (Fraser et al. 1977).

In the case of influenza, gallinaceous poultry may then, speculatively, be thought to act as both hot springs and air conditioner, providing both the media by which the virus can adapt and propagate to the human population. The evolutionary distance from duck to human may be too great for influenzavirus $A$ to switch hosts directly, but with chickens purportedly sharing cellular and extracellular milieu characteristics of each, they may act as "stepping stones" to bridge the species gap (Campitelli et al. 2004).

2c(4). Gallinaceous Intermediate Hosts: Rerouting of Transmission. Serological evidence of exposure to all avian hemagglutinin subtypes tested $(\mathrm{H} 4$ to $\mathrm{H} 13)$ in rural inhabitants of southern China suggests contact with avian influenza viruses is not uncommon in the region (Shortridge 1992). The presumptive progression of H5N1 from aquatic birds (ducks) to partially aquatic birds (geese) to terrestial birds (chickens) to terrestial mammals (humans) (Shortridge et al. 2003) led Shortridge (2003a) to speculate 
that the global intensification of the poultry industry was a key element in adapting avian influenza viruses to terrestrial species.

In a terrestrial host, avian influenza viruses cannot be effortlessly spread via fecal-water-oral transmission (i.e., chickens don't swim). Acclimatization to land-based birds may result in selective adaptations in avian influenza viruses that may make them more adaptive to terrestrial mammals as well. Any modification that enhances airborne transmission, for example, such as a shift from intestinal to respiratory replication, could potentially amplify zoonotic risk. Influenza viruses infecting chickens from the intestines of aquatic birds may indeed be under selective pressure to switch from replicating in the gut to replicating in the lungs, but is there evidence of this actually happening?

The initial H5N1 outbreak in Hong Kong in 1997 was extinguished by culling more than a million birds (Shortridge et al. 2000). When H5N1 viruses re-emerged in Hong Kong in 2001, they were pathogenic not only to chickens (Guan et al. 2002) and humans (Wuethrich 2003a), but, for the first time ever recorded, pathogenic to a range of wild waterfowl as well. More than 100 ducks, geese, swans, flamingoes, and overwintering feral migratory birds died at local wildlife parks (Sturm-Ramirez et al. 2004). This was unprecedented. Influenzavirus A infection in aquatic birds had, with few exceptions (Becker 1966; Capua and Mutinelli 2001) been known to be asymptomatic (Webster et al. 1992).

As expected, the H5N1 virus isolated from the first human case in 1997, presumably soon after the virus jumped from waterfowl to chickens (Chen et al. 2004), was essentially harmless in ducks (Perkins and Swayne 2002). Sturm-Ramirez et al. (2005), however, demonstrated experimentally that some H5N1 viruses isolated from chickens after 2002 were highly lethal in ducks, showing that H5N1 may have disrupted the longstanding evolutionary "equilibrium" between influenza viruses and aquatic birds. Not only may the ecology of avian influenza be changing, but the biology as well (Hulse-Post et al. 2005). Comparisons with $1997 \mathrm{H} 5 \mathrm{~N} 1$ isolates showed diverse reactivity patterns that suggest considerable antigenic drift over the preceding years. One of the most significant changes noted, though, was a shift from predominantly cloacal shedding to predominantly tracheal shedding, suggesting that the main path of transmission of H5N1 was shifting from an oral-fecal route to more of an oral-oral or even airborne route. Furthermore, the degree of pathogenicity in ducks was associated with the virus titers recovered from the birds' tracheas, suggesting this shift may be a factor in the increased pathogenicity in certain post-2002 isolates.

H5N1 therefore seemed to enter chicken populations as an intestinal virus, but left as more of a respiratory virus. This phenomenon has also been demonstrated experimentally. When a wide range of wild aquatic bird viruses were experimentally transferred to quail, the preferred site of replication switched from the intestinal epithelium to predominantly the respiratory tract (Mararova et al. 2003). This putative move away from enteric transmission suggests gallinaceous birds may be the "route modulator" responsible for changing the fecal-oral route of infection to respiratory aerosol transmission.

Terrestrial birds are not natural hosts for influenza (Suarez et al. 1999), but the receptors they bear may be recognizable enough by duck influenza viruses to support infection. Likewise, though chickens may still be heterologous hosts for humans, adaptation to replication in a homologous organ system-the respiratory tract, rather than Anseriform enteric columnar epithelium (Hinshaw et al. 1980a)-suggests that adaptation of duck viruses to chickens may result in a concomitant enhanced transmissibility to humans (Murphy et al. 1982). The critical crossover from waterborne intestinal virus to potentially airborne human respiratory pathogen may take place in chickens and other terrestrial poultry.

A pandemic virus faces a paradoxical twin challenge: It must be relatively new to the human immune system so there is little pre-existing immunity while, at the same time, being supremely well-adapted to infect and replicate within human tissue (Reid et al. 2001b). The hypothesis that gallinaceous poultry may enhance the zoonotic and, hence, possibly the pandemic risk of avian influenza via some level of "preadaptation" to human infectivity is supported by recent H5N1 and H9N2 isolates with increased human receptor binding capacity (Gambaryan et al. 2006), a seeming shift from H5N1 enteric to respiratory shedding, and a growing body of evidence demonstrating influenzavirus A receptor binding similarities in chickens and humans. As aquatic avian influenza viruses become better adapted at 
infecting chickens, they may to some degree become better adapted at infecting humans. Webster (2005) and others may then be correct in implicating the global intensification of poultry production for the ecologic and epidemiologic shift in avian influenza in the 1990s and whatever subsequent future pandemic risk this may bring.

3a. HPAl and Pandemic Emergence. When certain waterfowl influenza virus subtypes- $\mathrm{H} 5$ and $\mathrm{H} 7$ viruses-infect gallinaceous poultry, these viruses may, in certain circumstances, unpredictably mutate into highly pathogenic strains that by definition have the potential to replicate throughout the bodies of infected birds, resulting in potentially devastating epizootics. The H5N1 strain that arose in Hong Kong in 1997 and has since spread around much of the world, resulting in the deaths of 200 people and 200 million birds, is one such example of an HPAI virus. This capacity of HPAI viruses to cause systemic infection beyond the respiratory or intestinal tract in gallinaceous poultry may result in increased avian-toavian or avian-to-human infection which may in turn lead to an elevated risk of pandemic emergence.

Known sporadically for more than a century as "fowl plague," highly pathogenic avian influenza (HPAl) strains have been defined as producing a minimum of $75 \%$ mortality in chickens under defined experimental conditions (Bankowski 1981). All HPAl viruses seem to start as low pathogenicity avian influenza (LPAI) viruses. The current understanding is that HPAI viruses typically arise by mutation from LPAI viruses introduced into terrestial poultry (Alexander 2007). Phylogenetic studies show that HPAl viruses do not constitute separate lineages, but instead arise from nonpathogenic strains (Rohm et al. 1995) by increasingly better understood mechanisms (Horimoto and Kawaoka 2001).

For all influenza viruses to become infectious, the membrane fusion-mediating glycoprotein, hemagglutinin, requires a proteolytic activation step. The precursor hemagglutinin (HA) protein must first be cleaved in two to allow for viral entry into host cells (Rott 1992). Since the influenza virus genome does not contain a protease for HA, the virus is dependent on host enzymes, raising, incidentally, the possibility of the future development of anti-influenza combination therapy incorporating host protease inhibitors (Kido et al. 2007). LPAl viruses typically possess only a single basic amino acid, arginine, at the cleavage site and, as such, require exogenous "trypsin-like" enzymes for cleavage, effectively restricting virus replication to tissues containing this enzyme, i.e., respiratory and intestinal epithelium (Bosch et al. 1979). The HA precursor cleavage sites of HPAI viruses, on the other hand, contain multiple upstream basic amino acids allowing for cleavage by ubiquitously distributed subtilisin-like endoproteases such as furin (Stieneke-Grober et al. 1992) and PC6 (Horimoto et al. 1994), enabling the virus to invade the submucosa, vasculature, and lymphatics to ultimately replicate systemically in the visceral organs, brain, and skin of infected birds. This causes a variety of congestive hemorrhagic, transudative, and necrobiotic changes, resulting in up to nearly $100 \%$ mortality within a week (Hofstaad et al. 1972). Hemagglutinin cleavability, then, is one of the major determinants of both tissue tropism and virulence in poultry.

HPAI virus outbreaks erupt when a low-pathogenicity precurser $\mathrm{H} 5$ and $\mathrm{H} 7$ subtype virus is introduced into a domestic poultry flock and acquires mutations to confer high poultry pathogenicity. This is supported by an in vitro demonstration of mutation to virulence ( $\mathrm{Li}$ et al. 1990), as well as serial passaging studies in which an avirulent H5N3 wild swan field isolate repeatedly passaged through chickens incrementally acquired HA cleavage site basic amino acids and expanded tissue tropism to culminate in pantropicity and a $100 \%$ mortality rate (Ito et al. 2001).

Approximately $98 \%$ of all reported cases of avian influenza virus infection in humans and $100 \%$ of the reported deaths have been caused by HPAI strains (Bosman et al. 2005; ECDC 2007). This has led to speculation that HPAl viruses may intrinsically pose a greater zoonotic threat (CDC2006). The actual proportion of HPAI to LPAI human infections is likely much lower because of the tendency of LPAI strains to cause asymptomatic infection and thereby go unrecorded, but this may just further support the notion.

Certain characteristics of HPAl viruses may increase the risk of avian spread. Compared to LPAI infections, HPAI may result in greater (Pantin-Jackwood et al. 2007) and more prolonged (Hulse-Post et al. 2005) shedding of virus from a multiplicity of routes (Sturm-Ramirez et al. 2005). A combination of these factors may cause explosive spread within industrial poultry units (FAO 2007a), by turning infected poultry into virtual "virus pumps" (Dieraur) contaminating the local environment, facilitating infection within 
and between poultry populations, and amplifying the virus to levels that overwhelm tracing and culling capacities (Marangon et al. 2003), making biosecurity virtually impossible (Capua and Marangon 2003a).

HPAI virus may be present in the eggs (Swayne and Beck 2004), blood, bones, skin, and meat of infected poultry (Swayne and Beck 2005). The H5N1 strain has reportedly been demonstrated in the meat of infected chickens, quail, ducks, and geese (Swayne 2006), and virus may be found in both dark and white meat (Swayne and Beck 2005). The expansive range of susceptible host tissue could potentially increase avian spread via the international trade in poultry and poultry products if discarded uncooked meat scraps, for example, are fed to poultry (Sims et al. 2005). Infectious and highly pathogenic H5N1 isolates have been repeatedly (Tumpey et al. 2002) recovered from frozen duck meat exported from China processed for human consumption (Mase et al. 2005), and Swayne and Beck (2005) showed that feeding chickens meat from H5N1-infected chickens resulted in fatal infection, suggesting that the purported enhanced trade-related risk of HPAI viruses may be a genuine concern. Although surface contamination of meat from respiratory or intestinal tissue may occur during poultry processing, LPAI viruses have failed to be identified in the skeletal muscle of chickens (Mo et al. 1997a) and therefore probably do not carry this risk (Zepeda and Salman 2007). The discovery of H5N1 muscle infection in clinically normal birds underscores this possible mode by which HPAl viruses could potentially increase the risk of spread to distant poultry populations (Tumpey et al. 2002).

This same trait, pantrophic tissue distribution, may also increase the risk of avian influenza infection in humans and other mammals. The presence of HPAl viruses in the bodily fluids of birds raised for slaughter (Swayne and Beck 2005), for example, may make handing and butchering sick poultry a source of human infection (Beigel et al. 2005). Cats and other carnivores can suffer fatalities after eating infected chickens (Thiry et al. 2007), and pigs fed eggs from an infected flock have fallen ill (IFSAN 2004). The available evidence also suggests that the gastrointestinal tract in humans could also be a portal of entry for H5N1 virus (Butler 2006a), though proper handling and cooking would eliminate any potential food safety risk (Greiner et al. 2007). No virus was recovered from meat samples from infected chickens after cooking at $70 \pm \mathrm{C}$ for just five seconds (Thomas and Swayne 2007), though commercial methods used to pasteurize dried egg products may not eliminate HPAI viruses (Swayne and Beck 2004).

The preponderance of human cases and fatalities attributed to HPAI viruses, coupled with the potential for enhanced avian, mammalian, and human spread of infection, arguably support a case thatHPAI viruses might increase the risk of pandemic emergence. The appearance of the veritable panzoonotic of LPAI H9N2 in Eurasian chickens, though, suggests that Galliform poultry infections in general may have an increased capacity to spread. Although H5N1 virus has spread rapidly throughout much of the world, the same severity of HPAI poultry outbreaks that may make them difficult to control also facilitates detection. Epizootic LPAI outbreaks in poultry, in contrast, may be overlooked and, as such, have great potential for avian spread, given the limited veterinary surveillance infrastructure in many countries. The same could be said for H9N2 human infection.

The five human cases discovered in Guangdong province (Guo et al. 1999) and the three children found to be infected in Hong Kong SAR in 1999 (Peiris et al. 1999) and 2003 (Butt et al. 2005) evidently suffered relatively mild, self-limited illnesses clinically indistinguishable from seasonal influenza (Butt et al. 2005). Given the human-like receptor specificity of these viruses (Gambaryan et al. 2003), this raises at least the theoretical possibility that a human-adapted LPAI virus could escape detection and potentially circulate further into the human population than an HPAI virus, which may be associated with more severe, and thereby more noticeable and perhaps more controllable, human infection.

Although LPAI outbreaks may be more likely to escape detection in general, certain H5N1 viruses have the demonstrated capacity to cause asymptomatic infections in ducks with prolonged shedding of virus that retains high pathogenicity to chickens and presumably to humans as well-the source of Hulse-Post et al.'s (2000) "Trojan duck" hypothesis. The HPAI H5N1 virus might then combine devastating outbreaks and silent spread.

Similarly, H9N2 infection has been detected in pigs (Peiris et al. 2001), but does not seem to have the mammalian scope attributed to H5N1. Though human H9N2 cases may be missed, there is no evidence 
of human-to-human H9N2 infection (Uyeki et al. 2002). H5N1 may likewise almost entirely lack human-tohuman transmissibility (Beigel et al. 2005), yet the most efficient avian influenza human-to-human spread to datewas documented in the Netherlands outbreak of H7N7, an HPAI virus.

Thus, HPAI viruses may be associated with a higher pandemic risk, but not necessarily so. Since but for a few exceptions (Becker 1966; Sabirovic 2004), HPAI viruses only occur in gallinaceous poultry, one might more parsimoniously merge the apparent increased epizootic and zoonotic risk linked to both LPAI H9N2 and HPAI viruses including H5N1, by speculating an increased risk of pandemic emergence associated with epizootics involving Galliform species in general. Recent evidence of two-way interspecies transmission of $\mathrm{H} 5 \mathrm{~N} 1$ and $\mathrm{H} 9 \mathrm{~N} 2$ viruses between terrestrial poultry underscores the protean pandemic threat (Xu et al. 2007).

3b. HPAI and Pandemic Virulence. Given the human pathogenicity associated with H5N1 and H7N7 infection, might there also be the potential for increased virulence of a pandemic strain that arose from either zoonotic Galliform strains in general or HPAl strains in particular? It might seem selfevident that a pandemic influenza virus with the potential for trypsin-independent activation-as is thought inherent to HPAI viruses-would pose a greater threat, as this would presumably allow the virus to escape enzyme restriction to the respiratory tract to replicate in the brain and other vital organs (Kristensson 2006). We know that HPAl viruses may be highly pathogenic for poultry, but evidence supporting the presumption that HPAI viruses may be more pathogenic to people as well is evocative, but not yet conclusive.

Similar to the clinical course of the fatal human case of HPAI H7N7, human HPAI H5N1 infections are characterized by fulminant pneumonia as well as multiorgan failure and often death (Arabi et al. 2007). Extrapulmonary clinical manifestations and infectious virus or viral RNA recovery have been documented in a number of cases of H5N1 infection in humans (Uiprasertkul et al. 2005). The clinical and virologic evidence in one H5N1 case series in particular-a four-year-old boy and his nine-year-old sister, both presenting with no apparent respiratory disease, only severe diarrhea, followed by rapidly progressive coma and death-is very suggestive of systemic infection. H5N1 virus was isolated not only from the throat, but also the rectum, serum, and cerebrospinal fluid (de Jong et al. 2005).

There have been sporadic encephalopathy cases (Morishima et al. 2002), cerebrospinal fluid influenzavirus A isolation (Fujimoto et al. 1998), and viremia (Lehmann and Gust 1971) reported in severe cases of seasonal influenza, but they are considered rare. Limited postmortem immunohistochemical analyses in human $\mathrm{H} 5 \mathrm{~N} 1$ cases only found evidence of viral replication in lung and intestine (Uiprasertkul et al. 2005), but the relative lack of virus antigen-positive cells may be a consequence of death in the late stages of disease after intensive antiviral treatment (To et al. 2001).

The animal model data are mixed. Necropsies of certain other H5N1-affected mammals-mice (Nishimura et al. 2000), ferrets (Govorkova et al. 2005), felids (Keawcharoen et al. 2004), and viverrids (Roberton et al. 2006) - are clear: widely disseminated multi-organ involvement indicative of permissive mammalian tissue tropism. In a mouse model in particular, highH5N1 hemagglutinin cleavability was found an essential requirement for lethal infection (Hatta et al. 2001).

Comparing the effect of inoculation routes using human H5N1 virus isolates, Bright et al. (2003) demonstrated in an inbred mouse model (BALB/C mice) that extrapulmonary H5N1 virus spread and replication were consistently correlated with lethality. When an H5N1 virus isolated from a fatal human case (HK/483) was intranasally inoculated in mice, the virus spread systemically, replicated in multiple organs including the brain, and proved $100 \%$ lethal. When a different $\mathrm{H} 5 \mathrm{~N} 1$ virus, HK/486, isolated from a child exhibiting mild illness (Lu et al. 1999) was intranasally infected in mice, virus replication remained restricted to the respiratory tract andwas generally nonlethal. If the low pathogenicity phenotype virus is intracerebrally inoculated in the mice, however, the result is $100 \%$ mortality. This suggests that not only is systemic spread occurring, but that wider tissue tropism is determinant of virulence in mammals (Bright et al. 2003). In a ferret model, though, intranasal infection of either H5N1 virus could result in systemic spread, disease, and death (Rowe et al. 2003). 
Although some consider mice a good model for human H5N1 infection and lethality (Webster 2001), others suggest that the lack of the interferon-inducible gene $\mathrm{Mx} 1$ in standard laboratory mice-which might otherwise provide protection-may present a caveat to murine extrapolation (Tumpey et al. 2007b). Notably, pathology seems restricted to the respiratory tract in experimentally infected cynomolgus macaques (Rimmelzwaan et al. 2001).

Simply because an HPAl virus is by definition highly pathogenic, or potentially highly pathogenic to chickens, does not necessarily mean it is pathogenic to other hosts. There is even variation among bird species (Sturm-Ramirez et al. 2005). H5N1 virus, though, is uncontestedly highly pathogenic to humans. Never before has a human influenzavirusAinfection been documented to share the combination of viral load kinetics, dissemination, case fatality, and hypercytokinemia induction found in H5N1 infection (de Jong et al. 2006). At least part of this virulence, based on the suggestive clinical, virologic, and animal model data, may be due to HPAl virus trypsin-independent activation (Alexander et al. 2003).

The initial human H5N1 outbreak in 1997 was described as the first time an avian influenza had resulted in human respiratory infection and death (Claas et al.1998), but that was before Taubenberger et al. (2005) published the complete sequence and final phylogenetic analysis of the genome of the 1918-1919 pandemic influenza virus that showed it likely to have been a mammalian-adapted avian influenza virus as well. The 1997 Hong Kong outbreak did suggest a possible explanation for the virulence of the 1918 pandemic: Perhaps the 1918 virus had a ubiquitously cleavable hemagglutinin like modern-day HPAl viruses.

A contemporary autopsy series in 1918 found sufficiently common and widespread extrapulmonary pathological changes to support the view of systemic disease during the pandemic, but inconsistent findings, relative restriction to hematopoetic organs and the vasculature, and no histopathological evidence of the widespread necrotic lesions seen in poultry HPAl infections were evident (Winternitz et al. 1920). This suggests that the extrapulmonary manifestations may have more been a result of collateral immunological damage than a direct effect of the virus.

A global epidemic of post-encephalitic Parkinsonism in the 1920s, encephalitis lethargica, was blamed on the 1918 influenza pandemic (Ravenholt and Foege 1982). This would be consistent with direct viral damage to the brains of pandemic survivors, but attempts to isolate influenza RNA from archived brains of encephalitis lethargica patients have failed (McCall et al. 2001) and no other such proof of a relationship has been obtained (Reid et al. 2001a).

The lack of a multibasic hemagglutinin cleavage site evident in the 1918 pandemic virus sequence seemed to put to rest the hypothesis that the virulence of the avian-like 1918 pandemic virus was akin to that of HPAI H5N1 (Taubenberger et al. 1997) until the 1918 pandemic virus was reconstructed using reverse genetics and found to have trypsin-independent activation after all, perhaps due to some sort of protease sequestion mechanism similar to that described by Goto and Kawaoka (1998). Though not yet tested on poultry, pathogenicity studies of the resurrected 1918 virus hemagglutinin gene suggest it would likely be classified as an HPAl virus despite the lack of multibasic cleavage site, given its trypsinindependent activation and high virulence both in a mouse model and an embryonated egg model of pathogenicity (Tumpey et al. 2005).

Even though there was no significant necrobiotic extrapulmonary pathology, the massive pulmonary hemorrhagic edema seen in a subset of 1918 victims does suggest a direct cytotoxic effect. Taubenberger (1998) speculated that the trypsin-independence of the pandemic virus may have contributed to virulence in the respiratory tract by, for example, having the capacity to more excessively damage respiratory vascular endothelium. Small changes in HA cleavability may have large effects on the virulence of human influenza, as seen in cases of bacterial pneumonia co-infection, which is thought to increase the pathogenicity of influenza via the bacterial secretion of trypsin-like proteases, thereby improving influenza virus replication (Tashiro et al. 1987).

The two most virulent knowninfluenza viruses, H5N1 and the 1918 pandemic virus, both share trypsinindependent hemagglutinin activation. In comparison, the relatively mild 1957 and 1968 pandemic 
viruses, thought caused by reassortments with LPAI viruses (Butt et al. 2005), did not. This supports the speculation that the hemagglutinin cleavability associated with HPAl viruses may give them the potential to trigger particularly virulent pandemics. If the 1918 influenza pandemic was caused by a virus restricted to the respiratory tract, then the morbidity and mortality associated with a pandemic caused by a virus capable of multi-organ system replication are unimaginable. H5N1 raises just such a specter of a potentially pantropic pandemic virus.

Based on a U.S. Public Health Service survey, the 1918 pandemic case fatality was estimated at $2.5 \%$ (Frost 1920). Currently, the case fatality of confirmed human H5N1 cases apparently exceeds $50 \%$ (WHO 2007), and a seroprevalence study among those with regular intense contact with sick poultry found no evidence of subclinical infection, suggesting this estimate may not be far exaggerated by biased case detection (Vong et al. 2006).Webster et al. (2006) described the pandemic potential of H5N1 virus as comparable to that of a "global tsunami."

It is impossible to predetermine if the H5N1 virus has the biological capacity to lead to a human pandemic. The focus of concern surrounding a potential H5N1 pandemic is not on its inevitability but its potential severity. Even if the actual case fatality is $>50 \%$ and the $\mathrm{H} 5 \mathrm{~N} 1$ virus was able to cause a pandemic, might not its pathogenicity fall as transmissibility rises? A working group convened by the WHO to answer such questions concluded that if H5N1 were to reassort with a human virus, akin to 1957 and 1968 pandemic emergence, then one might indeed expect the case fatality of the virus to decline. If, however, H5N1 should follow the putative path of the 1918 pandemic (Taubenberger et al. 2006) and acquire sustained human-to-human transmission through adaptation rather than reassortment, then the present lethality could be maintained during a pandemic (WHO 2006b). However, H5N1 has not yet caused a pandemic in its decade of existence, raising the question as to whether the HPAl can be withdrawn $\mathrm{H} 5 \mathrm{~N} 1$ from the pool of potential pandemic candidates.

Different genotypes of H5N1 have repeatedly infected humans for ten years without acquiring sustained human transmissibility, but this may not provide solace, given our incomplete understanding of influenza virus adaptation (Perdue and Swayne 2005), as well as events such as the sudden appearance of H3N8 canine influenza in the United States in 2004, the result of an unprecedented interspecies transmission of a wholly equine influenza virus (Crawford et al. 2005) that had previously been circulating uneventfully in U.S. horse populations for more than 40 years (Waddell et al. 1963). Rare constellations of genetic events may yet occur in the H5N1 virus given the vast replicative and mutable capacity of influenza viruses in general (Domingo et al. 1985) and the complex contemporary ecology and epidemiology of H5N1 in particular (Webster et al. 2007). Widespread enzoonicity in poultry populations of H5N1 (Smith et al. 2006) and H9N2 (Cameron et al. 2000) viruses may make timely eradication impractical.

3c. LPAI to HPAI. In the last decade, there has been an estimated hundred-fold increase in the number of birds (Capua and Alexander 2004) and humans (WHO 2007) affected by HPAl viruses. Pandemic concerns aside, HPAl outbreaks have potential social (Cristalli and Capua 2007), economic (Clague et al. 2006), occupational (Swayne 2006), agricultural (Spala et al. 2006), trade (Domenech et al. 2006), public health (Beigel et al. 2005), veterinary (Capua and Alexander 2006b), animal conservation (Roberton et al. 2006), and welfare (Serratosa et al. 2007) implications.

When an LPAI H5 or $\mathrm{H} 7$ virus from the aquatic bird reservoir is introduced into a population of terrestrial poultry either through a live bird market interaction (Senne et al. 2003) or similar biosecurity breach (Capua and Marangon 2000), the LPAI virus may, within weeks (Rojas et al. 2002) or months (Naeem et al. 2007), unpredictably mutate into anHPAl virus (Capua and Marangon 2003b). By their nature, LPAI viruses may be difficult to detect, but, in a few cases, the avirulent progenitors of HPAI outbreaks have been identified before the transformation, as with outbreaks in Pennsylvania starting in 1983 (Kawaoka and Webster 1985), Mexico in 1994 (Horimoto et al. 1995), Italy in 1999 (Capua et al. 2000), Chile in 2002 (Suarez et al. 2004), Pakistan in 2003 (Naeem et al. 2007), and Canada in 2004 (Power 2005), offering opportunities to study both the genetic changes that take place and risk factors for emergence.

Molecularly, the mutations accounting for this shift in pathogenicity have involved the formation or unmasking of the multibasic HA cleavage via a change in glycosylation pattern (Kawaoka et al. 1984), a 
nucleotide substitution (Perdue et al. 1997), or a nucleotide insertion, either via the spontaneous duplication of purine triplets (Garcia et al. 1996) or intersegmental RNA recombination-as was apparent in the Chilean (Suarez et al. 2004) and Canadian (Pasick et al. 2005) outbreaks. But what are the evolutionary pressures that select for HPAl transformation?

In the 1994 HPAl outbreak in Mexico, Horimoto et al. (1995) performed a phylogenetic analysis of isolates displaying varying degrees of pathogenicity and demonstrated for the first time in nature a seeming stepwise acquisition of virulence. The best studied example, though, was in Italy in 1999-2000 where an LPAI virus entering an industrial poultry production area mutated into an HPAI virus that killed more than 13 million birds (Capua et al. 2000). Banks et al. (2001) constructed a timeline of genetic changes showing that a single presumed waterfowl introduction may have seeded nearly 200 LPAl outbreaks before the virus evolved to virulence in a multiplicity of diverse locations contemporaneously, which suggests similar selective pressures existed to adapt the virus to its new host, terrestrial poultry.

In all cases in which the LPAI progenitor has been identified, the mutation to virulence has occurred in an intensive confinement setting. Some of the farms in which this transformation took place were massive600,000 broiler breeders on the farm in the Chilean outbreak (Rojas et al. 2002), for example, and 700,000 caged laying hens at the index farm in Pakistan (Naeem et al. 2007). This association between the emergence of HPAl viruses and intensive, as opposed to extensive (pasture-based), production is consistent with existing biological theory on the evolution of virulence.

\section{Poultry Intensification and Selection for Virulence}

Given that the emergence of HPAI viruses has almost always been associated with large-scale poultry production, a hypothesis can be generated that the conditions associated with intensive poultry systems may facilitate the emergence of HPAI viruses.

The WHO/FAO/OIE (2004) joint consultation on emerging zoonotic diseases noted that the sheer number of intense contacts between birds in intensive production serves to spread and amplify disease agents such as avian influenzavirus A. Even if the probability of transformation from a low pathogenicity strain is intrinsically low in a single cycle of replication, as the number of cycles increase as the virus spreads through a large population of densely confined susceptible hosts, the probability of rare genetic events may necessarily increase (Cauthen 2007).

Field studies of red jungle fowl, the ancestor to modern domestic chicken breeds, suggest the natural flock size may be on the order of 4 to 30 birds, too small, perhaps, for such rare events to happen with frequency. Though LPAI H5 and $\mathrm{H} 7$ avian influenza virus subtypes may demonstrably have both the replicative apparatus and a sufficient number of susceptible hosts in an intensive poultry production setting to acquire the mutations necessary to become highly pathogenic viruses, stochastic models do not explain what selective pressures might drive this evolution towards higher pathogenicity. However, there is a rich biological literature on the evolution of virulence from which to draw.

4a. Selection for Virulence: Evolutionary Theory. The conventional view on virulence, entrenched by Smith (1934) and popularized by Dubos (1965) and Burnet and White (1962), held that natural selection favored pathogen evolution towards benign co-existence with its host. This has been replaced with a nuanced view, supported empirically by studies on a variety of pathogens and parasites, that under certain circumstances, increased virulence may hold a selective advantage (Ebert 1995). Just as peaceful coexistence may be precluded in predator-prey systems, "predator-like" variants of a pathogen population may out-produce and out-transmit more benign pathogens (Ewald 1994). Frank and Jeffrey (2001) specifically used examples of virulent zoonotic pathogens to argue that newly established parasite-host relationships may be associated with virulence given the lack of efficient host-defense specialization.

Although there is no grand unified theory of the evolution of virulence, a number of proposals have been put forward (Schall 2002). The Transmission-Opportunity Hypothesis proposes that evolution towards virulence is favored in situations where pathogens have greater prospects for moving from host to host; otherwise, the host could die before transmission occurs (Gill and Mock 1985). The Transmission-Titer 
Hypothesis suggests that virulence requires high infectious loads, lest repeated pathogen population bottlenecks reduce overall fitness (Clarke et al. 1993). The Mobility Hypothesis suggests that host mobility-independent transmission favors virulence, as is seen in many vector and water-borne diseases (Ewald 1995). Likewise, the Sit-and-Wait Hypothesis predicts that the environmental durability of pathogens would correlate with virulence, as this would also reduce the fitness costs of disabling the host.Walther and Ewald (2004) found just such a correlation in human respiratory pathogens. The HostDemography Hypothesis asserts that selection would favor virulence in hosts with short lifespans to increase transmission probability (Ebert and Mangin 1997), and the Small Worlds-Diminishing Returns Hypothesis proposes that greater size and connectivity of the global host population may also allow for the evolution of virulence (Boots and Sasaki 1999). The industrial ecology of intensive poultry production arguably meets the criteria for all of these models of virulence selection.

These models are based largely on the "trade-off model" assumption that viral load (or more broadly, parasitemia) is both negatively correlatedwith host survival and positively correlated with probability of transmission. In cases in which the intensity of host exploitation-virulence-may facilitate transmission, such as in the fits of coughing and diarrhea characteristic of influenza and cholera, respectively, the model presumes that pathogens must strike a balance between intensity of infectivity and duration. Transmission may end when the host's life does. Natural selection is then thought to mediate this evolutionary process, choosing over time the virus with the optimum balance of lethality and contagion (Dimmock 2001). Though this underlying assumption between virulence and reproductive capacity is intuitively reasonable, it has been rarely studied (Mackinnon and Read 1999) and exceptions abound (Messenger et al. 1999).

While it is clear that virulence in the case of HPAI viruses may be negatively correlated with host survival, the selective advantage of HPAI over LPAI transmission is presumed but has not been unequivocally demonstrated. Generally, horizontally transmitted (Messenger et al. 1999) pathogens with high clonal diversity (van Baalen and Sabelis 1995) may tend towards virulence. When such a pathogen passes into a new environment, species, or even host, it may start out with little pathogenicity, but then be selectively pressured into an "acute life strategy" to overwhelm host defenses (van Blerkom 2003). Such a selective advantage may exist for influenzavirus $A$ variants with the potential capacity ( $\mathrm{H} 5$ and $\mathrm{H} 7$ subtypes) to become virulent as long as they do not suffer a related comparable loss of fitness (Suarez 2000). Studies on avian influenza viruses have shown that greater virulence does not necessarily translate into greater transmissibility (Narayan et al. 1969; Alexander et al. 1978; Westbury et al. 1979; Westbury et al. 1981; Alexander et al. 1986), but each of the variably pathogenic viruses contrasted were different strains, making direct comparison impossible. The findings may also be difficult to extrapolate to an agricultural setting from the laboratory environment (i.e., tiled floors, no litter, twice-daily removal of dead and dying birds). In general, bird-to-bird transmission remains poorly understood and likely depends on a complex interaction among the virus strain, bird species, and environmental factors (Capua and Alexander 2006a).

4a(1). The host-demography hypothesis. The Host-Demography Hypothesis is consistent with the blame for HPAl emergence that has been placed in part on the rapid production cycles (Capua and Marangon 2003b) and high-density confinement (Suarez et al. 2003) of today's industrial poultry production system. In modern broiler chicken production, 20,000 to 30,000 day-old chicks are typically cycled to slaughter approximately every 45 days per house (Hugh-Jones et al. 1995). The regular depopulation and repopulation of broiler grow-out sheds provide a constant influx of susceptible hosts and, potentially, the selecting for virulent variants (as per the Host-Demography Hypothesis) that might otherwise be destroyed with their host at the slaughter plant.

4a(2). The small worlds-diminishing returns hypothesis. The Small Worlds-Diminishing Returns Hypothesis suggests that small, isolated host populations might disfavor virulence, which is clearly not the case in intensive production systems that not only confine large host populations, but may be linked through global networks of live trade that deal in hundreds of millions of chickens a year (FAO 2007b).

4a(3). The Sit-and-Wait hypothesis. Pathogen buildup may result from stocking density-associated deteriorations in air and litter quality. A 20,000-bird broiler grow-out shed has been estimated to produce more than one ton of droppings daily (Wilson 1966) and may not be cleaned out between flocks, exposing 
additional populations of poultry to a variety of pathogens (Zavala 1998). During the 1983-1985 HPAI H5N2 outbreak in Pennsylvania, virus was detectable in wet manure after 105 days under field conditions (Fitchner 1987), with each gram of feces containing up to 107 potentially infectious doses (Utterback 1984). The increased relative humidity associated with high-density stocking, as well as the evaporative cooling misting used in many Asian commercial poultry production facilities to reduce heat stress (Delgado and Narrod 2002), may enhance the environmental stability of the virus, particularly as HPAl viruses seem to rapidly inactivate as feces dry (Shortridge et al. 1998). Birds reared indoors may be more susceptible to disease due to diminished air quality and the accumulation of pathogens in a restricted environment, as well as the relative lack of sunlight exposure (Ritchie 1995). Songserm et al. (2006) demonstrated that H5N1 in chicken feces was completely inactivated within 30 minutes in direct sunlight. First made possible by the discovery of vitamin $D$ in the 1920 s, total indoor confinement enables producers to raise flocks year-round and modify lighting schedules to optimize feed conversion (Russell and Fairchild 2005), but may have epizootic and zoonotic consequences.

Lack of adequate ventilation and sunlight typical to intensive confinement production systems may directly facilitate the spread of influenza. Decreased air exchange is strongly associated with increased influenza infection rates in laboratory animals (Bridges et al. 2003), just as crowded, enclosed spaces may increase influenza transmission in humans (Drexler 2002). A study performed during the 1957-1958 pandemic found significantly lower influenza rates in patients living in rooms with, versus without, UV antiTB lights, suggestive of the influence of direct sunlight in influenza inactivation (Bridges et al. 2003).

According to the Sit-and-Wait Hypothesis, the potential for enhanced environmental stability in intensive poultry production systems may also intensify selection for virulence. In a persistently contaminated environment, such as a commercial broiler chicken grow-out shed, even if an animal rapidly succumbs to infection, the virus may survive for weeks in wet manure and thereby not suffer a concomitant total loss in fitness. Should a virus mutant become highly pathogenic in a more extensive, sunlit, ventilated setting, then genetic virulence determinants may dead-end with the host.

4a(4). The transmission-opportunity hypothesis. Industrial poultry populations are not only large, but dense, a coupling that may directly and indirectly affect the evolution of HPAl viruses. Directly, high stocking densities may increase both the number and frequency of infectious contacts, allowing the virus to cycle rapidly from host to host. Indeed, since some of the first characterization studies were published in 1942, size and/or stocking density of chicken flocks have reportedly been associated with increased contact transmission of HPAl. For example, when a chicken intramuscularly infected with an HPAl turkey virus (turkey/Ontario/7732/66) was placed in a room with five chickens and two turkeys, the experimentally infected chicken and two turkeys died, but none of the five chickens fell ill. The same experiment was repeated, but with 13 chickens. In this higher chicken-density scenario, five of the contact chickens became sick and three died (Narayan et al. 1969). Active surveillance for H9N2 in broiler chicken breeder flocks in Jordan more recently found flock size positively associated with the percentage of sero-positive chickens detected in each sampled flock (Al-Natour and Abo-Shehada 2005).

Indirectly, overcrowding has been shown to impair adaptive immunity in chickens, presumably through the mechanism of immunosuppressive stress (El-Lethey et al. 2003), which may result from a frustration of natural behaviors at unnatural stocking densities (Dawkins and Hardie 1989). Naturally, chickens develop complex social hierarchies, yet this pecking order may also be disrupted in intensive production systems and induce a social stress that has been shown for nearly 30 years to weaken resistance to viral infection (Craig 1978) more a multitude of other disease challenges (Siegel 1983).

Over time, chickens overcrowded in confinement develop an enlargement of their adrenal glands and a regression in lymphoid tissue (Siegel 1983), which suggests a mechanism for crowding-induced disease susceptibility - a metabolic trade-off in which energies invested in host defense may be diverted by the stress response (Puvadolpirod and Thaxton 2000). Thiswas reportedly one reason the European Union banned the use of the conventional battery cage for egg-laying hens, effective 2012 (SVC 1996). Diminished immune function may also mean diminished protective response to vaccination (Witter 1998), which might otherwise abolish influenza virus shedding in some birds (Middleton et al. 2007). The temperature of poultry houses has been speculatively tied to both avian and human airway exaptation. It 
may not just be the heat, but the humidity. The putrefaction of wet fecal material may generate ammonia (Cole et al. 1999), which may predispose birds to respiratory infection either directly, by damaging the birds' airways (Cooper et al. 1996) or interfering with leukocyte function (NTF 1995), or indirectly, via the stress of cutaneous ammonia burns such as breast blisters, hock burns, and footpad dermatitis (Jones et al. 2005). A multinational study of dozens of commercial farms found that ammonia levels significantly increased corticosteroid levels, a potent stress-related immune depressant (Van der Sluis 2005). Although airborne aerosol spread of H5N1 remains relatively inefficient even among chickens (Sims et al. 2003), the airway damage and immunosuppression associated with ammonia levels that can accompany high stocking densities may speculatively facilitate avian influenza virus pneumotropism (Hafez 2000).

Increased stocking density also leads to high concentrations of aerial pollutants. Poultry houses can average more than an estimated one million aerial bacteria in every cubic meter (Hafez 2000), which may overwhelm lung clearance mechanisms. Madelin and Wathes (1989) demonstrated that exposing chickens to a normally nonpathogenic strain of $E$. coli in an environment clouded with dust or ammonia could result in disease. H5N1 transmission experiments reveal that chicken-to-chicken spread of H5N1 is predominantly via the fecal-oral route, rather than in airborne respiratory droplets, but the levels of fecal dust present inside commercial poultry houses (Cole et al. 1999) may have the potential to add a "fecaltracheal" route as well.

This Transmission-Opportunity Hypothesis posits that virulence development is favored in situations where pathogens have greater prospects for host-to-host movement. This could result from this multiplicity of conditions found in industrial poultry production settings, including enhanced virus environmental stability, the numerous routes of exposure, and increased vulnerability due to stressinduced immunosuppression or compromised respiratory status. All of these factors together may result in the efficient circulation of LPAI viruses in intensive systems and therefore, according to the TransmissionOpportunity Hypothesis, explainwhy certain LPAI virusesmay tend to transform into HPAI viruses.

4a(5). The transmission-titer hypothesis. Serial transmission experiments have shown that virulence can be rapidly increased in some pathogens, presumably since they no longer pay a fitness cost for killing their hosts since transmission is guaranteed (Ebert 1998). If the infectious load in each transfer is too low, however, pathogen virulence may decline (Clarke et al. 1993). Brown et al. (2001) showed that the intranasal serial passaging of human influenza virus-infected murine lung tissue in mice could change a seasonal human influenza virus, normally avirulent in mice, into a mouse-adapted strain capable of causing rapidly fatal pneumonia in exposed animals via a 12-mutation difference between pre- and postpassaging avirulant and lethal strains. The virus titer of the transferred innocula was considered key to this escalation of virulence. Since RNA virus replication may be so error-prone there may be pseudospecies members capable of causing heightened host exploitation and, therefore, presumably an increase in virulence should they transfer, but such isolates may be rare. A significant fraction of the mutant swarm may in fact be dysfunctional. As such, if only small titers of virus are transferred from one host to another, the virus might even lose potency (Domingo et al. 1997). The greater the number of viruses passaged, the greater the pool from which natural selection may select. The evolution of extremely rare though potentially adaptive traits such as hypervirulence, as may be seen in viruses like $\mathrm{H} 5 \mathrm{~N} 1$, may then speculatively require passaging of massive viral loads present in certain laboratory circumstances or, perhaps, in certain industrial poultry settings.

These various hypotheses may contribute to our understanding as to why an LPAl to HPAl virus transformation has never been known to arise in an outdoor flock of chickens or turkeys, even though penned turkey flock sizes may reach 100,000 birds (Halvorson et al. 1985). Though poultry raised outdoors are demonstrably more vulnerable to exposure to LPAI viruses carried by wild birds in certain regions (Hall 2004), should an LPAI outbreak occur, the sunlight and fresh air presumably limit the transmission efficiency required for the acquisition of the mutations associated with high pathogenicity.

Because influenza viruses are rapidly inactivated by sunlight and desiccation, their ability to spread efficiently from one chicken to the next outside in the open air is relatively limited. Therefore, in extensive systems, where bird densities and transmission titers may be lower, high host mortality may be disadvantageous for spread and even lead to loss in virus virulence, whereas conditions in intensive 
systems may favor the evolution, preservation, and rapid transmission of highly virulent strains (GauthierClerc et al. 2007). Two HPAl outbreaks, both in South Africa, have been recorded in birds in the open air-among wild sea-birds in 1961 (Becker 1966) and two outdoor ostrich farms in 2004 (Sabirovic 2004)_-and there have been rare sporadic outbreaks dating back more than 125 years (Perroncito 1878). These, however, seem to be exceptions to the rule (Stegeman 2003).

4a(6). The mobility hypothesis. Wild ducks staging for migration may also be intensely crowded and exposed to high titers of efficiently transmitted fecal $\mathrm{H} 5$ or $\mathrm{H} 7$ influenza subtype LPAI viruses that are even likelier to remain environmentally resilient in cold, high-latitude lakes. The Mobility Hypothesis may explain why HPAl viruses rarely arise in this setting. Given the stresses of migratory flight, sickened birds, and any viruses they might carry, may not travel far (Gylfe et al. 2000). When persistence of infection is dependent on host mobility, the range of clinicopathological manifestations is presumably constrained by the necessity of keeping the host healthy enough, long enough, to sufficiently spread to others, especially for such short-lived infections such as influenza. When stressed animals are overcrowded "trachea-totrachea" in unhygienic and confining environs where they may be unable to escape infectious contacts, no such natural biological checks on virulence may apply. Indeed, this same model of virulence that has been applied to HPAl evolution has also been used to explain the emergence of the pandemic virus of 1918.

4b. Selection for Virulence: 1918. To test the robustness of the hypothesis that intensive poultry production conditions facilitate the emergence of virulent avian influenza viruses, one might ask if such conditions have facilitated the emergence of such viruses in the past.

First proposed by Ewald (1994) and later expanded by Byerly (2005), the suggestion is that the 1918 virus built up its virulence in the transport ships, trains, camps, and trenches of World War I, as millions of young men were forced together in close quarters where there was no escaping a sick comrade. The crowded, stressful, unhygienic conditions at the Western Front are argued to have favored the evolution of a "predator-like" virus that otherwise may have killed too rapidly to spread with peak efficiency under normal circumstances (McGirk et al. 2005). Oxford et al. (2002) took the argument a step further by producing photographic evidence of goose and chicken markets and pig farms installed at the Great City British Army Camp of Etaples, at which 100,000 soldiers were crowded into tents and temporary barracks on any given day with continuous, bidirectional circulation of wounded survivors and susceptible recruits. In the absence of air travel, the virus may have "simmered" for months or years in such a camp, relying on the demobilization in the fall of 1918 for rapid global dispersal (Oxford 2001).

Ewald (1994) uses the Mobility Hypothesis to argue the point, suggesting that because mobilityindependent pathogen dispersal mechanisms may incur less of a liability from host infirmity, there may have been little Darwinian limit to virulence under such conditions. The proposed key to the evolution of high-pathogenicity pathogens, then, was crowding sufficient to allow for transmission from immobilized hosts to susceptible hosts packed together shoulder-to-shoulder, or, potentially, beak-to-beak (Ewald 1996). In effect, what Ewald and Byerly seem to be arguing is that in 1918, the soldiers were the poultry.

Byerly (2005) emphasized that the uniqueness of the conditions on the Western Front meant a 1918scale pandemic was unlikely to ever be repeated. This may, speculatively, account for the approximate 80-year lag between the last time a wholly avian flu virus appears to have produced human mortality1918-and when H5N1 acquired human pathogenicity in 1997, but these same "trench-warfare" conditions can be thought to exist at present in many respects in industrial poultry production. Given the seeming idiosyncratic similarity of certain avian influenza virus binding characteristics between human and gallinaceous poultry, concern may continue to surround the public health implications of the high poultry densities, increased stressors, and low sanitary standards that may exist in intensive production, potentially favoring the selection, maintenance, and transmission of virulent subtypes of HPAI.

4c. Selection for Virulence: Other Poultry Viruses. Evolutionary theory turned biologists from taxonomists into theorists. The elegance of evolutionary biology is that it produces testable predictions, traditionally more characteristic of the "hard" sciences like physics. For any scientific principle to be useful, it must be generalizable at least to a certain extent so as to serve a predictive function. If the conditions found in 
many industrial poultry production settings facilitate the emergence of virulence in avian influenza viruses, then might it not have the same effect on other poultry viruses? The robustness of the hypothesis, then, that intensive poultry production conditions are permissive or even facilitative of the development of virulence in viral pathogens can be assessed by looking to see if other poultry viruses fit the pattern.

Newcastle disease virus, another RNA virus now causing widespread epizootic outbreaks among poultry, shows striking similarities to avian influenza virus in that it also exists in both low pathogenicity (lentogenic) and highly pathogenic (velogenic) forms differentially based on the presence of a multibasic sequence at the fusogenic protein cleavage site (Seal et al. 2000). Furthermore, Newcastle disease virus may circulate as avirulent strains in wild waterfowl and acquire pathogenicity in intensive commercial poultry settings (Takakuwa et al. 1998).

Infectious bursal disease (IBD) is a poultry immunodeficiency disease caused by an RNA virus that infects B-cells, analogous to HIV's tropism for T-helper cells, leaving surviving birds with impaired antibody production, resultant poor vaccinal immunity, and susceptibility to a wide variety of viral, bacterial, and parasitic diseases (Bumstead 2003). Beginning in the 1980s, an escalation of respiratory disease in U.S. poultrywas traced to a hypervirulent "Delaware variant" strain of IBD virus that newly emerged from likely the most concentrated poultry production area in the world (Silbergeld 2006), the Delmarva peninsula (Cereno 2007). The subsequent global spread of Delaware variant IBD virus from the U.S. Eastern Seaboard was blamed on intensive poultry production and trade (Shane 2003). Evidence of IBD virus even in Antarctic Emperor penguins has been considered an example of industrial animal agriculture's "pathogen pollution" of the environment (Daszak et al. 2003).

There has also been a dramatic increase in the virulence of another poultry affliction in recent decades, Marek's disease (MD) (Boyd 2001), caused by an immunosuppressive oncogenic alphaherpesvirus (Schat and Davies 2000). Nair (2005) implicates intensive poultry production in the transformation of the virus from mMDV (mild Marek's disease virus) to vMDV (virulent Marek's disease virus), and subsequent evolution to vVMDVand now vv+MDV.

Newcastle disease virus (Evans 1955), IBD virus (Pedersden et al. 1990), and MDV (Laurent et al. 2001) have little, if any, direct zoonotic potential. However, poultry immunodeficiency viruses such as IBD virus, MDV, and chicken infectious anemia virus (CIAV), which is also associated with intensive production (Miller and Schat 2004), may increase zoonotic risk indirectly through an immunosuppressive effect on gallenaceous hosts of zoonoses. A speculative connection was drawn between IBD outbreaks in Hong Kong chickens in 1996 and the emergence of H5N1 in 1997 (NN 2004), but no evidence has been submitted to support this supposition. Ironically, one reason given for not cleaning and disinfecting poultry houses between flocks is that early infection with environmentally persistent viruses like CIAV may lead to a clearance of infectivity before breeding chickens are able to potentially pass the virus to progeny (Fussell 1998).

4d. Poultry Intensification and Selection for Virulence: Implications. Escalations in commercial poultry production similar to those in China started taking place in the 1980s throughout Southeast Asia (FAO 2007b), considered the hub of the global Livestock Revolution (Delgado and Narrod 2002). Around the world, the majority of pork and poultry is now produced in large-scale, industrial units (De Haan et al. 1997) with further intensification predicted for the foreseeable future (UNDP 2003).

Kennedy Shortridge, co-originator of the hypothesis that southern China is an "epicenter" of pandemic emergence (Shortridge and Stuart-Harris 1982), describes poultry intensification as the "crux" of the problem (Greger 2006), speculating that the intensification of the poultry sector worldwide may facilitate pandemic emergence elsewhere (Shortridge et al. 2003), as the world's estimated 50 billion-strong influenzavirus A reservoir of migrating geese, ducks, and swans is increasingly being supplemented with an additional 45 billion farmed chickens every year (FAO 2007b).

Over the last six decades, the industrial poultry sector has streamlined husbandry systems to maintain nearly complete management over birds' lives and movements. Yet, despite this and the battery of vaccines, antimicrobials, and dewormers that may be employed, the poultry industry remains beset by 
epizootic disease, understood to be a consequence of intensification (Shane 2003). Even with great financial disease-associated loss, intensive production is still considered by industry to make good business sense compared to the low-output extensive systems that have been increasingly replaced around the world (Pond and Pond 2000). Where there is no direct or indirect zoonosis-associated risk, it may be considered industry's prerogative to profitably employ least-cost production methods of their choosing, but given the emergence ofH5N1and a growing understanding of the zoonotic risks associated with the intensive poultry production model, it may instead be argued that such significant disease losses can no longer be factored into the bottom-line. External regulation may be required to prevent further industry externalization of costs associated with zoonotic poultry disease.

\section{CONCLUSION: ONE MEDICINE}

The term zoonosis was coined by Rudolph Virchow in 1855 during his studies of Trichinella, which reportedly helped convince him of the importance of the concept of "one medicine," in which human and animal health are inextricably linked (Brown 2004). Given the modern Renaissance in emerging zoonotic disease not seen, perhaps, since the Neolithic Revolution, never before may this notion have been more compelling than it is today.

A consistent theme that arises across studies of emerging zoonoses is the anthropogenic nature of identified risk factors. Typically and simplistically referred to as "ecological changes," upon closer look, humans tend to be the ones effecting the change. Homo sapiens has dramatically altered the ecological landscape in which other species and their pathogens must function. Along with human culpability, though, comes hope: If changes in human activity can cause new diseases, then changes in human activity may prevent them in the future.

The recognition that nearly three-quarters of emerging diseases may have arisen from the animal kingdom highlights how artificial the separation is between the human species and the rest of the natural world. Our growing understanding of the links between environmental disturbance and disease may require a $21^{\text {st }}$ century update to Virchow's venerable lesson, an extension of the one medicine concept to include not only animal and human health, but planetary health as well.

\section{REFERENCES}

Aarestrup, F.M., Jorsal, S.E., and Jensen, N.E. 1998. Serological characterization and antimicrobial susceptibility of Streptococcus suis isolates from diagnostic samples in Denmark during 1995 and 1996. Vet. Microbiol. 60, 59-66.

Abolnik, C., Bisschop, S., Gerdes,T., Olivier, A., and Horner, R. 2007. Outbreaks of avian influenza H6N2 viruses in chickens arose by a reassortment of H6N8 and H9N2 ostrich viruses. Virus Genes 34, 37-45.

Acar, J.F., and Moulin, G. 2006. Antimicrobial resistance at farm level. Rev. Sci. Tech. Off. Int. Epiz. 25, 775-792.

Achong, B.G., Mansell, P.W.A., Epstein, M.A., and Clifford, P. 1971.Anunusual virus in cultures from a human nasopharyngeal carcinoma. J. Natl. Cancer Inst. 46, 299-307.

Achtman, M., Zurth, K., Morelli, G., Torrea, G., Guiyoule, A., and Carniel, E. 1999. Yersinia pestis, the cause of plague, is a recently emerged clone of Yersinia pseudotuberculosis. Proc. Natl. Acad. Sci. USA 96, 14043-14048.

Adams, M.W., Ellingboe, A.H., and Rossman, E.C. 1971. Biological uniformity and disease epidemics. BioScience 21, 1067-1070.

Adams, M., and Motarjemi, Y. 1999. Basic Food Safety for Health Workers. Geneva, Switzerland: World Health Organization Press.

Al-Natour, M.Q., and Abo-Shehada, M.N. 2005. Sero-prevalence of avian influenza among broilerbreeder flocks in Jordan. Prev. Vet. Med. 70, 45-50.

Alderman, D.J., and Hastings, T.S. 1998. Antibiotic use in aquaculture: Development of antibiotic resistance potential for consumer health risks. Int. J. Food Sci. Tech. 33, 139-155.

Alexander, D.J. 2007. An overview of the epidemiology of avian influenza. Vaccine 25, 5637-5644. 
Alexander, D.J., Allan,W.H., Parsons, D., and Parsons, G. 1978. The pathogenicity of four avian influenza viruses for chickens, turkeys and ducks. Res. Vet. Sci. 24, 242-247.

Alexander, D.J., Capua, I., and Brown, I.H. 2003. Avian influenza viruses and influenza in humans. In Proceedings of the Frontis Workshop on Avian Influenza: Prevention and Control, eds. R.S. Schrijver and Koch, G., 1-12.

Wageningen, The Netherlands. Alexander, D.J., Parson, S.G., and Manvell, R.J. 1986. Experimental assessment of the pathogenicity of eight avian influenza A viruses of $\mathrm{H} 5$ subtype for chickens, turkeys, ducks and quail. Avian Pathol. 15, 647-662.

Allan, B.F., Keesing, F., and Ostfeld, R.S. 2003. Effect of forest fragmentation on Lyme disease risk. Conserv. Biol. 17, 267-272.

Allan, J.S., Broussard, S.R., Michaels, M.G., Starzl, T.E., Leighton, K.L., Whitehead, E.M., Comuzzie, A.G., Lanford, R.E., Leland, M.M., Switzer, W.M., and Heneine, W. 1998. Amplification of simian retroviral sequences from human recipients of baboon liver transplants. AIDS Res. Hum. Retroviruses. 14, 821-824.

Amass, S.F., Clark, L.K., Knox, K.,Wu, C.C., and Hill, M.A. 1996. Streptococcus suis colonization of piglets during parturition. J. Swine Health Prod. 4, 269-272.

Anand, C.M., Finlayson, M.C., Garson, J.Z., and Larson, M.L. 1980. An institutional outbreak of salmonellosis due to lactose-fermenting Salmonella Newport. Am. J. Clin. Path. 74, 657-660.

Anderson, A.D., McClellan, J., Rossiter, S., and Angulo, F.J. 2003. Public health consequences of use of antimicrobial agents in agriculture. In The resistance phenomenon in microbes and infectious disease vectors: Implications for human health and strategies for containment: Workshop summary, 231-243.

Washington, D.C.: National Academies Press. Anderson, R.M., Donnelly, C.A., Ferguson, N.M.,Woolhouse, M.E.,, Udy, H.J., MaWhinney, S., Dunstan, S.P., Southwood, T.R.,Wilesmith, J.W., Ryan, J.B., Hoinville, L.J., Hillerton, J.E., Austin, A.R., and Wells, G.A. 1996. Transmission dynamics and epidemiology of BSE in British cattle. Nature 382, 779-788.

Anderson, R.M., Fraser, C., Ghani, A.C., Donnelly, C.A., Riley, S., Ferguson, N.M., Leung, G.M., Lam, T.H., and Hedley, A.J. 2004. Epidemiology, transmission dynamics and control of SARS: The 2002-2003 epidemic. Philos. Trans. R. Soc. Lond. B. Biol. Sci. 359, 1091-1105.

Anderson, R.M., and May, R.M. 1986. The invasion, persistence and spread of infectious diseases within animal and plant communities. Philos. Trans. R. Soc. Lond. B. Biol. Sci. 314, 533-570.

Andr'e, F., and Tuyttens, M. 2005. The importance of straw for pig and cattle welfare: a review. Appl. Anim. Behav. Sci. 92, 261-282.

Andreasen, C.B., Spickler, A.R., and Jones, B.E. 2005. Swedish antimicrobial regulations and their impact on food animal production. J. Am. Vet. Med. Assoc. 227, 41-45.

Angulo, F.J., and Griffin, P.M. 2000. Changes in antimicrobial resistance in Salmonella enterica serovar typhimurium. Emerg. Infect. Dis. 6, 436-438.

Annan, K. 2001. Remarks to the United States Chamber of Commerce. Washington, D.C., June 1.

Arabi, Y., Gomersall, C.D., Ahmed, Q.A., Boynton, B.R., and Memish, Z.A. 2007. The critically ill avian influenza A (H5N1) patient. Crit. Care Med. 35, 1397-1403.

Arends, J.P., Harwig, N., Rudolphy, M., and Zanen, H.C. 1984. Carrier rate of Streptococcus suis capsular type 2 in palatine tonsils of slaughtered pigs. J. Clin. Microbiol. 20, 945-947.

Arends, J.P., and Zanen, H.C. 1988. Meningitis caused by Streptococcus suis in humans. Rev. Infect. Dis. 10, 131-137.

Arlet, G., Barrett,T.J., Butaye, P., Cloeckaert, A., Mulvey, M.R., and White, D.G. 2006. Salmonella resistant to extended-spectrum cephalosporins: Prevalence and epidemiology. Microb. Infect. 8, 1945-1954.

Armelagos, G.J., Brown, P.J., and Turner, B. 2005. Evolutionary, historical and political economic perspectives on health and disease. Soc. Sci. Med. 61, 755-765.

Armstrong, G.L., Conn, L.A., and Pinner, R.W. 1999. Trends in infectious disease mortality in the United States during the 20th century. JAMA 281, 61-66.

Arthur, T.M., Bosilevac, J.M., Brichta-Harhay, D.M., Guerini, M.N., Kalchayanand, N., Shackelford, S.D., Wheeler, T.L., and Koohmaraie, M. 2007. Transportation and lairage environment effects on prevalence, numbers, and diversity of Escherichia coli $\mathrm{O} 157: \mathrm{H} 7$ on hides and carcasses of beef cattle at processing. J. Food Prot. 70, 280-286. 
Atabay, H.I., Corry, J.E.L., and On, S.L.W. 1998. Identification of unusual Campylobacter-like isolates from poultry products as Helicobacter pullorum. J. Appl. Microbiol. 84, 1017-1024.

Atwill, E.R., Johnson, E.M., and Pereira, M.G. 1999. Association of herd composition, stocking rate, and duration of calving season with fecal shedding of Cryptosporidium parvum oocysts in beef herds. J. Am. Vet. Med. Assoc. 215, 1833-1838.

Ayele, W.Y., Neill, S.D., Zinsstag, J., Weiss, M.G., and Pavlik, I. 2004. Bovine tuberculosis: An old disease but a new threat to Africa. Int. J. Tuberc. Lung Dis. 8, 924-937.

Badrane, H., and Tordo, N. 2001. Host switching in Lyssavirus history from the Chiroptera to the Carnivora orders. J. Virol. 75, 8096-8104.

Bailey, M.T., Karaszewski, J.W., Lubach, G.R., Coe, C.L., and Lyte, M. 1999. In vivo adaptation of attenuated Salmonella typhimurium results in increased growth upon exposure to norepinephrine. Physiol. Behav. 67, 359-364.

Bankowski, R.A. 1981a. Introduction and objectives of the symposium. In Proceedings of the First International Symposium on Avian Influenza, ed. Bankowski, R.A., vii-xiv, April 22-24, Beltsville, MD.

Banks, J., Speidel, E.S., Moore, E., Plowright, L., Piccirillo, A., Capua, I., Cordioli, P., Fioretti, A., and Alexander, D.J. 2001. Changes in the haemagglutinin and the neuraminidase genes prior to the emergence of highly pathogenic H7N1 avian influenza viruses in Italy. Arch. Virol. 146, 963-973.

Barham, A.R., Barham, B.L., Johnson, A.K., Allen, D.M., Blanton, J.R., Jr., and Miller, M.F. 2002. Effects of the transportation of beef cattle from the feedyard to the packing plant on prevalence levels of Escherichia coli 0157 and Salmonella spp. J. Food Prot. 65, 280-283.

Barnes, H.J.,Vaillancourt, J.P., and Gross,W.B. 2003. Colibacillosis. In Diseases of poultry, ed. Saif, Y.M., 631-652. Ames, IA: lowa State University Press.

Barnes, R. 2002. The bushmeat boom and bust in west and central Africa. Oryx 36, 236-242.

Battaglia, R.A. 2001. Handbook of Livestock Management, 3rd ed.Weimar, TX: Culinary and Hospitality Industry Publications Services.

Baumler, A.J., Hargis, B.M., and Tsolis, R.M. 2000. Tracing the origins of Salmonella outbreaks. Science $287,50-52$.

Bean, C., and Zhang, J. 2005. China livestock and products semi-annual. U.S. Department of Agriculture Foreign Agricultural Service.

Beare, A.S., and Webster, R.G. 1991. Replication of avian influenza viruses in humans. Arch. Virol. 119, $37-42$.

Becker, W.B. 1966. The isolation and classification of tern virus: Influenza A/tern/South Africa/1961. J. Hyg. 64, 309-320.

Beers, M.H., and Berkow, R., eds. 2005. Bacterial infections. In The Merck manual, 17th ed. Rahway, N.J.: The Merck Publishing Group.

Beigel, J.H., Farrar, J., Han, A.M., Hayden, F.G., Hyer, R., de Jong, M.D., Lochindarat, S., Nguyen, T.K., Nguyen, T.H., Tran, T.H., Nicoll, A., Touch, S., and Yuen, K.Y. Writing Committee of the World Health Organization (WHO) Consultation on Human Influenza A/H5. 2005. Avian influenza A (H5N1) infection in humans. N. Engl. J. Med. 353, 1374-1385.

Belay, T., Aviles, H., Vance, M., Fountain, K., and Sonnenfeld, G. 2003. Catecholamines and in vitro growth of pathogenic bacteria: Enhancement of growth varies greatly among bacterial species. Life Sci. 73, 1527-1535.

Bell, D., Roberton, S., and Hunter, P.R. 2004. Animal origins of SARS coronavirus: Possible links with the international trade in small carnivores. Philos. Trans. R. Soc. Lond. B. Biol. Sci. 359, 1107-1114.

Beniwal, M., Kumar, A., Kar, P., Jilani, N., and Sharma, J.B. 2003. Prevalence and severity of acute viral hepatitis and fulminant hepatitis during pregnancy: A prospective study from north India. Indian $\mathrm{J}$. Med. Microbiol. 21, 184-185.

Bennett, E.L., and Rao, M. 2002. Wild meat consumption in Asian tropical forest countries: Is this a glimpse of the future for Africa? In Links between biodiversity, conservation, livelihoods and food security: The sustainable use of wild species for meat, eds. Mainka, S. and Trivedi, M., 39-44. Gland, Switzerland: The World Conservation Union (IUCN).

Beran, G.W., and Steele, J.H. 1994. Handbook of Zoonoses. London, UK: CRC Press.

Berge, A.C., Lindeque, P., Moore, D.A., and Sischo,W.M. 2005. A clinical trial evaluating prophylactic and therapeutic antibiotic use on health and performance of preweaned calves. J. Dairy Sci. 88, $2166-2177$. 
Berge, A.C., Moore, D.A., and Sischo, W.M. 2006. Field trial evaluating the influence of prophylactic and therapeutic antimicrobial administration on antimicrobial resistance of fecal Escherichia coli in dairy calves. Appl. Environ. Microbiol. 72, 3872-3878.

Berthelot-Herault, F., Gottschalk, M., Labbe, A., Cariolet, R., and Kobisch, M. 2001. Experimental airborne transmission of Streptococcus suis capsular type 2 in pigs. Vet. Microbiol. 82, 69-80.

Bille, J. 1990. Epidemiology of human listeriosis in Europe, with special reference to the Swiss outbreak. In Foodborne listeriosis, eds. Miller, A.J., Smith, J.L., and Somkuti, G.A., 71-74. New York, NY: Elsevier.

Blattner, W.A. 1989. Human T-lymphotropic viruses and diseases of long latency. Ann. Intern. Med. 111, 4-6.

Boesch, C. 1994. Cooperative hunting in wild chimpanzees. Anim. Behav. 48, 653-687.

Bolton, L.F., Kelly, L.C., Lee, M.D., Fedorka-Cray, P.I., and Maurer, H. 1999. Detection of multidrugresistant Salmonella enterica serotype typhimurium DT104 based on a gene which confers cross-resistance to florfenicol and chloramphenicol. J. Clin. Microbiol. 37, 1348-1351.

Boots, M., and Sasaki, A. 1999. 'Small worlds' and the evolution of virulence: Infection occurs locally and at a distance. Proc. Biol. Sci. 266, 1933-1938.

Bosch, F.X., Orlich, M., Klenk, H.D., and Rott, R. 1979. The structure of the hemagglutinin, a determinant for the pathogenicity of influenza viruses. Virology 95, 197-207.

Bosman, A., Meijer, A., and Koopmans, M. 2005. Final analysis of Netherlands avian influenza outbreaks reveals much higher levels of transmission to humans than previously thought. Euro. Surveill. 10, E050106.2.

Bourry, A., and Poutrel, B. 1996. Bovine mastitis caused by Listeria monocytogenes: Kinetics of antibody responses in serum and milk after experimental infection. J. Dairy Sci. 79, 2189-2195.

Boxall, E., Herborn, A., Kochethu, G., Pratt, G., Adams, D., ljaz, S., and Teo, C.G. 2006. Transfusiontransmitted hepatitis $\mathrm{E}$ in a 'nonhyperendemic' country. Transfus. Med. 16, 79-83.

Boyd, W. 2001. Making meat: Science, technology, and American poultry production. Technol. Cult. 42, 631-664.

Brack, M. 1987. Agents Transmissible from Simians to Man. Berlin: Springer-Verlag.

Braden, C.R. 2006. Salmonella enterica serotype Enteritidis and eggs: A national epidemic in the United States. Clin. Infect. Dis. 43, 512-517.

Brahmbhatt, M. 2005. Avian and human pandemic influenza: Economic and social impacts. Geneva: WHO Headquarters, November 7-9.

Bridges, C.B., Kuehnert, M.J., and Hall, C.B. 2003. Transmission of influenza: Implications for control in health care settings. Clin. Infect. Dis. 37, 1094-1101.

Bright, R.A., Cho, D.S., Rowe, T., and Katz, J.M. 2003. Mechanisms of pathogenicity of influenza A (H5N1) viruses in mice. Avian Dis. 47, 1131-1134.

Brooks, J.I., Merks, H.W., Fournier, J., Boneva, R.S., and Sandstrom, P.A. 2007. Characterization of blood-borne transmission of simian foamy virus. Transfusion 47, 162-170.

Brosch, R., Gordon, S.V., Marmiesse, M., Brodin, P., Buchrieser, C., Eiglmeier, K., Garnier, T., Gutierrez, C., Hewinson, G., Kremer, K., Parsons, L.M., Pym, A.S., Samper, S., van Soolingen, D., and Cole, S.T. 2002. A new evolutionary scenario for the Mycobacterium tuberculosis complex. Proc. Natl. Acad. Sci. USA 99, 3684-3689.

Brown, C. 2000a. Emerging infectious diseases of animals: An overview. In Emerging diseases of animals, eds. C. Brownand Bolin, C., 1-12.Washington, D.C.: ASM Press.

Brown, C. 2004. Emerging zoonoses and pathogens of public health significance: An overview. Rev. Sci. Tech. Off. Int. Epiz. 23, 435-442.

Brown, E.G., Liu, H., Kit, L.C., Baird, S., and Nesrallah, M. 2001. Pattern of mutation in the genome of influenza $A$ virus on adaptation to increased virulence in the mouse lung: Identification of functional themes. Proc. Natl. Acad. Sci. USA 98, 6883-6888.

Brown, I.H. 2000b. The epidemiology and evolution of influenza viruses in pigs. Vet. Microbiol. 74, $29-46$.

Brown, P., Preece, M., Brandel, J.P., Sato, T., McShane, L., Zerr, I., Fletcher, A., Will, R.G., Pocchiari, M., Cashman, N.R., d'Aignaux, J.H., Cervenakova, L., Fradkin, J., Schonberger, L.B., and Collins, S.J. 2000. latrogenic Creutzfeldt-Jakob disease at the millennium. Neurology 55, 1075.

Brownstein, J.S., Skelly, D.K., Holford, T.R., and Fish, D. 2005. Forest fragmentation predicts local scale heterogeneity of Lyme disease risk. Oecologia 146, 469-475. 
Bruce, M.E., Will, R.G., Ironside, J.W., McConnell, I., Drummond, D., Suttie, A., McCardle, L., Chree, A., Hope, J., Birkett, C., Cousens, S., Fraser, H., and Bostock, C.J. 1997. Transmissions to mice indicate that 'new variant' CJD is caused by the BSE agent. Nature 389, 498-501.

Bumstead, N. 2003. Genetic resistance and transmission of avian bacteria and viruses. In Poultry genetics, breeding and biotechnology, eds. Muir, W.M. and Aggrey, S.E., 311-328. Oxfordshire, UK: CAB International.

Bunning, M., Jamaluddin, A., Cheang, H., Kitsutani, P., Muhendren, R., Olson, J., Karim, N., Field, H., Johara, M.Y., Sharihuddin, T., Choo Pow Yoon, R., Daniels, P., Ksiazek, T., and Nordin, M. 2000. Epidemiological traceback studies of the Nipah virus outbreak in pig farms in the Ipoh district of Malaysia, 1997-1999. Proceedings of the 16th International Pig Veterinary Society Congress, ed. CcaS McOrist, 551. Ocean Grove, USA: International Pig Veterinary Society Congress.

Bureau of Animal and Plant Health Inspection and Quarantine Council, Council of Agriculture (COA), Executive Yuan. 2003. H5N1 Avian influenza virus detected in smuggled birds from China. Republic of China.

Burkholder, J.M., and Glasgow, H.B. 1995. Insidious effects of toxic estuarine dinoflagellate on fish survival and human health. J. Toxicol. Environ. Health 46, 501-522.

Burnet, F.M. 1946. Virus as Organism. Cambridge, MA: Harvard University Press.

Burnet, F., and Clark, E. 1942. Influenza: A Survey of the Last 50 Years in the Light of Modern Work on the Virus of Epidemic Influenza. Melbourne, Australia: MacMillan.

Burnet, M., and White, D.O. 1962. Natural History of Infectious Disease, $4^{\text {th }}$ ed. Cambridge, UK: Cambridge University Press.

Burrell, A. 2002. Animal disease epidemics: Implications for production, policy and trade. Outlook on Agriculture 31, 151-160.

Busque, P., Higgins, R., Caya, F., and Quessy, S. 1997. Immunization of pigs against Streptococcus suis serotype 2 infection using a live avirulent strain. Can. J. Vet. Res. 61, 275-279.

Butler, D. 2006a. Bird-flu experts question advice on eating poultry. Nature 440, 850-851.

Butler, D. 2006b. Thai dogs carry bird-flu virus, but will they spread it? Nature 439, 773.

Butt, K.M., Smith, G.J., Chen, H., Zhang, L.J., Leung, Y.H., Xu, K.M., Lim, W.,Webster, R.G., Yuen, K.Y., Peiris, J.S., and Guan, Y. 2005. Human infection with an avian H9N2 influenza A virus in Hong Kong in 2003. J. Clin. Microbiol. 43, 5760-5767.

Byerly, C.R. 2005. Fever of War: The Influenza Epidemic in the U.S. Army During World War I, 94. New York, NY: New York University Press.

Byrd, J., Bailey, R.H., Wills, R., and Nisbet, D. 2007. Recovery of Campylobacter from commercial broiler hatchery trayliners. Poult. Sci. 86, 26-29.

Cameron, K.R., Birchall, S.M., and Moses, M.A. 1978. Isolation of foamy virus from patient with dialysis encephalopathy. Lancet 2, 796.

Cameron, K.R., Gregory, V., Banks, J., Brown, I.H., Alexander, D.J., Hay, A.J., and Lin, Y.P. 2000. H9N2 subtype influenza $A$ viruses in poultry in Pakistan are closely related to the H9N2 viruses responsible for human infection in Hong Kong. Virology 278, 36-41.

Cameron, R.D.A. 2000. A review of the industrialization of pig production worldwide with particular reference to the Asian region. Food and Agriculture Organization of the United Nations, Animal Production and Health Commission for Asia and the Pacific.

Campbell, C.H., Webster, R.G., and Breese, S.S. 1970. Fowl plague virus from man. J. Infect. Dis. 122, 513-516.

Campitelli, L., Fabiani, C., Puzelli, S., Fioretti, A., Foni, E., De Marco, A., Krauss, S.,Webster, R.G., and Donatelli, I. 2002.H3N2influenza viruses from domestic chickens in Italy: An increasing role for chickens in the ecology of influenza? J. Gen. Virol. 83, 413-420.

Campitelli, L., Mogavero, E., De Marco, M.A., Delogu, M., Puzelli, S., Frezza, F., Facchini, M., Chiapponi, C., Foni, E., Cordioli, P., Webby, R., Barigazzi, G., Webster, R.G., and Donatelli, I. 2004. Interspecies transmission of an H7N3 influenza virus from wild birds to intensively reared domestic poultry in Italy. Virology 323, 24-36.

Capua, I., and Alexander, D.J. 2004. Avian influenza: Recent developments. Avian Pathol. 33, 393-404.

Capua, I., and Alexander, D.J. 2006a. Avian influenza infection in birds: A moving target. Influenza and Other Respiratory Viruses 1, 11-18.

Capua, I., and Alexander, D.J. 2006b. The challenge of avian influenza to the veterinary community. Avian Pathol. 35, 189-205. 
Capua, I., and Marangon, S. 2000. The avian influenza epidemic in Italy, 1999-2000, A review. Avian Pathol. 29, 289-294.

Capua, I., and Marangon, S. 2003a. Currently available tools and strategies for emergency vaccination in case of avian influenza. In Proceedings of the FrontisWorkshop on Avian Influenza: Prevention and Control, eds. Schrijver, R.S., and Koch, G., October 13-15, The Netherlands, 59-74.

Capua, I., and Marangon, S. 2003b. The use of vaccination as an option for the control of avian influenza. In 71st General Session of the International Committee of the World Organization for Animal Health, May 18-23, Paris.

Capua, I., and Mutinelli, F. 2001. Mortality in Muscovy ducks (Cairina moschata) and domestic geese (Anser anser var. domestica) associated with natural infection with a highly pathogenic avian influenza virus of $\mathrm{H} 7 \mathrm{~N} 1$ subtype. Avian Pathol. 30, 179-183.

Capua, I., Mutinelli, F., Marangon, S., and Alexander, D.J. 2000. H7N1 avian influenza in Italy (19992000 ) in intensively reared chickens and turkeys. Avian Pathol. 29, 537-543.

Castrucci, M.R., Donatelli, I., Sidoli, L., Barigazzi, G., Kawaoka, Y., and Webster, R.G. 1993. Genetic reassortment between avian and human influenza A viruses in Italian pigs. Virology 193, 503506.

Cauthen, A.N., Swayne, D.E., Sekellick, M.J., Marcus, P.I., and Suarez, D.L. 2007. Amelioration of influenza virus pathogenesis in chickens attributed to the enhanced interferon-inducing capacity of a virus with a truncated NS1 gene. J. Virol. 81, 1838-1847.

Centers for Disease Control and Prevention (CDC). 1981. Pneumocystis pnuemonia-Los Angeles. MMWR Morb. Mortal Wkly. Rep. 20, 1-3.

Centers for Disease Control and Prevention (CDC). 1987. Epidemiologic notes and reports adult T-cell leukemia/lymphoma associated with human T-lymphotropic Virus Type I (HTLV-I) infectionNorth Carolina. MMWR Morb. Mortal Wkly. Rep. 36, 804-806, 812.

Centers for Disease Control and Prevention (CDC). 1999. Update: Outbreak of Nipah virus: Malaysia and Singapore. MMWR Morb. Mortal Wkly. Rep. 48, 335-337.

Centers for Disease Control and Prevention (CDC). 2002. Outbreak of multidrug-resistant Salmonella Newport-United States, January-April 2002. MMWR Morb. Mortal Wkly. Rep. 51, 545-548.

Centers for Disease Control and Prevention (CDC). 2005a. Escherichia coli O157:H7. National Center for Infectious Diseases Division of Bacterial and Mycotic Diseases, October 6.

Centers for Disease Control and Prevention (CDC). 2005b. Outbreaks in North America with transmission to humans.

Centers for Disease Control and Prevention (CDC). 2005c. Urinary tract infections. National Center for Infectious Diseases Division of Bacterial and Mycotic Diseases, October 25.

Centers for Disease Control and Prevention (CDC). 2006. Guidance and recommendations: Interim guidance for protection of persons involved in U.S. avian influenza outbreak disease control and eradication activities, January 14.

Cereno, T.N. 2007. Infectious bursal disease: Causative agent, diagnosis and prevention. Canadian Poultry Consultants Ltd.

Chalk, P. 2004. Hitting America's soft underbelly: The potential threat of deliberate biological attacks against the U.S. agricultural and food industry. Prepared for the Office of the Secretary of Defense. RAND National Defense Research Institute, <rand.org/pubs/monographs/2004/RAND MG135.pdf>.

Chapman, F.A. 1992. Farm-raised channel catfish. University of Florida, Institute of Food and Agricultural Sciences, Florida Cooperative Extension Service, Department of Fisheries and Aquatic Sciences, Circular 1052.

Chapman, L.E., Folks, T.M., Solomon, D.R., Patterson, A.P., Eggerman, T.E., and Noguchi, P.D. 1995. Xenotransplantation and xenogeneic infections. N. Engl. J. Med. 333, 1498-1550.

Charrel, R.N., and de Lamballerie, X. 2003. Arenaviruses other than Lassa virus. Antiviral Res. 57, 89100.

Check, E. 2004. Health concerns prompt US review of exotic-pet trade. Nature 427, 277.

Chen, G.W., Chang, S.C., Mok, C.K., Lo, Y.L., Kung, Y.N., Huang, J.H., Shih, Y.H., Wang, J.Y., Chiang, C., Chen, C.J., and Shih, S.R. 2006a. Genomic signatures of human versus avian influenza A viruses. Emerg. Infect. Dis. 12, 1353-1360. 
Chen, H., Deng, G., Li, Z., Tian, G., Li, Y., Jiao, P., Zhang, L., Liu, Z., Webster, R.G., and Yu, K. 2004. The evolution of H5N1 influenza viruses in ducks in southern China. Proc. Natl. Acad. Sci. USA 101, 10452-10457.

Chen, J., Powell, D., and Hu, W.S. 2006b. High frequency of genetic recombination is a common feature of primate lentivirus replication. J. Virol. 80, 9651-9658.

Chen, J.M., Chen, J.W., Dai, J.J., and Sun, Y.X. 2007. A survey of human cases of H5N1 avian influenza reported by theWHObefore June 2006 for infection control. Am. J. Infect. Control 35, 351-353.

Cheng, H.W., Singleton, P., and Muir, W.M. 2002. Social stress in laying hens: Differential dopamine and corticosterone responses after intermingling different genetic strains of chickens. Poult. Sci. 81, $1265-1272$.

Cheung, C.L., Vijaykrishna, D., Smith, G.J., Fan, X.H., Zhang, J.X., Bahl, J., Duan, L., Huang, K., Tai, H.,Wang, J., Poon, L.L., Peiris, J.S., Chen, H., and Guan, Y. 2007. Establishment of influenza A virus (H6N1) in minor poultry in southern China. J. Virol. Jul 25; [Epub ahead of print].

Chew-Lim, M., and Ng, C.Y. 1987. Recurrent viruses in a Singapore intensive pig farming estate. Ann. Acad. Med. Singapore 16, 651-654.

Childs, K.D., Simpson, C.A., Warren-Serna, W., Bellenger, G., Centrella, B., Bowling, R.A., Ruby, J., Stefanek, J., Vote, D.J., Choat, T., Scanga, J.A., J.N. Sofos, Smith, G.C., and Belk, K.E. 2006. Molecular characterization of Escherichia coli $\mathrm{O} 157: \mathrm{H} 7$ hide contamination routes: Feedlot to harvest. J. Food Prot. 69, 1240-1247.

Chin, P.S., Hoffmann, E., Webby, R., Webster, R.G., Guan, Y., Peiris, M., and Shortridge, K.F. 2002. Molecular evolution of $\mathrm{H} 6$ influenza viruses from poultry in Southeastern China: Prevalence of H6N1 influenza viruses possessing seven A/Hong Kong/156/97 (H5N1)-like genes in poultry. J. Virol. 76, 507-516.

Choi, Y.K., Lee, J.H., Erickson, G., Goyal, S.M., Joo, H.S., Webster, R.G., and Webby, R.J. 2004. H3N2 influenza virus transmission from swine to turkeys, United States. Emerg. Infect. Dis. 10, 21562160.

Choi, Y.K., Nguyen, T.D., Ozaki, H.,Webby, R.J., Puthavathana, P., Buranathal, C., Chaisingh, A., Auewarakul, P., Hanh, N.T., Ma, S.K., Hui, P.Y., Guan, Y., Peiris, J.S., and Webster, R.G. 2005a. Studies of H5N1 influenza virus infection of pigs by using viruses isolated in Vietnam and Thailand in 2004. J. Virol. 79, 10821-10825.

Choi, Y.K., Seo, S.H., Kim, J.A., Webby, R.J., and Webster, R.G. 2005b. Avian influenza viruses inKorean live poultry markets and their pathogenic potential. Virology 332, 529-537.

Chomel, B.B., Belotto, A., and Meslin, F.X. 2007. Wildlife, exotic pets, and emerging zoonoses. Emerg. Infect. Dis. 13, 6-11.

Chua, K.B., Chua, B.H., and Wang, C.W. 2002a. Anthropogenic deforestation, El Nirno and the emergence of Nipah virus in Malaysia. Malays. J. Pathol. 24, 15-21.

Chua, K.B., Lek, K.C., Hooi, P.S., Wee, K.F., Khong, J.H., Chua, B.H., Chan, Y.P., Lim, M.E., and Lam, S.K. 2002b. Isolation of Nipah virus from Malaysian Island flying-foxes. Microb. Infect. 4, 145151.

Chung,W.B., Lin,M.W., Chang,W.F., Hsu, M., and Yang, P.C. 1997. Persistence of porcine reproductive and respiratory syndrome virus in intensive farrow-to-finish pig herds. Can. J. Vet. Res. 61, 292298.

Claas, E.C., Kawaoka, Y., de Jong, J.C., Masurel, N., and Webster, R.G. 1994. Infection of children with avian-human reassortant influenza virus from pigs in Europe. Virology 20 4, 453-457.

Claas, E.C.J., and Osterhaus, A.D.M.E. 1998. New clues to the emergence of flu pandemics. Nature Med. 4, 1122-1123.

Claas, E.C., Osterhaus, A.D., van Beek, R., De Jong, J.C., Rimmelzwaan, G.F., Senne, D.A., Krauss, S., Shortridge, K.F., and Webster, R.G. 1998. Human influenza A H5N1 virus related to a highly pathogenic avian influenza virus. Lancet 351, 472-477.

Clague, B., Chamany, S., Burapat, C., Wannachaiwong, Y., Simmerman, J.M., S.F. Dowell, and Olsen, S.J. 2006. A household survey to assess the burden of influenza in rural Thailand. Southeast Asian J. Trop. Med. Public Health

37, 488-493.

Clark, G.M., Kaufmann, A.F., and Gangrosa, E.J. 1973. Epidemiology of an international outbreak of Salmonella agona. Lancet 2, 490-493. 
Clarke, D.K., Duarte, E.A., Moya, A., Elena, S.F., Domingo, E., and Holland, J. 1993. Genetic bottlenecks and population passages cause profound fitness differences in RNA viruses. Virology 67, 222228.

Clifton-Hadley, F.A. 1986. The epidemiology, diagnosis, treatment, and control of Streptococcis suis type 2 in pigs. Vet. Rec. 107: 40-41.

Clifton-Hadley, F.A., and Enright, M.R. 1984. Factors affecting the survival of Streptococcus suis type 2. Vet. Rec. 114, 584-586.

Cloeckaert, A., Sidi Boumedine, K., Flaujac, G., Imberechts, H., D'Hooghe, I., and Chaslus-Dancla, E. 2000. Occurrence of a Salmonella enterica Serovar Typhimurium DT104-like antibiotic resistance gene cluster including the floR gene in S. enterica Serovar Agona. Antimicrob. Agents Chemother. 44, 1359-1361.

Clough, J.D. 2004. Birds, viruses, and history: The current "genuine adventure." Clev. Clin. J. Med. 71, 270.

Cockburn, T.A. 1967. The evolution of human infectious diseases. In Infectious diseases: their evolution and eradication, ed. Cockburn, T.A., 84-107.

Springfield, IL: Charles Thomas, C. Cohen, M.L., and Tauxe, R.V. 1986. Drug-resistant Salmonella in the United States: An epidemiologic perspective. Science 234, 964-969.

Cole, D.J., Hill, V.R., Humenik, F.J., and Sobsey, M.D. 1999. Health, safety, and environmental concerns of farm animal waste. State Art. Rev. Occup. Med. 14, 423-448.

Cole, D., Todd, L., and Wing, S. 2000. Concentrated swine feeding operations and public health: A review of occupational and community health effects. Environ. Health Perspect. 10 8, 685-699.

Collinge, J. 1999. Variant Creutzfeldt-Jakob disease. Lancet 354, 317-323.

Collins, C.H. 2000. The bovine tubercle bacillus. Br. J. Biomed. Sci. 57, 234-240.

Coluzzi, M. 1999. The clay feet of the malaria giant and its African roots: Hypotheses and inferences about origin, spread and control of Plasmodium falciparum. Parassitologia 41, 277-283.

Cools, I., Uyttendaele, M., Cerpentier, J., D'Haese, E., Nelis, H.J., and Debevere, J. 2005. Persistence of Campylobacter jejuni on surfaces in a processing environment and on cutting boards. Lett. Appl. Microbiol. 40, 418-423.

Cooper, G.L., Venables, L.M., and Lever, M.S. 1996. Airborne challenge of chickens vaccinated orally with the genetically-defined Salmonella enteritidis aroA strain CVL30. Vet. Rec. 139, 447-448.

Cosivi, O., Grange, J.M., Daborn, C.J., Ravigilone, M.C., Fujikura, T., Cousins, D., Robinson, R.A., Huchzermeyer, H.F.A.K., De Kanntor, I., and Meslin, F.X. 1998. Zoonotic tuberculosis due to Mycobacterium bovis in developing countries. Emerg. Infect. Dis. 4, 59-70.

Coulanges, V., Andre, P., and Vidon, D.J. 1998. Effect of siderophores, catecholamines, and catechol compounds on Listeria spp. growth in iron-complexed medium. Biochem. Biophys. Res. Commun. 249, 526-530.

Courgnaud, V., Van Dooren, S., Liegeois, F., Pourrut, X., Abela, B., Loul, S., Mpoudi-Ngole, E., Vandamme, A., Delaporte, E., and Peeters, M. 2004. Simian T-cell leukemia virus (STLV) infection in wild primate populations in Cameroon: Evidence for dual STLV type 1 and type 3 infection in agile mangabeys (Cercocebus agilis). J. Virol. 78, 4700-4709.

Cox, N.J., and Subbarao, K. 2000. Global epidemiology of influenza: Past and present. Annu. Rev. Med. $51,407-421$.

Craig, J.V. 1978. Aggressive behavior of chickens: Some effects of social and physical environments. Department of Animal Sciences and Industry, Kansas State University. Presented at the 27th Annual National Breeder's Roundtable, May 11, Kansas City.

Crawford, P.C., Dubovi, E.J., Castleman,W.L., Stephenson, I., Gibbs, E.P., Chen, L., Smith, C., Hill, R.C., Ferro, P., Pompey, J., Bright, R.A., Medina, M.J., C.M. Johnson, Olsen, C.W., Cox, N.J., Klimov, A.I., Katz, J.M., and Donis, R.O. 2005. Transmission of equine influenza virus to dogs. Science 310, 482-485.

Crews, J. 2004. Handle with care: Transporting livestock correctly pays off. Food Systems Insider, March 1. <foodsystemsinsider.com/articles/0403/0403handle.htm>.

Cristalli, A., and Capua, I. 2007. Practical problems in controlling H5N1 high pathogenicity avian influenza at village level in Vietnam and introduction of biosecurity measures. Avian Dis. 51, 461-462.

Crump, J.A., Griffin, P.M., and Angulo, F.J. 2002. Bacterial contamination of animal feed and its relationship to human foodborne illness. Clin. Infect. Dis. 35, 859-865. 
Dargatz, D.A., Strohmeyer, R.A., Morley, P.S., Hyatt, D.R., and Salman, M.D. 2005. Characterization of Escherichia coli and Salmonella enterica from cattle feed ingredients. Foodborne Pathog. Dis. 2, 341-347.

Das, P. 2002. Interview: Michael Osterholm: Medical detective to fighting bioterrorism. Lancet Infect. Dis. 2, 502-505.

Daszak, P., and Cunningham, A.A. 2002. Emerging infectious diseases: A key role for conservation medicine. In Conservation medicine: Ecological health in practice, eds. Aguirre, A.A., Ostfeld, R.S., Tabor, G.M., House, C., and Pearl, M.C., 40-61. New York, NY: Oxford University Press.

Daszak, P., Cunningham, A.A., and Hyatt, A.D. 2000. Emerging infectious diseases of wildlife: Threats to biodiversity and human health. Science $287,443-449$.

Davis, M. 2005. The Monster at Our Door: The Global Threat of Avian Flu. New York, NY: The New Press.

Davis, R.J., and Lederberg, J., eds. 2001. Emerging Infectious Diseases from the Global to the Local Perspective: A Summary of a Workshop of the Forum of Emerging Infections. Washington, D.C.: National Academies Press.

Dawkins, M.S., and Hardie, S. 1989. Space needs of laying hens. Br. Poult. Sci. 30, 413-416.

de Groot, J., H.W. Moonen-Leusen, Thomas, G., Bianchi, A.T., Koolhaas, J.M., and van Milligen, F.J. 1999. Effects of mild stress on the immune response against pseudorabies virus in mice. Vet. Immunol. Immunopathol. 67, 153-160.

de Groot, J., Ruis, M.A., Scholten, J.W., Koolhaas, J.M., and Boersma, W.J. 2001. Long-term effects of social stress on antiviral immunity in pigs. Physiol. Behav. 73, 145-158.

De Groote, D., Ducatelle, R., and Haesebrouck, F. 2000. Helicobacters of possible zoonotic origin: A review. Acta. Gastroenterol. Belg. 63, 380-387.

De Haan, C., Steinfeld, H., and Blackburn, H. 1997. Livestock and the environment: Finding a balance. Brussels, Belgium: European Commission Directorate-General for Development. $<$ fao.org/docrep/x5303e/x5303e00.htm>.

de Jong, M.D., Simmons, C.P., Thanh, T.T., Hien, V.M., Smith, G.J., Chau, T.N., Hoang, D.M., Chau, N.V., Khanh, T.H., Dong, V.C., Qui, P.T., Cam, B.V., Q. Ha do, Y. Guan, Peiris, J.S., Chinh, N.T., Hien, T.T., and Farrar, J. 2006. Fatal outcome of human influenza A (H5N1) is associated with high viral load and hypercytokinemia. Nature Med. 12, 1203-1207.

de Jong, M.D., Van Cam, B., Qui, P.T., Hien, V.M., Thanh, T.T., Hue, N.B., M. Beld, Phuong, L.T., Khanh, T.H., Chau, N.V.V., Hien, T.T., Ha, D.Q., and Farrar, J. 2005. Fatal avian influenza A (H5N1) in a child presenting with diarrhea followed by coma. N. Engl. J. Med. 352, 686-691.

De Souza, R., Williams, J.S., and Meyerson, F.A.B. 2003. Critical links: Population, health and the environment. Popul. Bull. 58, 1-44.

Dean-Nystrom, E.A., Bosworth, B.T., Moon, H.W., and O'Brien, A.D. 1998. Bovine infection with Shigatoxin producing Escherichia coli. In Escherichia coli O157:H7 and other Shiga toxin-producing E. coli strains, eds. Kaper, J.B. and O'Brien, A.D., 261-267. Washington, D.C.: American Society for Microbiology.

Dee, S.A., Carlson, A.R., Winkelman, N.L., and Corey, M.M. 1993. Effect of management practices on the Streptococcus suis carrier rate in nursery pigs. J. Am. Vet. Med. Assoc. 20 3, 295-299.

DeFries, R.S., Houghton, R.A., Hansen, M.C., Field, C.B., Skole, D., and Townshend, J. 2002. Carbon emissions from tropical deforestation and regrowth based on satellite observations for the 1980s and 1990s. Proc. Natl. Acad. Sci. USA 99, 14256-14261.

Delgado, C.L., and Narrod, C.A. 2002. Impact of Changing Market Forces and Policies on Structural Change in the Livestock Industries of Selected Fast-Growing Developing Countries. Final Research Report of Phase I-Project on Livestock Industrialization, Trade, and Social-HealthEnvironment Impacts in Developing Countries. Rome: International Food Policy Research Institute (IFPRI) and Food and Agriculture Organization (FAO).

Delgado, C.L., and Narrod, C.A. 2003. Policy, technical, and environmental determinants and implications of the scaling-up of livestock production in four fast-growing developing countries: a synthesis. International Food Policy Research Institute.

Delgado, C., Rosegrant, M., Steinfeld, H., Ehui, S., and Courbois, C. 1999. Livestock to 2020, The next food revolution. Food, agriculture, and the environment discussion paper 28, for the International Food Policy Research Institute, Food and Agriculture Organization of the United Nations, and the International Livestock Research Institute. 
Department for Environment, Food and Rural Affairs (DEFRA). 2005. Epidemiology report published on H5N1 in Essex quarantine. DEFRA News Release, November 15.

Diamond, J. 1997. Guns, Germs and Steel: The Fates of Human Societies. New York, NY: Norton \& Company.

Diamond, J. 2002. Evolution, consequences and future of plant and animal domestication. Nature 418, 700-707.

Dierauf, L. 2004.Avian influenza in wild birds. U.S. Geological Survey National Wildlife Health Center. Wildlife Health Bulletin 04-01.

Dimmock, N.J., Easton, A., and Leppard, K. 2001. Introduction to Modern Virology, 5th ed. Malden, MA: Blackwell Publishing.

Dobson, A.P., and Carper, E.R. 1996. Infectious diseases and human population history. Bioscience Disease Ecolology 46, 115-126.

Domenech, J., Lubroth, J., Eddi, C., Martin,V., and Roger, F. 2006. Regional and international approaches on prevention and control of animal transboundary and emerging diseases. Ann. NY Acad. Sci. 10 81, 90-107.

Domingo, E., Baranowski, E., Ruiz-Jarabo, C.M., Martin-Hernandez, A.M., Saiz, J.C., and Escarmis, C. 1998. Quasispecies structure and persistence of RNA viruses. Emerg. Infect. Dis. 4, 521-527.

Domingo, E., Martinez-Salas, E., Sobrino, F., de la Torre, J.C., Portela, A., Ortin, J., C. Lopez-Galindez, P. Perez-Brena, Villanueva, N., Najera, R., et al. 1985. The quasispecies (extremely heterogeneous) nature of viral RNA genome populations-biological relevance: A review. Gene 40, 1-8.

Domingo, E., L. Menendez-Arias, and Holland, J.J. 1997. RNA virus fitness. Rev. Med. Virol. 7, 87-96.

Donnelly, C., Ghani, A., Leung, G., Hedley, A., Fraser, C., Riley, S., Abu-Raddad, L., Ho, L., Thach, T., and Chau, P. 2003. Epidemiological determinants of spread of causal agent of severe acute respiratory syndrome in Hong Kong. Lancet 361, 1761-1766.

Dore, M.P., Sepulveda, A.R., El-Zimaity, H., Yamaoka, Y., Osato, M.S., Mototsugu, K., Nieddu, A.M., Realdi, G., and Graham, D.Y. 2001. Isolation of Helicobacter pylori from sheep: Implications for transmission to humans. Am. J. Gastroenterol. 96, 1396-1401.

Doyle, M.P., and Erickson, M.C. 2006. Reducing the carriage of foodborne pathogens in livestock and poultry. Poult. Sci. 85, 960-973.

Drexler, M. 2002. Secret Agents: The Menace of Emerging Infections. Washington, D.C.: Joseph Henry Press.

Du, W. 2005. Streptococcus suis, (S. suis) pork production and safety. Ontario Ministry of Agriculture, Food and Rural Affairs.

Du Ry van Beest Holle, M., Meijer, A., Koopmans, M., and de Jager, C.M. 2005. Human-to-human transmission of avian influenza A/H7N7, The Netherlands, 2003. Euro. Surveill. 10, 264-268.

Dubos, R. 1965. Man Adapting. Binghamton, NY: The Vail-Ballou Press.

Duckworth, J.W., Salter, R.E., and Khounboline, K., eds. 1999. Wildlife in Lao PDR: 1999 Status Report.Vientiane: TheWorld Conservation Union (IUCN), Wildlife Conservation Society, and Centre for Protected Areas andWatershed Management.

Dunne, E.F., Fey, P.D., Kludt, P., Reporter, R., Mostashari, F., Shillam, P., Wicklund, J., Miller, C., Holland, B., Stamey, K., Barrett, T.J., Rasheed, J.K., Tenover, F.C., Ribot, E.M., and Angulo, F.J. 2000. Emergence of domestically acquired ceftriaxone-resistant Salmonella infections associated with ampC beta-lactamase. JAMA 284, 3151-3156.

Duplantier, J.M., Duchemin, J.B., Chanteau, S., and Carniel, E. 2005. From the recent lessons of the Malagasy foci towards a global understanding of the factors involved in plague reemergence. Vet. Res. 36, 437-453.

Ebert, D. 1995.Variation in parasite virulence is not an indicator for the evolution of benevolence. Conserv. Biol. 9, 1652-1653.

Ebert, D. 1998. Experimental evolution of parasites. Science 282, 1432-1435.

Ebert, D., and Hamilton, W.D. 1996. Sex against virulence: the coevolution of parasitic diseases. Trends Ecol. Evolut. 11, 79-82.

Ebert, D., and Mangin, K.L. 1997. The influence of host demography on the evolution of virulence of a microsporidian gut parasite. Evolution 51, 1828-1837. 
El-Lethey, H., Huber-Eicher, B., and Jungi, T.W. 2003. Exploration of stress-induced immunosuppression in chickens reveals both stress-resistant and stress-susceptible antigen responses. Vet. Immunol. Immunopathol. 95, 91-101.

Elfadil, A.A.,Vaillancourt, J.P., Meek, A.H., and Gyles, C.L. 1996.Aprospective study of cellulitis in broiler chickens in southern Ontario. Avian Dis. 40, 677-689.

Enøe, C., Mousing, J., Schirmer, A.L., and Willeberg, P. 2002. Infectious and rearing-system related risk factors for chronic pleuritis in slaughter pigs. Prev. Vet. Med. 54, 337-349.

Enria, D.A., and Feuillade, M.R. 1998. Argentine haemorrhagic fever (Juninvirus, Arenaviridae): A review on clinical, epidemiological, ecological, treatment and preventive aspects of the disease. In An overview of arbovirology in Brazil and neighboring countries, eds. Travassos da Rosa, A.P.A., Vasconcelos, P.F.C., and Travassos da Rosa, J.F.S., 219-232. Belem, Brazil: Instituto Evandro Chaggas.

Enserink, M. 2005. Veterinary scientists shore up defenses against bird flu. Science 30 8, 341.

Escudero, J.A., San Millan, A., Catalan, A., de la Campa, A.G., Rivero, E., Lopez, G., Dominguez, L., Moreno, M.A., and Gonzalez-Zorn, B., 2007. First characterization of fluoroquinolone resistance in Streptococcus suis. Antimicrob. Agents Chemother. 51, 777-782.

European Centre for Disease Control and Prevention (ECDC). 2007. Low pathogenicity avian influenza (LPAI): Human cases recorded in the literature.

European Commission Health and Consumer Protection Directorate General (DG-SANCO). 2000. The welfare of animals during transport, 14-19, adopted March 11.

European Commission Health and Consumer Protection Directorate General (DG-SANCO). 2002. General scientific steering committee opinion on the implications of the recent papers on transmission of BSE by blood. European Commission, September 13.

European Commission Scientific Veterinary Committee (SVC). 1996. Report on the Welfare of Laying Hens. Brussels, Belgium: Scientific Veterinary Committee.

Evans, A.S. 1955. Pathogenicity and immunology of Newcastle disease virus (NVD) in man. Am. J. Public Health Nations Health 45, 742-745.

Ewald, C., Heer, A., and Havenith, U. 1994. Factors associated with the occurrence of influenza A virus infections in fattening swine. Berl. Munch. Tierarztl. Wochenschr. 10 7, 256-262.

Ewald, P.W. 1994. Evolution of Infectious Disease. New York, NY: Oxford University Press.

Ewald, P.W. 1995. The evolution of virulence: A unifying link between parasitology and ecology. J. Parasitol. 81, 659-669.

Ewald, P.W. 1996. Guarding against the most dangerous emerging pathogens. Emerg. Infect. Dis. 2, 245-257.

Ewers, C., Li, G., Wilking, H., Kiessling, S., Alt, K., Antao, E.M., Laturnus, C., Diehl, I., Glodde, S., Homeier, T., Bohnke, U., Steinruck, H., Philipp, H.C., andWieler, L.H. 2007. Avian pathogenic, uropathogenic, and newborn meningitis-causing Escherichia coli: Howclosely related are they? Int. J. Med. Microbiol. 297, 163-176.

Fa, J.E., and Garc'ia-Yuste, J.E. 2001. Commercial bushmeat hunting in the Monte Mitra forests, Equatorial Guinea: Extent and impact. Anim. Biodiv. Conserv. 24, 31-52.

Fa, J.E., Peres, C.A., and Meeuwig, J. 2002. Bushmeat exploitation in tropical forests: An intercontinental comparison. Conserv. Biol. 16, 232-237.

Fa, J.E., Juste, J., Delval, J.P., and Castroviejo, J. 1995. Impact of market hunting on mammal species in Equatorial-Guinea. Conserv. Biol. 9, 1107-1115.

Fairchild, B.D. 2005. Broiler production systems: The ideal stocking density? The University of Georgia, College of Agricultural and Environmental Sciences, Department of Poultry Science.

Fan, E.Q. 2003. SARS: Economic Impacts and Implications. Asian Development Bank. Hong Kong: Oxford University Press for the Asian Development Bank.

Favoretto, S.R., de Mattos, C.C., Morais, N.B., Alves Araujo, F.A., and de Mattos, C.A., 2001. Rabies in marmosets (Callithrix jacchus), Cear'a, Brazil. Emerg. Infect. Dis. 7, 1062-1065.

Feagins, A.R., Opriessnig, T., Guenette, D.K., Halbur, P.G., and Meng, X.J. 2007. Detection and characterization of infectious Hepatitis $E$ virus from commercial pig livers sold in local grocery stores in the USA. J. Gen. Virol. 88, 912-917.

Feldgarden, M. 2007. Forum-Cefquinome: When regulation collides with biology. Microbe Magazine, June. 
Feldmann, A., Schafer, M.K., Garten,W., and Klenk, H.D. 2000. Targeted infection of endothelial cells by avian influenza virus A/FPV/Rostock/34 (H7N1) in chicken embryos. J. Virol. 74, 8018-8027.

Fenlon, D.R. 1999. Listeria monocytogenes in the natural environment. In Listeria, listeriosis, and food safety, 2nd ed., eds. Ryser, E.T. and Marth, E.H., 21-38. New York, NY: Marcel Dekker Inc.

Fey, P.D., Safranek, T.J., Rupp, M.E., Dunne, E.F., Ribot, E., Iwen, P.C., P.A. Bradford, Angulo, F.J., and Hinrichs, S.H. 2000. Ceftriaxone-resistant Salmonella infection acquired by a child from cattle. N. Engl. J. Med. 342, 1242-1249.

Field, H., Young, P., Yob, J.M., Mills, J., Hall, L., and Mackenzie, J. 2001. The natural history of Hendra and Nipah viruses. Microb. Infect. 3, 307-314.

Fitchner, G.J. 1987. The Pennsylvania/Virginia experience in eradication of avian influenza H5N2. Proceedings of the 2nd International Symposium on Avian Influenza, 33-38. Athens, GA: U.S. Animal Health Association.

Fleming, D.W., Cochi, M.D., MacDonald, K.L., Brondum, J., Hayes, P.S., Plikaytis, B.D., Holmes, M.B., Audurier, A., Broome, C.V., and Reingold, A.L. 1985. Pasteurized milk as a vehicle of infection in an outbreak of listeriosis. N. Engl. J. Med. 312, 404-407.

Fontenot, J.P. 2001. Utilization of poultry litter as feed for beef cattle. Food and Drug Administration Public Hearing on Animal Feeding Regulation, October 30.

Fontenot, J.P.,Webb, K.E., Jr., Harmon, B.W., Tucker, R.E., and Moore, W.E.C. 1971. Studies of processing, nutritional value and palatability of broiler litter for ruminants. In Proceedings of the International Symposium on Livestock Wastes, 271-301. St. Joseph, Michigan: American Society of Agricultural Engineers.

Food and Agriculture Organization of the United Nations (FAO). 1998. Industrial livestock production, concentrate feed demand and natural resource requirements in China Ke Bingsheng. Beijing, China: China Agricultural University.

Food and Agriculture Organization of the United Nations (FAO). 1999. Poverty alleviation and food security in Asia: Role of livestock. Bangkok, Thailand: FAO Regional Office for Asia and the Pacific. Food and Agriculture Organization of the United Nations (FAO). 2000. The State of World Fisheries and Aquaculture. Rome, Italy: Food and Agriculture Organization of the United Nations.

Food and Agriculture Organization of the United Nations (FAO). 2002. Improved animal health for poverty reduction and sustainable livelihoods. FAO animal production and health paper 153.

Food and Agriculture Organization of the United Nations (FAO). 2004a. Loss of domestic animal breeds alarming. Press release, March 31.

Food and Agriculture Organization of the United Nations (FAO). 2004b. Update on the avian influenza situation 5, February 25.

Food and Agriculture Organization of the United Nations (FAO). 2006a. Production of meat and share in world. In FAO Statistical Yearbook. Rome, Italy: Food and Agriculture Organization.

Food and Agriculture Organization of the United Nations (FAO). 2006b. Should wild birds now be considered a permanent reservoir of the virus? FAO Avian Influenza Disease Emergency News 40, 1-11.

Food and Agriculture Organization of the United Nations (FAO). 2007a. Avian influenza-questions \& answers: The facts of bird flu. Agriculture Department, Animal Production and Health Division.

Food and Agriculture Organization of the United Nations (FAO). 2007b. FAO-STAT. Rome, Italy.

Formenty, P., Boesch, C., Wyers, M., Steiner, C., Donati, F., Dind, F., Walker, F., and Le Guenno, B. 1999. Ebola virus outbreak among wild chimpanzees living in a rain forest of Cote d'Ivoire. J. Infect. Dis. 179, S120-S126.

Fouchier, R.A.M., Munster, V., Wallensten, A., Bestebroer, T.M., Herfst, S., Smith, D., Rimmelzwaan, G.F., Olsen, B., and Osterhaus, A.D.M.E. 2005. Characterization of a novel influenza A virus hemagglutinin subtype (H16) obtained from black-headed gulls. J. Virol. 79, 2814-2822.

Fouchier, R.A., Schneeberger, P.M., Rozendaal, F.W., Broekman, J.M., Kemink, S.A., Munster, V., Kuiken, T., Rimmelzwaan, G.F., Schutten, M.,Van Doornum, G.J., Koch, G., Bosman, A., Koopmans, M., and Osterhaus, A.D. 2004. Avian influenza A virus (H7N7) associated with human conjunctivitis and a fatal case of acute respiratory distress syndrome. Proc. Natl. Acad. Sci. USA $101,1356-1361$.

Foxman, B. 2002. Epidemiology of urinary tract infections: Incidence, morbidity, and economic costs. Am. J. Med. 113, 5S-13S. 
Frank, S.A., and Jeffrey, J.S. 2001. The probability of severe disease in zoonotic and commensal infections. Proc. Biol. Sci. 268, 53-60.

Fraser, D.W., Tsai, T.R., Orenstein, W., Parkin, W.E., Beecham, H.J., Sharrar, R.G., Harris, J., Mallison, G.F., Martin, S.M., McDade, J.E., Shepard, C.C., and Brachman, P.S. 1977. Legionnaires' disease: Description of an epidemic of pneumonia. N. Engl. J. Med. 297, 1189-1197.

Freeman, B.M., Manning, A.C.C., Harrison, G.F., and Coates, M.E. 1975. Dietary aureomycin and the response of the fowl to stressors. Br. Poult. Sci. 16, 395-404.

Fricker, J. 2000. Emerging infectious diseases: A global problem. Mol. Med. Today 6, 334-335.

Friedman, C.R., Neimann, J., Waegener, H.G., and Tauxe, R.V. 2000. Epidemiology of Campylobacter jejuni infections in the United States and other industrialized nations. In Campylobacter, 2nd edition, eds. Nachamkin, I. and Blaser, M.J., 121-139. Washington, D.C.: American Society for Microbiology.

Friend, M., McLean, R.J., and Dein, F.J. 2001. Disease emergence in birds: Challenges for the twentyfirst century. The Auk. 118, 290-303.

Frost,W.H. 1920. Statistics of influenza morbidity. Public Health Rep. 35, 584-597.

Fujimoto, S., Kobayashi, M., Uemura, O., Iwasa, M., Ando, T., Katoh, T., Nakamura, C., Maki, N., Togari, H., and Wada, Y. 1998. PCR on cerebrospinal fluid to show influenza-associated acute encephalopathy or encephalitis. Lancet 352, 873-875.

Furushita, M., Shiba, T., Maeda, T., Yahata, M., Kaneoka, A., Takahashi, Y., Torii, K., Hasegawa, T., and Ohta, M. 2003. Similarity of tetracycline resistance genes isolated from fish farm bacteria to those from clinical isolates. Appl. Environ. Microbiol. 69, 5336-5342.

Fussell, L. 1998. Poultry industry strategies for control of immunosuppressive diseases. Poult. Sci. 77, 1193-1196.

Gabriel, G., Dauber, B., Wolff, T., Planz, O., Klenk, H.D., and Stech, J. 2005. The viral polymerase mediates adaptation of an avian influenza virus to a mammalian host. Proc. Natl. Acad. Sci. USA $102,18590-18595$.

Galimand, M., Guiyoule, A., Gerbaud, G., Rasoamanana, B., Chanteau, S., Carniel, E., and Courvalin, P. 1997. Multidrug resistance in Yersinia pestis mediated by a transferable plasmid. N. Engl. J. Med. 337, 677-680.

Gambaryan, A.S., Tuzikov, A.B., Bovin, N.V., Yamnikova, S.S., Lvov, D.K., Webster, R.G., and Matrosovich, M.N. 2003. Differences between influenza virus receptors on target cells of duck and chicken and receptor specificity of the 1997 H5N1 chicken and human influenza viruses from Hong Kong. Avian Dis. 47, 1154-1160.

Gambaryan, A., Tuzikov, A., Pazynina, G., Bovin, N., Balish, A., and Klimov, A. 2006. Evolution of the receptor binding phenotype of influenza A (H5) viruses. Virology 344, 432-438.

Gambaryan, A.S., Tuzikov, A.B., Pazynina, G.V., Webster, R.G., Matrosovich, M.N., and Bovin, N.V. 2004. H5N1 chicken influenza viruses display a high binding affinity for Neu5Acalpha23Galbeta1-4(6-HSO3)GlcNAc-containing receptors. Virology 326, 310-316.

Gambaryan, A., Webster, R., and Matrosovich, M. 2002. Differences between influenza virus receptors on target cells of duck and chicken. Arch. Virol. 147, 1197-1208.

Gambaryan, A., Yamnikova, S., Lvov, D., Tuzikov, A., Chinarev, A., Pazynina, G., Webster, R., Matrosovich, M., and Bovin, N. 2005. Receptor specificity of influenza viruses from birds and mammals: New data on involvement of the inner fragments of the carbohydrate chain. Virology 334, 276-283.

Garcia, M., Crawford, J.M., Latimer, J.W., Rivera-Cruz, M.V.Z.E., and Perdue, M.L. 1996. Heterogeneity in the hemagglutinin gene and emergence of the highly pathogenic phenotype among recent H5N2 avian influenza viruses from Mexico. J. Gen. Virol. 77, 1493-1504.

Garofalo, C., Vignaroli, C., Zandri, G., Aquilanti, L., Bordoni, D., Osimani, A., Clementi, F., and Biavasco, F. 2007. Direct detection of antibiotic resistance genes in specimens of chicken and pork meat. Int. J. Food Microbiol. 113, 75-83.

Garrett, L. 1994. The Coming Plague: Newly Emerging Diseases in a World Out of Balance. New York, NY: Farrar, Straus, Giroux.

Gast, R.K., and Beard, C.W. 1990. Production of Salmonella enteritidis-contaminated eggs by experimentally infected hens. Avian Dis. 34, 438-446.

Gauthier-Clerc, M., Lebarbenchon, C., and Thomas, F. 2007. Recent expansion of highly pathogenic avian influenza H5N1: A critical review. Ibis 149, 202-214. 
Gibbs Jr., C.J., Gajdusek, D.C., and Latarjet, R. 1978. Unusual resistance to ionizing radiation of the viruses of kuru, Creutzfeldt-Jakob disease, and scrapie. Proc. Natl. Acad. Sci. USA. 75, 62686270 .

Gilbert, M., Chaitaweesub, P., Parakamawongsa, T., Premashthira, S., Tiensin, T., Kalpravidh,W.,Wagner, H., and Slingenbergh, J. 2006. Free-grazing ducks and highly pathogenic avian influenza, Thailand. Emerg. Infect. Dis. 12, 227-234.

Gilbert, M., Wint, W., and Slingenbergh, J. 2004. The ecology of highly pathogenic avian influenza in East and Southeast Asia: Outbreaks distribution, risk factors and policy implications. Consultancy report for the Animal Health Service of the Animal Production and Health Division of the Food and Agriculture Organization of the United Nations.

Gill, D.E., and Mock, B.A. 1985. The ecological and evolutionary dynamics of parasites: The case of Trypanosoma diemyctyli in the red-spotted newt, Notophthalmus viridescens. In Ecology and genetics of host-parasite interactions (Linnean Society Symposium Series, Number 11), eds. Rollinson, D. and Anderson, R.M., 157-184. London, UK: Academic Press.

Gill, J.S., Webby, R., Gilchrist, M.J., and Gray, G.C. 2006. Avian influenza among waterfowl hunters and wildlife professionals. Emerg. Infect. Dis. 12, 1284-1286.

Glaser, L., Stevens, J., Zamarin, D., Wilson, I.A., Garcia-Sastre, A., T.M. Tumpey, Basler, C.F., Taubenberger, J.K., and Palese, P. 2005. A single amino acid substitution in 1918 influenza virus hemagglutinin changes receptor binding specificity. J. Virol. 79, 11533-11536.

Glass, G.E., Yates, T.L., Fine, J.B., Shields, T.M., Kendall, J.B., Hope, A.G., Parmenter, C.A., Peters, C.J., Ksiazek, T.G., Li, C.S., Patz, J.A., and Mills, J.N. 2002. Satellite imagery characterizes local animal reservoir populations of Sin Nombre virus in the southwestern United States. Proc. Natl. Acad. Sci. USA 99, 16817-16822.

Godfroid, J., Cloeckaert, A., Liautard, J.P., Kohler, S., Fretin, D.,Walravens, K., B. Garin-Bastuji, and Letesson, J.-J. 2005. From the discovery of the Malta fever's agent to the discovery of a marine mammal reservoir, brucellosis has continuously been a re-emerging zoonosis. Vet. Res. 36, 313326.

Goh, S.H., Driedger, D., Gillett, S., Low, D.E., Hemmingsen, S.M., Amos, M., Chan, D., Lovgren, M., Willey, B.M., Shaw, C., and Smith, J.A. 1998. Streptococcus iniae, a human and animal pathogen: Specific identification by the chaperonin 60 gene identification method. J. Clin. Microbiol. 36, 2164-2166.

Goto, H., and Kawaoka, Y. 1998. A novel mechanism for the acquisition of virulence by a human influenza A virus. Proc. Natl. Acad. Sci. USA 95, 10224-10228.

Gottschalk, M. 2004. Porcine Streptococcus suis strains as potential sources of infections in humans: An underdiagnosed problem in North America? J. Swine Health Product. 12, 197-199.

Gottschalk, M., and Segura, M. 2000. The pathogenesis of the meningitis caused by Streptococcus suis: The unresolved questions. Vet. Microbiol. 76, 259-272.

Gould, A.R. 1996. Comparison of the deduced matrix and fusion protein sequences of equine morbillivirus with cognate genes of the Paramyxoviridae. Virus Res. 43, 17-31.

Govorkova, E.A., Rehg, J.E., Krauss, S.,Yen, H.L., Guan,Y., Peiris, M., Nguyen, T.D., Hanh, T.H., Puthavathana, P., Long, H.T., Buranathai, C., Lim, W., Webster, R.G., and Hoffmann, E. 2005. Lethality to ferrets of H5N1 influenza viruses isolated from humans and poultry in 2004. J. Virol. 79, 2191-2198. Erratum in J. Virol. 80, 6195.

Graham, J.P., Boland, J.J., and Silbergeld, E. 2007. Growth promoting antibiotics in food animal production: An economic analysis. Public Health Rep. 122, 79-87.

Graves, L.M., Hunter, S.B., Ong, A.R., Schoonmaker-Bopp, D., Hise, K., Kornstein, L., DeWitt, W.E., Hayes, P.S., Dunne, E., Mead, P., and Swaminathan, B. 2005. Microbiological aspects of the investigation that traced the 1998 outbreak of listeriosis in the United States to contaminated hot dogs and establishment of molecular subtyping-based surveillance for Listeria monocytogenes in the PulseNet network. J. Clin. Microbiol. 43, 2350-2355.

Gray, M.J., Freitag, N.E., and Boor, K.J. 2006. How the bacterial pathogen Listeria monocytogenes mediates the switch from environmental Dr. Jekyll to pathogenic Mr. Hyde. Infect. Immun. 74, 2505-2512.

Greger, M. 2006. Bird Flu: A Virus of Our Own Hatching. New York, NY: Lantern Books, xii. 
Gregory, E., Barnhart, H., Dreesen, D.W., Stern, N.J., and Corn, J.L. 1997. Epidemiological study of Campylobacter spp. in Broilers: Source, time of colonization, and prevalence. Avian Dis. Avian Dis. 41, 890-898.

Greiner, M., Muller-Graf, C., Hiller, P., Schrader, C., Gervelmeyer, A., L. Ellerbroek, and Appel, B. 2007. Expert opinion based modelling of the risk of human infection with $\mathrm{H} 5 \mathrm{~N} 1$ through the consumption of poultry meat in Germany. Berl. Munch. Tierarztl. Wochenschr. 120, 98-107.

Gross, E. 2003. Update: Multistate outbreak of monkeypox-Illinois, Indiana, Kansas, Missouri, Ohio, and Wisconsin. Ann. Emerg. Med. 42, 660-664.

Gross,W.B., and Siegel, P.B. 1988. Environment-genetic influences on immunocompetence. J. Anim. Sci. 66, 2091-2094.

Guan,Y., Peiris, J.S., Lipatov, A.S., Ellis,T.M., Dyrting, K.C., Krauss, S., Zhang, L.J., Webster, R.G., and Shortridge, K.F. 2002. Emergence of multiple genotypes of H5N1 avian influenza viruses in Hong Kong SAR. Proc. Natl. Acad. Sci. USA 99, 8950-8955.

Guan, Y., Zheng, B.J., He, Y.Q., Liu, X.L., Zhuang, Z.X., Cheung, C.L., S.W. Luo, Li, P.H., Zhang, L.J., Guan, Y.J., Butt, K.M., Wong, K.L., Chan, K.W., Lim, W., K.F. Shortridge, Yuen, K.Y., Peiris, J.S., and Poon, L.L. 2003. Isolation and characterization of viruses related to the SARS coronavirus from animals in southern China. Science $302,276-278$.

Gubareva, L.V., McCullers, J.A., Bethell, R.C., andWebster, R.G. 1998. Characterization of influenza A/HongKong/156/97 (H5N1) virus in a mouse model and protective effect of zanamivir on H5N1 infection in mice. J. Infect. Dis. 178, 1592-1596.

Gubser, C., Hue, S., Kellam, P., and Smith, G.L. 2004. Poxvirus genomes: A phylogenetic analysis. J. Gen. Virol. 85, 105-117.

Guenno, B.L. 1997. Haemorrhagic fevers and ecological perturbations. Arch. Virol. 13, A191-A199.

Gunter, C., and Dhand, R. 2005. The chimpanzee genome. Nature 437, 47.

Guo, Y.J., Krauss, S., Senne, D.A., Mo, I.P., Lo, K.S., Xiong, X.P., Norwood, M., Shortridge, K.F., Webster, R.G., and Guan, Y. 2000. Characterization of the pathogenicity of members of the newly established H9N2 influenza virus lineages in Asia. Virology 267, 279-288.

Guo, Y., Li, J., Cheng, X.,Wang, M., Zhou, Y., Li, C., Chai, F., Liao, H., Zhang, Y., Guo, J., Huang, L., and Bei, D. 1999. Discovery of men infected by avian influenza A (H9N2) virus. Chinese J. Exp. Clin. Virol. 13, 105-108.

Guo, C.T., Takahashi, N., Yagi, H., Kato, K., Takahashi, T., Yi, S.Q., Chen, Y., Ito, T., Otsuki, K., Kida, H., Kawaoka, Y., Hidari, K.I., Miyamoto, D., Suzuki, T., and Suzuki, Y. 2007. The quail and chicken intestine have sialyl-galactose sugar chains responsible for the binding of influenza $A$ viruses to human type receptors. Glycobiology 17, 713-724.

Gupta, A., Nelson, J.M., Barrett, T.J., Tauxe, R.V., Rossiter, S.P., Friedman, C.R., Joyce, K.W., Smith, K.E., Jones, T.F., Hawkins, M.A., Shiferaw, B., Beebe, J.L., Vugia, D.J., Rabatsky-Ehr, T., Benson, J.A., Root, T.P., and Angulo, F.J. 2004. Antimicrobial resistance among Campylobacter strains, United States, 1997-2001. Emerg. Infect. Dis. 10, 1102-1109.

Guthrie, A.J., Stevens, K.B., and Bosman, P.P. 1999. The circumstances surrounding the outbreak and spread of equine influenza in South Africa. Rev. Sci. Tech. Off. Int. Epiz. 18, 179-185.

Gylfe, A., Bergstrom, S., Lundstrom, J., and Olsen, B. 2000. Reactivation of Borrelia infection in birds. Nature 40 3, 724-725.

Gylys, L., Chomel, B.B., and Gardner, I.A. 1998. Epidemiological surveillance of rabies in Lithuania from 1986 to 1996. Rev. Sci. Tech. Off. Int. Epiz. 17, 691-698.

Haapapuro, E.R., Barnard, N.D., and Simon, M. 1997. Review: Animal waste used as livestock feed: Dangers to human health. Prev. Med. 26, 599-602.

Hafez, H.M. 2000. Factors influencing turkey diseases. World Poultry Turkey Health Special, 6-8.

Hahn, BH, Shaw, G.M., De Cock, K.M., and Sharp, P.M. 2000. AIDS as a zoonosis: Scientific and public health implications. Science 287, 607-614.

Haines, A., and Patz, J.A. 2004. Health effects of climate change. JAMA 291, 99-103.

Hall, C. 2004. Impact of avian influenza on U.S. poultry trade relations-2002: H5 or H7 low pathogenic avian influenza. Ann. NY Acad. Sci. 10 26, 47-53.

Halvorson, D.A., Kelleher, C.J., and Senne, D.A. 1985. Epizootiology of avian influenza: Effect of seasonal incidence in sentinel ducks and domestic turkey in Minnesota. Appl. Environ. Microbiol. 49, 914-919. 
Harbottle, H., White, D.G., McDermott, P.F., Walker, R.D., and Zhao, S. 2006. Comparison of multilocus sequence typing, pulsed-field gel electrophoresis, and antimicrobial susceptibility typing for characterization of Salmonella enterica serotype Newport isolates. J. Clin. Microbiol. 44, 24492457.

Harrison, P.J., and Roberts, G.W. 1992. How now mad cow. Br. Med. J. 304: 929-930.

Hatta, M., Gao, P., Halfmann, P., and Kawaoka,Y. 2001. Molecular basis for high virulence of Hong Kong H5N1 influenza A viruses. Science 293, 1840-1842.

Haus-Cheymol, R., Espie, E., Che, D., Vaillant, V., de Valk, H., and Desenclos, J.C. 2005. Association between indicators of cattle density and incidence of paediatric haemolytic-uraemic syndrome (HUS) in children under 15 years of age in France between 1996 and 2001: An ecological study. Epidemiol. Infect. 134, 1-7.

Hawken, R.J., Beattie, C.W., and Schook, L.B. 1998. Resolving the genetics of resistance to infectious diseases. Rev. Sci. Tech. Off. Int. Epiz. 17, 17-25.

Health Protection Agency. 2007. Confirmation of avian influenza H7N2 infection. May 25.

Heinitz, M.L., Ruble, R.D., Wagner, D.E., and Tatini, S.R. 2000. Incidence of Salmonella in fish and seafood. J. Food Prot. 63, 579-592.

Helms, M., Simonsen, J., Olsen, K.E., and Molbak, K. 2005. Adverse health events associated with antimicrobial drug resistance in Campylobacter species: A registry-based cohort study. J. Infect. Dis. 191, 1051.

Heneine, W., Switzer, W.M., Sandstrom, P., Brown, J., Vedapuri, S., Schable, C.A., Khan, A.S., Lerche, N.W., Schweizer, M., D. Neumann-Haefelin, Chapman, L.E., and Folks,T.M. 1998. Identification of a human population infected with simian foamy viruses. Nature Med. 4, 403-407.

Hennessy, T.W., Hedberg, C.W., Slutsker, L., White, K.E., Besser-Wiek, J.M., Moen, M.E., Feldman, J., Coleman, W.W., Edmonson, L.M., MacDonald, K.L., and Osterholm, M.T. 1996. A national outbreak of Salmonella enteritidis infections from ice cream. N. Engl. J. Med. 334, 1281-1286.

Heyder, J. 2004. Deposition of inhaled particles in the human respiratory tract and consequences for regional targeting in respiratory drug delivery. Proc. Am. Thorac. Soc. 1, 315-320.

Hilton, D.A., Ghani, A.C., Conyers, L., Edwards, P., McCardle, L., Penney, M., Ritchie, D., Ironside, J.W. 2002. Accumulation of prion protein in tonsil and appendix: review of tissue samples. BMJ 325 , 633-634.

Himathongkham, S., and Riemann, H. 1999. Destruction of Salmonella typhimurium, Escherichia coli 0157:H7 and Listeria monocytogenes in chicken manure by drying and/or gassing with ammonia. FEMS Microbiol. Lett. 171, 179-182.

Hinshaw, V.S., Bean, W.J., Webster, R.G., and Sriram, G. 1980a. Genetic reassortment of influenza A viruses in the intestinal tract of ducks. Virology $102,412-419$.

Hinshaw, V.S., Webster, R.G., Naeve, C.W., and Murphy, B.R. 1983. Altered tissue tropism of humanavian reassortant influenza viruses. Virology 128, 260-263.

Hinshaw, V.S., Webster, R.G., and Turner, B. 1980b. The perpetuation of orthomyxoviruses and paramyxoviruses in Canadian waterfowl. Can. J. Microbiol. 26, 622-629.

Hinshaw, V.S., Webster, R.G., and Turner, B. 1979. Water-borne transmission of influenza A viruses? Intervirology 11, 6668.

Hoberg, E.P., Alkire, N.L., de Queiroz, A., and Jones, A. 2001. Out of Africa: Origins of the Taenia tapeworms in humans. Proc. Biol. Sci. 268, 781-787.

Hofacre, C.L., White, D.G., Maurer, J.J., Morales, C., Lobsinger, C., and Hudson, C. 2001. Characterization of antibiotic-resistant bacteria in rendered animal products. Avian Dis. Avian Dis. 45, 953-961.

Hoff, B., and Smith III, C. 2000. Mapping Epidemics: A Historical Atlas of Disease. New York, NY: Grolier Publishing.

Hofmann, H., Geier, M., Marzi, A., Krumbiegel, M., Peipp, M., Fey, G.H., Gramberg, T., and Pohlmann, S. 2004. Susceptibility to SARS coronavirus $S$ protein-driven infection correlates with expression of angiotensin converting enzyme 2 and infection can be blocked by soluble receptor. Biochem. Biophys. Res. Commun. 319, 1216-1221.

Hofstaad, M.S., Calnek, B.W., Helmboldt, C.F., Reid, W.M., and Yoder, H.W., Jr. 1972. Diseases of Poultry. Ames, IA: lowa State University Press.

Holland, J.J., de la Torre, J.C., Clarke, D.K., and Duarte, E. 1991. Quantitation of relative fitness and great adaptability of clonal populations of RNA viruses. J. Virol. 65, 2960-2967. 
Hollenbeck, J.E. 2005. An avian connection as a catalyst to the 1918- 1919 influenza pandemic. Int. J. Med. Sci. 2, 87-90. hpubmedcentral. nih.gov/articlerender.fcgi?artid=1145139i.

Holmberg, S.D.,Wells, J.G., and Cohen, M.L. 1984. Animal-to-man transmission of antimicrobialresistant Salmonella: Investigations of outbreaks, U.S., 1971-1983. Science 225, 833-835.

Holmes, G.P., Chapman, L.E., Stewart, J.A., Straus, S.E., Hilliard, J.K., and Davenport, D.S. 1995. Guidelines for the prevention and treatment of B-virus infections in exposed persons. Clin. Infect. Dis. 20, 421-439.

Holmes, K.V. 1999. Coronaviruses. In Encyclopedia of virology, eds. Granoff, A., Webster, R.G., 291298. San Diego, CA: Academic Press.

Holt, P.S., Buhr, R.J., Cunningham, D.L., and Porter, R.E. 1994. Effect of two different molting procedures on a Salmonella enteritidis infection. Poult. Sci. 73, 1267-1275.

Holt, P.S., Mitchell,B.W., and Gast, R.K. 1998. Airborne horizontal transmission of Salmonella enteritidis in molted laying chickens. Avian Dis. 42, 45-52.

Hopp, P., H. Wahlstr"om, and Hirn, J. 1999. A common Salmonella Control Programme in Finland, Norway and Sweden. Acta Vet. Scand. Suppl. 91, 45-49.

Horimoto, T., and Kawaoka, Y. 2001. Pandemic threat posed by avian influenza A viruses. Clin. Microbiol. Rev. 14, 129-149.

Horimoto, T., Nakayama, K., Smeekens, S.P., and Kawaoka, Y. 1994. Proprotein-processing endoproteases PC6 and furin both activate hemagglutinin of virulent avian influenza viruses. $\mathrm{J}$. Virol. 68, 6074-6078.

Horimoto, T., Rivera, E., Pearson, J., Senne, D., Krauss, S., Kawaoka, Y., and Webster, R.G. 1995. Origin and molecular changes associated with emergence of a highly pathogenic H5N2 influenza virus in Mexico. Virology 213, 223-230.

Houston, F., Foster, J.D., Chong, A., Hunter, N., and Bostock, C.J. 2000. Transmission of BSE by blood transfusion in sheep. Lancet 356, 999-1000.

Hrudey, S.E., Payment, P., Huck, P.M., Gillham, R.W., and Hrudey, E.J. 2003. A fatal waterborne disease epidemic inWalkerton, Ontario: Comparison with other waterborne outbreaks in the developed world. Wat. Sci. Tech. 47, 7-14.

Hu, W.S., and Temin, H.M. 1990. Retroviral recombination and reverse transcription. Science 250, 12271233.

Huang, Y.T., Teng, L.J., Ho, S.W., and Hsueh, P.R. 2005. Streptococcus suis infection. J. Microbiol. Immunol. Infect. 38, 306-313.

Hugh-Jones, M.E., Hubbert,W.T., and Hagsad, H.V. 1995. Zoonoses: Recognition, Control, and Prevention. Ames, IA: lowa State University Press.

Hulse-Post, D.J., Sturm-Ramirez, K.M., Humberd, J., Seiler, P., Govorkova, E.A., Krauss, S., Scholtissek, C., Puthavathana, P., Buranathai, C., Nguyen, T.D., Long, H.T., Naipospos, T.S., Chen, H., Ellis, T.M., Guan, Y., Peiris, J.S., and Webster, R.G. 2005. Role of domestic ducks in the propagation and biological evolution of highly pathogenic H5N1 influenza viruses in Asia. Proc. Natl. Acad. Sci. USA 10 2, 10682-10687.

Humphrey, T. 2006. Are happy chickens safer chickens? Poultry welfare and disease susceptibility. $\mathrm{Br}$. Poult. Sci. 47, 379-391.

Humphrey, T.J. 1991. Food poisoning: A change in patterns? Vet. Annual 31, 32-37.

Humphrey, T.J., Greenwood, M., Gilbert, R.J., Rowe, B., and Chapman, P.A. 1989. The survival of salmonellas in shell eggs cooked under simulated domestic conditions. Epidemiol. Infect. 103 , 35-45.

Humphrey, T.J., Jorgensen, F., Frost, J.A., Wadda, H., Domingue, G., Elviss, N.C., Griggs, D.J., and Piddock, L.J. 2005. Prevalence and subtypes of ciprofloxacin-resistant Campylobacter spp. in commercial poultry flocks before, during, and after treatment with fluoroquinolones. Antimicrob. Agents Chemother. 49, 690-698.

Iglesias, G., and Trujano, M. 1992. Experimental replication of clinical disease associated with Streptococcus suis type 2. Agri-Practice 13, 25-30.

Iglesias, J.G., Trujano, M., and Xu, J. 1992. Inoculation of pigs with Streptococcus suis type 2 alone or in combination with pseudorabies virus. Am. J. Vet. Res. 53, 364-367.

Inglesby, T.V., Dennis, D.T., Henderson, D.A., Bartlett, J.G., Ascher, M.S., Eitzen, E., Fine, A.D., Friedlander, A.M., Hauer, J., Koerner, J.F., Layton, M., McDade, J., Osterholm, M.T., O'Toole, T., Parker, G., Perl, T.M., Russell, P.K., Schoch-Spana, M., and Tonat, K., for the Working Group on 
Civilian Biodefense. 2000. Plague as a biological weapon: Medical and public health management. JAMA 283, 2281-2290.

Institute of Medicine (IOM). 1988. Ensuring Safe Food: From Production to Consumption. Washington, D.C.: National Academies Press.

International Food Safety Authorities Network (IFSAN). 2004. Highly pathogenic avian influenza H5N1 outbreaks in poultry and humans: Food safety implications. INFOSAN information note no. 2/04avian influenza.

lowa Egg Council. 2002. Production. hiowaegg.org/allabouteggs/production.htmli.

Ip, M., Fung, K.S.C., Chi, F., Cheuk, E.S.C., Chau, S.S.L., Wong, B.W.H., Lui, S., Hui, M., Lai, R.W.M., and Chan, P.K.S. 2007. Streptococcus suis in Hong Kong. Diagn. Microbiol. Infect. Dis. 57, 1520.

Ito, T., Couceiro, J.N., Kelm, S., Baum, L.G., Krauss, S., Castrucci, M.R., I. Donatelli, Kida, H., Paulson, J.C., Webster, R.G., and Kawaoka, Y. 1998. Molecular basis for the generation in pigs of influenza A viruses with pandemic potential. J. Virol. 72, 7367-7373.

Ito, T., Goto, H., Yamamoto, E., Tanaka, H., Takeuchi, M., Kuwayama, M., Kawaoka, Y., and Otsuki, K. 2001. Generation of a highly pathogenic avian influenza A virus from an avirulent field isolate by passaging in chickens. J. Virol. 75, 4439-4443.

Jahrling, P.B., Geisbert, T.W., Dalgard, D.W., Johnson, E.D., Ksiazek, T.G., Hall,W.C., and Peters, C.J. 1990. Preliminary report: Isolation of Ebola virus from monkeys imported to USA. Lancet 335, 502-505.

Jennings, L.C., and Miles, J.A. 1978. A study of acute respiratory disease in the community of Port Chalmers. II. Influenza A/Port Chalmers/1/73: Intrafamilial spread and the effect of antibodies to the surface antigens. J. Hyg. (Lond.) 81, 67-75.

Jezek, Z., Kriz, B., and Rothbauer, V. 1983. Camelpox and its risk to the human population. J. Hyg. Epidemiol. Microbiol. Immunol. 27, 29-42.

Jezek, Z., Arita, I., Mutombo, M., Dunn, C., Nakano, J.H., and Szczeniowski, M. 1986. Four generations of probable person-to-person transmission of human monkeypox. Am. Epidemiol, J. 123, 10041012.

Johnson, J.R., Kuskowski, M.A., Smith, K., O'Bryan, T.T., and Tatini, S. 2005. Antimicrobial-resistant and extraintestinal pathogenic Escherichia coli in retail foods J. Infect. Dis. 191, 1040-1049.

Johnson, J.R., and Stell, A.L. 2000. Extended virulence genotypes of Escherichia coli strains from patients with urosepsis in relation to phylogeny and host compromise. J. Infect. Dis. 181, 261272.

Johnson, N.P.A.S., and Mueller, J. 2002. Updating the accounts: Global mortality of the 1918-1920 "Spanish" influenza pandemic. Bull. Hist. Med. 76, 105-115.

Johnson, R.T. 2003. Emerging viral infections of the nervous system. J. Neurovirol. 9, 140-147.

Johnson, T.J., Kariyawasam, S., Wannemuehler, Y., Mangiamele, P., Johnson, S.J., Doetkott, C., Skyberg, J.A., Lynne, A.M., Johnson, J.R., and Nolan, L.K. 2007. The genome sequence of avian pathogenic Escherichia coli strain $\mathrm{O} 1: \mathrm{K} 1: \mathrm{H} 7$ shares strong similarities with human extraintestinal pathogenic E. coli genomes. J. Bacteriol. 189, 3228-3236.

Joint United Nations Programme on HIVIAIDS andWorld Health Organization (UNAIDS \& WHO). 2005. AIDS epidemic update: December 2005.

Joint United Nations Programme on HIVIAIDS (UNAIDS). 2006. 2006 report on the global AIDS epidemic. Geneva, Switzerland: UNAIDS.

Jones, D.S. 2004. Rationalizing Epidemics: Meanings and Uses of American Indian Mortality Since 1600. Cambridge, MA: Harvard University Press.

Jones, P.W., Collins, P., Brown, G.T., and Aitken, M.M. 1983. Salmonella saint-paul infection in two dairy herds. J. Hyg. (Lond). 91, 243-257.

Jones, T., Donnelly, C.A., and Stamp Dawkins, M. 2005. Environmental and management factors affecting the welfare of chickens on commercial farms in the United Kingdom and Denmark stocked at five densities. Poult. Sci. 84, 1155-1165.

Jones-Engel, L., Engel, G.A., Heidrich, J., Chalise, M., Poudel, N., Viscidi, R., Barry, P.A., Allan, J.S., Grant, R., and Kyes, R. 2006. Temple monkeys and health implications of commensalism, Kathmandu, Nepal. Emerg. Infect. Dis. 12, 900-906. 
Jones-Engel, L., Engel, G.A., Schillaci, M.A., Rompis, A., Putra, A., Suaryana, K.G., Fuentes, A., Beer, B., Hicks, S., White, R., Wilson, B., and Allan, J.S. 2005. Primate-to-human retroviral transmission in Asia. Emerg. Infect. Dis. 11, 1028-1035.

Joy, D.A., Feng, X., Mu, J., Furuya, T., Chotivanich, K., Krettli, A.U., Ho, M., Wang, A., White, N.J., Suh, E., Beerli, P., and Su, X.Z. 2003. Early origin and recent expansion of Plasmodium falciparum. Science 300, 318-321.

Kan, B., Wang, M., Jing, H., Xu, H., Jiang, X., Yan, M., Liang, W., Zheng, H., Wan, K., Liu, Q., Cui, B., Xu, Y., Zhang, E., Wang, H., Ye, J., Li, G., Li, M., Cui, Z., Qi, X., Chen, K., Du, L., Gao, K., Zhao, Y.T., Zou, X.Z., Feng, Y.J., Gao, Y.F., Hai, R., Yu, D., Guan, Y., and Xu, J. 2005. Molecular evolution analysis and geographic investigation of severe acute respiratory syndrome coronavirus-like virus in palm civets at an animal market and on farms. J. Virol. 79, 1189211900.

Kaplan, M.M., and Webster, R.G. 1977. The epidemiology of influenza. Sci. Am. 237, 88-106.

Karesh, W., and Cook, R.A. 2005. The human-animal link. Foreign Aff. 84, 38-50.

Karesh,W.B., Cook, R.A., Bennett, E.L., and Newcomb, J. 2005.Wildlife trade and global disease. Emerg. Infect. Dis. 11, 1000-1002.

Karmali, M.A, Steele, B.T., Petric, M., and Lim, C. 1983. Sporadic cases of haemolytic-uraemic syndrome associated with faecal cytotoxin and cytotoxin-producing Escherichia coli in stools. Lancet 1, 619-620.

Karpowicz, P., Cohen, C.B., and van der Kooy, D. 2004. Is it ethical to transplant human stem cells into nonhuman embryos? Nature Med. 10, 331-335.

Kawaoka, Y., Naeve, C.W., and Webster, R.G. 1984. Is virulence of H5N2 influenza viruses in chickens associated with loss of carbohydrate from the hemagglutinin? Virology 139, 303-316.

Kawaoka, Y., and Webster, R.G. 1985. Evolution of the A/Chicken/Pennsylvania/83 (H5N2) influenza virus. Virology 146, 130-137.

Kay, R. 1991. The site of the lesion causing hearing loss in bacterial meningitis: A study of experimental streptococcal meningitis in guinea-pigs. Neuropathol. Appl. Neurobiol. 17, 485-493.

Keawcharoen, J., Oraveerakul, K., Kuiken, T., Fouchier, R.A., Amonsin, A., Payungporn, S., Noppornpanth, S.,Wattanodorn, S., Theambooniers, A., Tantilertcharoen, R., Pattanarangsan, R., Arya, N., Ratanakorn, P., Osterhaus, D.M., and Poovorawan, Y. 2004. Avian influenza H5N1 in tigers and leopards. Emerg. Infect. Dis. 10, 2189-2191.

Kida,H.,Yanagawa,R., andMatsuoka,Y. 1980.Duck influenza lacking evidence of disease signs and immune response. Infect. Immun. 30, 547-553.

Kido, H., Okumura, Y., Yamada, H., Le, T.Q., and Yano, M. 2007. Proteases essential for human influenza virus entry into cells and their inhibitors as potential therapeutic agents. Curr. Pharm. Des. 13, 403-412.

Kim, E.H., and Aoki, T. 1993. Drug resistance and broad geographical distribution of identical R plasmids of Pasteurella piscicida isolated from cultured yellowtail in Japan. Microbiol. Immunol. 37, 103109.

Kinney, K.S., Austin, C.E., Morton, D.S., and Sonnenfeld, G. 2000. Norepinephrine as a growth stimulating factor in bacteria: Mechanistic studies. Life Sci. 67, 3075-3085.

Kistemann, T., Zimmer, S., Vagsholm, I., and Andersson, Y. 2004. GIS-supported investigation of human EHEC and cattle VTEC 0157 infections in Sweden: Geographical distribution, spatial variation and possible risk factors. Epidemiol. Infect. 132, 495-505.

Kite-Powell, H. 2004. Down on the farm, raising fish. Oceanus, September 21.

Klasing, K.C., Laurin, D.E., Peng, R.K., and Fry, D.M. 1987. Immunologically mediated growth depression in chicks: Influence of feed intake, corticosterone and Interleukin-1. J. Nutr. 117, 1629-1637.

Klatt, E.C.,Pavlova, Z.,Teberg, A.J., andYonekura, M.L. 1986. Epidemic neonatal listeriosis at autopsy. Hum. Pathol. 17, 1278-1281.

Klopfleisch, R., Wolf, P.U., Wolf, C., Harder, T., Starick, E., Niebuhr, M., Mettenleiter, T.C., and Teifke, J.P. 2007. Encephalitis in a stone marten (Martes foina) after natural infection with highly pathogenic avian influenza virus subtype H5N1. J. Comp. Pathol. Aug 7; [Epub ahead of print].

Knipe, D.M., and Howley, P.M., eds. 2007. Fields Virology, 5th ed. Philadelphia, PA: Lippincott, Williams, \& Wilkins.

Knowles, T.G., and Broom, D.M. 1993. Effect of catching method on the concentration of plasma corticosterone in end-of-lay battery hens. Vet. Rec. 133, 527-528. 
Knox, W.A., Galbraith, N.C., Lewis, M.J., Hickie, G.C., and Johnston, H.H. 1963. A milkborne outbreak of food poisoning due to Salmonella Heidelberg. J. Hyg. 61, 175-185.

Kohler, B., Karch, H., and Schmidt, H. 2000. Antibacterials that are used as growth promoters in animal husbandry can affect the release of Shiga-toxin-2-converting bacteriophages and Shiga toxin 2 from Escherichia coli strains. Microbiology 146, 1085-1090.

Kristensson, K. 2006. Avian influenza and the brain: Comments on the occasion of resurrection of the Spanish flu virus. Brain Res. Bull. 68, 406-413.

Kruse, H., and Sorum, H. 1994. Transfer of multiple drug resistance plasmids between bacteria of diverse origins in natural microenvironments. Appl. Environ. Microbiol. 60, 4015-4021.

Kuehn, B.M. 2005. New human retroviruses discovered: Evidence that cross-species leap not a rare event. JAMA 293, 2989-2990.

Kuriyama, T., Machida, K., and Suzuki, K. 1996. Importance of correlations between phagocytic activity and superoxide production of neutrophils under conditions of voluntary exercise and stress. $J$. Clin. Lab. Anal. 10, 458-464.

Kurtz, J., Manvell, R.J., and Banks, J. 1996. Avian influenza virus isolated from a woman with conjunctivitis. Lancet 348, 901-902.

Labadie, K., Dos Santos Afonso, E., Rameix-Welti, M.A., van derWerf, S., and Naffakh, N. 2007. Hostrange determinants on the PB2 protein of influenza $A$ viruses control the interaction between the viral polymerase and nucleoprotein in human cells. Virology 362, 271-282.

Lam, S.K. 2003. Nipah virus: A potential agent of bioterrorism? Antiviral Res. 57, 113-119.

Lammerding, A M., and Doyle, M.P. 1990. Stability of Listeria monocytogenes to non-thermal processing conditions. In Foodborne listeriosis, eds. Miller, A.J., Smith, J.L., and Somkuti, G.A., 195-202. New York, NY: Elsevier.

Lanada, E.B., Morris, R.S., Jackson, R., and Fenwick, S.G. 2005. Prevalence of Yersinia species in goat flocks. Aust. Vet. J. 83, 563-566.

Lanciotti, R.S., Roehrig, J.T., Deubel, V., Smith, J., Parker, M., Steele, K., Crise, B., Volpe, K.E., Crabtree, M.B., Scherret, J.H., Hall, R.A., MacKenzie, J.S., Cropp, C.B., Panigrahy, B., Ostlund, E., Schmitt, B., Malkinson, M., Banet, C., Weissman, J., Komar, N., Savage, H.M., Stone, W., McNamara, T., and Gubler, D.J. 1999. Origin of the West Nile virus responsible for an outbreak of encephalitis in the northeastern United States. Science 286, 2333-2337.

Larkin, M. 2000. Hunting and logging linked to emerging infectious diseases. Lancet 356, 1173.

Lashley, F.R. 2004. Emerging infectious diseases: Vulnerabilities, contributing factors and approaches. Exp. Rev. Anti-infect. Ther. 2, 299-316.

Lasley, F.A. 1987. Economics of avian influenza. Proceedings of the 2nd International Symposium on Avian Influenza, 390-399. Athens, GA: U.S. Animal Health Association.

Laurent, S., Esnault, E., Dambrine, G., Goudeau, A., Choudat, D., and Rasschaert, D. 2001. Detection of avian oncogenic Marek's disease herpesvirus DNA in human sera. J. Gen. Virol. 82, 233-240.

Laver,W.G., andWebster, R.G. 1972. Antibodies to human influenzavirus neuraminidase (the A-Asian-57 H2N2 strain) in sera from Australian pelagic birds. Bull World Health Organ. 47, 535-541.

Lawrie, M. 2004. Animal welfare gains from avian influenza? Aust. Vet. J. 82, 135.

Lecuit, M., Abachin, E., Martin, A., Poyart, C., Pochart, P., Suarez, F., Bengoufa, D., Feuillard, J., Lavergne, A., Gordon, J.I., Berche, P., Guillevin, L., and Lortholary, O. 2004. Immunoproliferative small intestinal disease associated with Campylobacter jejuni. N. Engl. J. Med. 350, 239-248.

Lederberg, J., Shope, R.E., and Oaks, S.C. 1992. Emerging Infections: Microbial Threats to Health in the United States. Washington, D.C.: National Academies Press, 15.

Lee, H., Kim, S., Kang, M., Kim, W., and Cho, B. 1998. Prevalence of human foamy virus-related sequences in the Korean population. J. Biomed. Sci. 5, 267-273.

Lee, J.W., and McKibbin, W. 2003. Globalization and disease: The case of SARS.Working Paper No. 2003/16. Canberra, Australia: Research School of Pacific and Asian Studies, Australian National University, and Washington, D.C: The Brookings Institution.

Lee, M.S., Chang, P.C., Shien, J.H., Cheng, M.C., Chen, C.L., and Shieh, H.K. 2006. Genetic and pathogenic characterization of H6N1 avian influenza viruses isolated in Taiwan between 1972 and 2005. Avian Dis. 50, 561-571.

Lee, P.J., and Krilov, L.R. 2005. When animal viruses attack: SARS and avian influenza. Ped. Ann. 34, 43-52. 
Legrand, J., Grais, R.F., Boelle, P.Y., Valleron, A.J., and Flahault, A. 2007. Understanding the dynamics of Ebola epidemics. Epidemiol. Infect. 135, 610-621.

Lehane, L., and Rawlin, G.T. 2000. Topically acquired bacterial zoonoses from fish: A review. Med. J. Aust. 173, 256-259.

Lehmann, N.I., and Gust, I.D. 1971. Viraemia in influenza: A report of two cases. Med. J. Aust. 2, 11661169.

Li, S., Orlich, M.A., and Rott, R. 1990. Generation of seal influenza virus variants pathogenic for chickens, because of hemagglutinin cleavage site changes. J. Virol. 64, 3297-3303.

Li, W., Shi, Z., Yu, M., Ren, W., Smith, C., Epstein, J.H., Wang, H., Crameri, G., Hu, Z., Zhang, H., Zhang, J., McEachern, J., Field, H., Daszak, P., Eaton, B.T., S. Zhang, and Wang, L. 2005. Bats are natural reservoirs of SARS-like coronaviruses. Science 310, 676-679.

Liang, A.P. 2002. Current state of foodborne illness. Thinking globally-working locally: A conference on food safety education, September 18-20, Orlando, Florida.

Lin, Y.P., Shaw, M., Gregory, V., Cameron, K., Lim,W., Klimov, A., Subbarao, K., Guan, Y., Krauss, S., Shortridge, K., Webster, R., Cox, N., and Hay, A. 2000. Avian-to-human transmission of H9N2 subtype influenza A viruses: Relationship between H9N2 and H5N1 human isolates. Proc. Natl. Acad. Sci. USA 97, 9654-9658.

Liu, M., Guan, Y., Peiris, M., He, S.,Webby, R.J., Perez, D., andWebster, R.G. 2003. The quest of influenza A viruses for new hosts. Avian Dis. Avian Dis. 47, 849-856.

Liu, W.T., Kao, K.P., Liu, Y.C., and Chang, K.S. 1996. Human foamy virus genome in the thymus of myasthenia gravis patients. Chung Hua Min Kuo Wei Sheng Wu Chi Mien I Hsueh Tsa Chih 29, 162-165.

Loehr, R.C. 1978. Hazardous solid waste from agriculture. Environ. Health Perspect. 27, 261-273.

Lorber, B. 1996. Listeriosis. Clin. Infect. Dis. 24, 1-11.

Lord Phillips of Worth Maltravers, Bridgeman, J., and M. Ferguson-Smith. 2000. The BSE Inquiry. London: The Stationery Office.

Lu, X., Tumpey, T.M., Morken, T., Zaki, S.R., Cox, N.J., and Katz, J.M. 1999. A mouse model for the evaluation of pathogenesis and immunity to influenza A (H5N1) viruses isolated from humans. $J$. Virol. 73, 5903-5911.

Luby, S.P., Rahman, M., Hossain, M.J., Blum, L.S., Husain, M.M., Gurley, E., Khan, R., Ahmed, B.N., Rahman, S., Nahar, N., Kenah, E., Comer, J.A., and Ksiazek, T.G. 2006. Foodborne transmission of Nipah virus, Bangladesh. Emerg. Infect. Dis. 12, 1888-1894.

Ludwig, B., Kraus, F.B., Allwinn, R., Doerr, H.W., and Preiser, W. 2003. Viral zoonoses: A threat under control? Intervirology 46, 71-78.

Lun, Z.R.,Wang, Q.P., Chen, X.G., Li, A.X., and Zhu, X.Q. 2007. Streptococcus suis: An emerging zoonotic pathogen. Lancet Infect. Dis. 7, 201-209.

Lynch, M., Daly, M., O'Brien, B., Morrison, F., Cryan, B., and Fanning, S. 1999. Salmonella tel-el-kebir and terrapins. J. Infect. 38, 182-184.

Lyte, M. 2004. The biogenic amine tyramine modulates the adherence of Escherichia coli $\mathrm{O} 157: \mathrm{H} 7$ to intestinal mucosa. J. Food Prot. 67, 878-883.

Mack, T.M. 2005. The ghost of pandemics past. Lancet 365, 1370-1372.

MacKenzie, J.S., Drury, P., Ellis, A., Grein, T., Leitmeyer, K.C., Mardel, S., Merianos, A., Olowokure, B., Roth, C., Slattery, R., Thomson, G., Werker, D., and M. Ryan. 2004. The WHO response to SARS and preparations for the future. In Learning from SARS, preparing for the next disease outbreak, workshop summary, eds. Knobler, S., Mahmoud, A., Lemon, S., Mack, A., Sivitz, L., and Oberholtzer, K., 42-49.Washington, D.C.: National Academies Press.

MacKenzie, W.R., Hoxie, N.J., Proctor, M.E., Gradus, M.S., Blair, K.A., D.E Peterson, Kazmierczak, J.J., Addiss, D.G., Fox, K.R., Rose, J.B., and Davis, J.P. 1994. A massive outbreak in Milwaukee of Cryptosporidium infection transmitted through the public water supply. N. Engl. J. Med. 331, 161167.

Mackinnon, M.J., and Read, A.F. 1999. Selection for high and low virulence in the malaria parasite Plasmodium chabaudi. Proc. Biol. Sci. 266, 741-748.

Madec, F., and Rose, N. 2003. How husbandry practices may contribute to the course of infectious diseases in pigs. In 4th international symposium on emerging and re-emerging pig diseases, June 29-July 2, Rome, Italy, 9-18. 
Madelin, T.M., and Wathes, C.M. 1989. Air hygiene in a broiler house: Comparison of deep litter with raised netting floors. Br. Poult. Sci. 30, 23-37.

Maes, D., Deluyker, H., Verdonck, M., Castryck, F., Miry, C., Vrijens, B., and de Kruif, A. 2000. Herd factors associated with the seroprevalences of four major respiratory pathogens in slaughter pigs from farrow-to-finish pig herds. Vet. Res. 31, 313-327.

Mahieux, R., and Gessain, A. 2003. HTLV-1 and associated adult T-cell leukemia/lymphoma. Rev. Clin. Exp. Hematol. 7, 336-361.

Mahy, B.W., and Brown, C.C. 2000. Emerging zoonoses: Crossing the species barrier. Rev. Sci. Tech. Off. Int. Epiz. 1, 33-40.

Maines,T.R., Chen, L.M., Matsuoka,Y., Chen, H.,Rowe,T., Ortin, J., Falcon, A., Hien, N.T., Mai, L.Q., Sedyaningsih, E.R., Harun, S., Tumpey, T.M., Donis, R.O., N.J. Cox, Subbarao, K., and Katz, J.M. 2006. Lack of transmission of H5N1 avian-human reassortant influenza viruses in a ferret model. Proc. Natl. Acad. Sci. USA 10 3, 12121-12126.

Maines, T.R., Lu, X.H., Erb, S.M., Edwards, L., Guarner, J., Greer, P.W., Nguyen, D.C., Szretter, K.J., Chen, L.M., Thawatsupha, P., Chittaganpitch, M., Waicharoen, S., Nguyen, D.T., Nguyen, T., Nguyen, H.H., Kim, J.H., Hoang, L.T., Kang, C., L.S. Phuong, Lim, W., Zaki, S., Donis, R.O., Cox, N.J., Katz, J.M., and Tumpey, T.M. 2005. Avian influenza (H5N1) viruses isolated from humans in Asia in 2004 exhibit increased virulence in mammals. J. Virol. 79, 11788-11800.

Mangel, M., and Stamps, J. 2001. Trade-offs between growth and mortality and the maintenance of individual variation in growth. Evol. Ecol. Res. 3, 583-593.

Mantovani, A. 2001. Notes on the development of the concept of zoonoses. WHO Mediterranean Zoonoses Control Centre Information Circular 51.

Marangon, S., Capua, S.I., Rossi, E., Ferre, N., M. Dalla Pozza, Bonfanti, L., and Manelli, A. 2003. The control of avian influenza in areas at risk: The Italian experience 1997. In Proceedings of the FrontisWorkshop on Avian Influenza: Prevention and Control, eds. Schrijver, R.S. andKoch, G., October 13-15, The Netherlands. <library.wur.nl/frontis/avian influenza/05 marangon.pdf>.

Mararova, N.V., Ozaki, H., Kida, H., Webster, R.G., and Perez, D.R. 2003. Replication and transmission of influenza viruses in Japanese quail. Virology 310, 8-15.

Marg, H., Scholz, H.C., Arnold, T., Rosler, U., and Hensel, A. 2001. Influence of long-time transportation stress on reactivation of Salmonella Typhimurium DT104 in experimentally infected pigs. Berlin.Munch. Tierarztl.Wochenschr. 114, 385-388.

Marks, G., and Beatty,W.K. 1976. Epidemics.NewYork,NY: Charles Scribner's Sons.

Markwell, D.D., and Shortridge, K.F. 1982. Possible waterborne transmission and maintenance of influenza viruses in domestic ducks. Appl. Environ. Microbiol. 43, 110-115.

Martens, W.J., Niessen, L.W., Rotmans, J., Jetten, T.H., and McMichael, A.J. 1995. Potential impact of global climate change on malaria risk. Environ. Health Perspect. 10 3, 458-464.

Martini, G.A. 1969. Marburg agent disease in man. Trans. R. Soc. Trop. Med. Hyg. 63, 295-302.

Marx, P.A., Alcabes, P.G., and Drucker, E. 2001. Serial human passage of simian immunodeficiency virus by unsterile injections and the emergence of epidemic human immunodeficiency virus in Africa. Philos. Trans. R. Soc. Lond. B. Biol. Sci. 356, 911-920.

Mase, M., Eto, M., Tanimuraa, N., Imaic, K., Tsukamotoa, K., Horimoto, T., Y. Kawaoka, and Yamaguchi, S. 2005. Isolation of a genotypically unique H5N1 influenza virus from duck meat imported into Japan from China.Virology 339, 101-109. <depts.washington.edu/einet/?a=printArticle\&print=649>.

Mason, L.L., and Crawford, R.D. 1993. Appendix A: Global status of livestock and poultry species. In Managing global genetic resources, 141-170. Washington, D.C.: National Academies Press.

Massin, P., van der Werf, S., and Naffakh, N. 2001. Residue 627 of PB2 is a determinant of cold sensitivity in RNA replication of avian influenza viruses. J. Virol. 75, 5398-5404.

Matrosovich, M.N., Matrosovich, T.Y., Gray, T., Roberts, N.A., and Klenk, H.D. 2004. Human and avian influenza viruses target different cell types in cultures of human airway epithelium. Proc. Natl. Acad. Sci. USA 10 1, 4620-4624.

Matrosovich, M., Suzuki, T., Hirabayashi, Y., Garten, W., Webster, R.G., and Klenk, H.D. 2006. Gangliosides are not essential for influenza virus infection. Glycoconj. J. 23, 107-113.

Maupin, G.O., Fish, D., Zultowsky, J., Campos, E.G., and Piesman, J. 1991. Landscape ecology of Lyme disease in a residential area of Westchester County, New York. Am. J. Epidemiol. 133, 11051113. 
Mayaux, P., Holmgren, P., Achard, F., Eva, H., Stibig, H.J., and Branthomme, A. 2005. Tropical forest cover change in the 1990s and options for future monitoring. Philos. Trans. R. Soc. Lond. B. Biol. Sci. 360, 373-384.

McCall, S., Henry, J.M., Reid, A.H., and Taubenberger, J.K. 2001. Influenza RNA not detected in archival brain tissues from acute encephalitis lethargica cases or in postencephalitic Parkinson cases. $J$. Neuropathol. Exp. Neurol. 60, 696-704.

McChesney, D.G., Kaplan, G., and Gardner, P. 1995. FDA survey determines Salmonella contamination. Feedstuffs 67, 20-23.

McDowell, R.M., and McElvaine, M.D. 1997. Long-term sequelae to foodborne disease. Rev. Sci. Tech. Off. Int. Epiz. 16, 337-341.

McGirk, T., Adiga, A., and Glacier, S. 2005.Will the next pandemic rival 1918? Times Asia, July 4.

McGowan Jr., J.E. 2001. Economic impact of antimicrobial resistance. Emerg. Infect. Dis. 7, 286-292.

McKibbin, W.J., and A.A Sidorenko. 2006. Global macroeconomic consequences of pandemic influenza. Sydney, Australia: Lowy Institute for International Policy.

McLeod, A.,Morgan, N., Prakash, A., and Hinrichs, J. 2005. Economic and social impact of avian influenza. Proceedings of the joint FAO/OMS/OIE/WorldBank conference on avian influenza and human pandemic influenza, November 7-9, Geneva.

McMichael, A.J. 2004. Environmental and social influences on emerging infectious diseases: Past, present and future. Philos. Trans. R. Soc. Lond. B. Biol. Sci. 359, 1049-1058.

McMichael,T. 2001a. HumanFrontiers, Environments and Disease. Cambridge, UK: Cambridge University Press.

McMichael, T. 2001b. Transitions in human health: Surviving this millennium by learning from the past one hundred millennia. Glob. Change Hum. Health 2, 76-77.

McNamara, T.S. 2002. Diagnosis and control of zoonotic infections: Pathology and early recognition of zoonotic disease outbreaks. In The emergence of zoonotic diseases: Understanding the impact on animal and human health-workshop summary, 64-78. Washington, D.C.: National Academies Press.

Mead, P.S., Slutsker, L., Dietz,V., McCaig, L.F., Bresee, J.S., Shapiro, C., Griffin, P.M., and Tauxe, R.V. 1999. Food-related illness and death in the United States. Emerg. Infect. Dis. 5, 607-625.

Meiering, C.D., and Linial, M.L. 2001. Historical perspective of foamy virus epidemiology and infection. Clin. Microbiol. Rev. 14, 165-176.

Mellon, M.G., Benbrook, C., and Benbrook, K.L. 2001. Hogging It! Estimates of Antimicrobial Abuse in Livestock. Cambridge, MA: Union of Concerned Scientists.

Melville, D.S., and Shortridge, K.F. 2004. Influenza: Time to come to grips with the avian dimension. Lancet Infect. Dis. 4, 261-262.

Mench, J.A. 2002. Consumer voices, dollars are changing animal welfare standards. University of California-Davis, Sustainable Agriculture Newsletter 14(2). <sarep.ucdavis.edu/newsltr/v14n2/sa-1.htm>.

Mench, J., and Keeling, L. 2001. The social behaviour of domestic birds. In Social behaviour in farm animals, eds. Keeling, L.J. and Gonyou, H.W., 177-211. Wallingford, UK: CAB International.

Meng, J. 2002. China's challenge: Employment and unemployment. Research Institute of Economy, Trade and Industry.

Menge, C., Blessenohl, M., Eisenberg, T., Stamm, I., and Baljer, G. 2004. Bovine ileal intraepithelial lymphocytes represent target cells for Shiga toxin 1 from Escherichia coli. Infect. Immun. 72, $1896-1905$.

Meredith, M. 2004. Zoonotic disease risks: 2004 update. American Association of Swine Veterinarians.

Merson, M.H. 2006. The HIV-AIDS pandemic at 25, The global response. N. Engl. J. Med. 354, 24142417.

Meslin, F.X., Stohr, K., and Heymann, D. 2000. Public health implications of emerging zoonoses. Rev. Sci. Tech. Off. Int. Epiz. 19, 310-317.

Messenger, S.L., Molineux, I.J., and Bull, J.J. 1999. Virulence evolution in a virus obeys a trade-off. Proc. Biol. Sci. 266, 397-404.

Michino, H., Araki, K., Minami, S., Takaya, S., Sakai, N., Miyazaki, M., Ono, A., and Yanagawa, H. 1999. Massive outbreak of Escherichia coli O157:H7 infection in schoolchildren in Sakai City, Japan, associated with consumption of white radish sprouts. Am. J. Epidemiol. 150, 787-796. 
Middleton, D., Bingham, J., Selleck, P., Lowther, S., Gleeson, L., Lehrbach, P., Robinson, S., Rodenberg, J., Kumar, M., and Andrew, M. 2007. Efficacy of inactivated vaccines againstH5N1 avian influenza infection in ducks. Virology 359, 66-71.

Miller, M.M., and Schat, K.A. 2004. Chicken infectious anemia virus: An example of the ultimate hostparasite relationship. Avian Dis. 48, 734-745.

Mills, C.E., Robins, J.M., and Lipsitch, M. 2004. Transmissibility of 1918 pandemic influenza. Nature 432, 904-906.

Mo, I.P., Brugh, M., Fletcher, O.J., Rowland, G.N., and Swayne, D.E. 1997a. Comparative pathology of chickens experimentally inoculated with avian influenza viruses of low and high pathogenicity. Avian Dis. 41, 125-136.

Mo, I.P., Song, C.S., Kim, K.S., and Rhee, J.C. 1997b. An occurrence of non-highly pathogenic avian influenza in Korea. In Proceedings of the Fourth International Symposium on Avian Influenza, eds. Swayne, D. and Slemons, R., 379-383. Tallahassee, FL: U.S. Animal Health Association.

Moce-Llivina, L., Muniesa, M., Pimenta-Vale, H., Lucena, F., and Jofre, J. 2003. Survival of bacterial indicator species and bacteriophages after thermal treatment of sludge and sewage. Appl. Environ. Microbiol. 69, 1452-1456.

Mohd Nor, M.N., Gan, C.H., and Ong, B.L. 2000. Nipah virus infection of pigs in peninsular Malaysia. Rev. Sci. Tech. Off. Int. Epiz. 19, 160-165.

Monath, T.P. 1999. Ecology of Marburg and Ebola viruses: Speculations and directions for future research. J. Infect. Dis. 179 Suppl 1, S127-S138.

Moore, J.E., Barton, M.D., Blair, I.S., Corcoran, D., Dooley, J.S., Fanning, S., Kempf, I., Lastovica, A.J., Lowery, C.J., Matsuda, M., McDowell, D.A., McMahon, A., Millar, B.C., Rao, J.R., Rooney, P.J., Seal, B.S., Snelling,W.J., and Tolba, O. 2006. The epidemiology of antibiotic resistance in Campylobacter. Microb. Infect. 8, 1955-1966.

Moore, J.E., and Matsuda, M. 2002. The history of Campylobacter: Taxonomy and nomenclature. Irish Vet. J. 10, 495-501.

Morens, D.M., Folkers, G.K., and Fauci, A.S. 2004. The challenge of emerging and re-emerging infectious diseases. Nature 430, 242-249.

Morishima, T., Togashi, T., Yokota, S., Okuno, Y., Miyazaki, C., Tashiro, M., and Okabe, N. 2002. Encephalitis and encephalopathy associated with an influenza epidemic in Japan. Clin. Infect. Dis. 35, 512-517.

Moroney, J.F., Guevara, R., Iverson, C., Chen, F.M., Skelton, S.K., Messmer, T.O., Plikaytis, B.,Williams, P.O., Blake, P., and Butler, J.C. 1998. Detection of chlamydiosis in a shipment of pet birds, leading to recognition of an outbreak of clinically mild psittacosis in humans. Clin. Infect. Dis. 26, $1425-1429$.

Moulin-Schouleur, M., Reperant, M., Laurent, S., Bree, A., S. Mignon-Grasteau, Germon, P., Rasschaert, D., and Schouler, C. 2007. Extra-intestinal pathogenic Escherichia coli of avian and human origin: Link between phylogenetic relationships and common virulence patterns. J. Clin. Microbiol, Jul 25; [Epub ahead of print].

Muir, W.M., and Aggrey, S.E. 2003. Introductory editorial: Breeding for disease resistance in its evolutionary context. In Poultry genetics, breeding and biotechnology, ix-xiv. Oxfordshire, UK: CAB International.

Munster, V.J., de Wit, E., van Riel, D., Beyer, W.E., Rimmelzwaan, G.F., Osterhaus, A.D., Kuiken, T., and Fouchier, R.A. 2007. The molecular basis of the pathogenicity of the Dutch highly pathogenic human influenza A H7N7 viruses. J. Infect. Dis. 196, 258-265.

Murphy, B. 1993. Factors restraining emergence of new influenza viruses. In Emerging viruses, ed. Morse, S.S., 234-240. New York, NY: Oxford University Press.

Murphy, B.R., Hinshaw, V.S., Sly, D.L., London, W.T., Hosier, N.T., Wood, F.T.,Webster, R.G., and Chanock, R.M. 1982. Virulence of avian influenza A viruses for squirrel monkeys. Infect. Immun. $37,1119-1126$.

Murphy, F.A. 1999. Emerging zoonoses. Emerg. Infect. Dis. 4, 429-435.

Murray, C.J., Lopez, A.D., Chin, B., Feehan, D., and Hill, K.H. 2006. Estimation of potential global pandemic influenza mortality on the basis of vital registry data from the 1918-20 pandemic: A quantitative analysis. Lancet 368, 2211-2218. 
Murray, E.G.D., Webb, R.A., and Swann, M.B.R. 1926. A disease of rabbits characterised by a large mononuclear leukocytosis, caused by a hitherto undescribed bacillus Bacterium monocytogenes (n. sp.). J. Pathol. Bacteriol. 29, 407-439.

Myers, K.P., Olsen, C.W., Setterquist, S.F., Capuano, A.W., Donham, K.J., E.L. Thacker, Merchant, J.A., and Gray, G.C. 2006. Are swine workers in the United States at increased risk of infection with zoonotic influenza virus? Clin. Infect. Dis. 42, 14-20.

Myint, K.S., and Gibbons, R.V. 2007. Hepatitis E: A neglected threat. Trans. R. Soc. Trop. Med. Hyg.; July 19 [Epub ahead of print].

Naeem, K., Siddique, N., Ayaz, M., and Jalalee, M.A. 2007. Avian influenza in Pakistan: Outbreaks of low- and high-pathogenicity avian influenza in Pakistan during 2003-2006. Avian Dis. 51, 189193.

Naeve, C.W., Hinshaw, V.S., and Webster, R.G. 1984. Mutations in the hemagglutinin receptor-binding site can change the biological properties of an influenza virus. J. Virol. 51, 567-569.

Nair, V. 2005. Evolution of Marek's disease: A paradigm for incessant race between the pathogen and the host. Vet. J. 170, 175-183.

Najera, J.A. 1989. Malaria and thework of the WHO. Bull.World Health Organ. 67, 229-243.

Nansen, P., Foldager, J., Hansen, J.W., Henriksen, S.A., and Jørgensen, R.J. 1988. Grazing pressure and acquisition of O. ostertagi in calves. Vet. Parasitol. 27, 325-335.

Narain, J.P., and Lofgren, J.P. 1989. Epidemic of restaurant-associated illness due to Salmonella newport. South. Med. J. 82, 837-840.

Narayan, O., Lang, G., and Rouse, S.T. 1969. A new influenza A virus infection in turkeys. IV Experimental susceptibility of domestic birds to virus strain ty/Ontario/7732/1966. Archiv. Gesamte. Virusforsch. 26, 149-165.

National Renderers Association (NRA). 2005. Production, U.S., Consumption and Export of Rendered Products, 1998-2003.

National Turkey Federation (NTF). 1995. Meat bird production/growout. Food safety best management practices for the production of turkeys, December.

Nelson, J.M., Chiller, T.M., Powers, J.H., and Angulo, F.J. 2007. Fluoroquinolone-resistant Campylobacter species and the withdrawal of fluoroquinolones from use in poultry: A public health success story. Clin. Infect. Dis. 44, 977-980.

Nestle, M. 2003. Safe Food: Bacteria, Biotechnology, and Bioterrorism. Berkeley and Los Angeles, CA: University of California Press.

Newman, S.H., Epstein, J.H., and Schloegel, L.M. 2005. The nature of emerging zoonotic diseases: Ecology, prediction, and prevention. Med. Lab. Obs. 37, 10-19.

Nguyen-Van-Tam, J., Nair, P., Acheson, P., Baker, A., Barker, M., Bracebridge, S., Croft, J., Ellis, J., Gelletlie, R., Gent, N., Ibbotson, S., Joseph, C., Mahgoub, H., Monk, P., Reghitt, T.W., Sundkvist, T., Sellwood, C., Simpson, J., Smith, J., J.M. Watson, Zambon, M., and Lightfoot, N. 2006. Outbreak of low pathogenicity H7N3 avian influenza in UK, including associated case of human conjunctivitis. Euro. Surveill. 11, E060504.2.

Nicholls, J.M., Chan, M.C., Chan, W.Y., Wong, H.K., Cheung, C.Y., Kwong, D.L., Wong, M.P., Chui, W.H., Poon, L.L., Tsao, S.W., Guan, Y., and Peiris, J.S. 2007. Tropism of avian influenza A (H5N1) in the upper and lower respiratory tract. Nature Med. 13, 147-149.

Nierenberg, D. 2005. Happier meals: Rethinking the global meat industry. Worldwatch Institute Paper 171.

Nightingale, K.K., Schukken, Y.H., Nightingale, C.R., Fortes, E.D., Ho, A.J., Her, Z., Grohn, Y.T., McDonough, P.L., and Wiedmann, M. 2004. Ecology and transmission of Listeria monocytogenes infecting ruminants and in the farm environment. Appl. Environ. Microbiol. 70, 4458-4467.

Nisbett, R.A., and Monath, T.P. 2001. Viral traffic, transnational companies and logging in Liberia, West Africa. Glob. Change Hum. Health 2, 18-19.

Nishimura, H., Itamura, S., Iwasaki, T., Kurata, T., and Tashiro, M. 2000. Characterization of human influenza A (H5N1) virus infection in mice: Neuro-,pneumo- and adipotropic infection. J. Gen. Virol. 81, 2503-2510.

NN. 1995. Superglue advocated for preventing fecal leakage in poultry. Food Chemical News April 24, 12.

NN. 2004. Virus hitting chicken immunity may be cause of bird flu. eFeedLink, January 28.

NN. 2006. George W, H. Bush christened at Newport News. U.S. Navy Public Affairs Office. October 7. 
NN. 2007a. Fourth case of transfusion-associated vCJD infection in the United Kingdom. Euro. Surveill. 12, E070118.4.

NN. 2007b. National Creutzfeldt-Jakob Disease Surveillance Unit. CJD Statistics. University of Edinburgh, July 10.

Noland, P.R., Ford, B.F., and Ray, M.L. 1955. The use of ground chicken litter as a source of nitrogen for gestating-lactating ewes and fattening steers. J. Anim. Sci. 14, 860-865.

Normile, D. 2005. Infectious diseases: WHO probes deadliness of China's pigborne disease. Science 30 9, 1308-1309.

Norris, K., and Evans, M.R. 2000. Ecological immunology: Life history tradeoffs and immune defense in birds. Behav. Ecol. 11, 19-26.

Nou, X., Rivera-Betancourt, M., Bosilevac, J.M., Wheeler, T.L., Shackelford, S.D., Gwartney, B.L., Reagan, J.O., and Koohmaraie, M. 2003. Effect of chemical dehairing on the prevalence of Escherichia coli $\mathrm{O} 157: \mathrm{H} 7$ and the levels of aerobic bacteria and Enterobacteriaceae on carcasses in a commercial beef processing plant. J. Food Prot. 66, 2005-2009.

Nupp, T.E., and Swihart, R.K. 1996. Effect of forest patch area on population attributes of white-footed mice (Peromyscus leucopus) in fragmented landscapes. Can. J. Zool. 74, 467-472.

Ocepek, M., Pate, M., M. Zolnir-Dovc, and Poljak, M. 2005. Transmission of Mycobacterium tuberculosis from human to cattle. J. Clin. Microbiol. 43, 3555-3557.

Office of Technology Assessment. 1979. Drugs in Livestock Feed: Volume 1 Technical Report. Washington, D.C.: U.S. Government Printing Office.

Olival, K.J., and Daszak, P. 2005. The ecology of emerging neurotropic viruses. J. Neurovirol. 11, 441446.

Omran, A.R. 1971. The epidemiologic transition: A theory of the epidemiology of population change. Milbank Mem. Fund Q. Health Soc. 49, 509-538.

Ostrowski, S.R., Leslie, M.J., Parrott, T., Abelt, S., and Piercy, P.E. 1998. B-virus from pet macaque monkeys: An emerging threat in the United States? Emerg. Infect. Dis. 4, 117-121.

Otte, J., D. Roland-Holst, R. Pfeiffer Soares-Magalhaes, Rushton, J., Graham, J., and Silbergeld, E. 2007. Industrial Livestock Production and Global Health Risks. Food and Agriculture Organization of the United Nations, Pro-Poor Livestock Policy Initiative Research Report.

Oxford, J.S. 2001. The so-called Great Spanish Influenza Pandemic of 1918 may have originated in France in 1916. Philos. Trans. R. Soc. Lond. B. Biol. Sci. 356, 1857-1859.

Oxford, J.S., Lambkin, R., Elliot, A., Daniels, R., Sefton, A., and Gill, D. 2006. Scientific lessons from the first influenza pandemic of the 20th century. Vaccine 24, 6742-6746.

Oxford, J.S., Novelli, P., Sefton, A., and Lambkin, R. 2002. New millennium antivirals against pandemic and epidemic influenza: The neuraminidase inhibitors. Antivir. Chem. Chemother. 13, 205-217.

Padgett, D.A., and Glaser, R. 2003. How stress influences the immune response. Trends Immunol. 24, 444-448.

Page, R.D.M., and Holmes, E. 1998. Molecular Evolution. Oxford, UK: Blackwell Publishing.

Pallares, F.J., Halbur, P.G., Schmitt, C.S., Roth, J.A., Opriessnig, T., P.J. Thomas, Kinyon, J.M.,Murphy, D., Frank, D.E., and Hoffman, L.J. 2003. Comparison of experimental models for Streptococcus suis infection of conventional pigs. Can. J. Vet. Res. 67, 225-228.

Palumbi, S.R. 2001. Humans as the world's greatest evolutionary force. Science 293, 1786-1790.

Pan American Health Organization (PAHO). 2004. Avian influenza virus A (H10N7) circulating among humans in Egypt. Emerg. Infect. Dis. Weekly Updates 2(18), May 7.

Pang, T., Bhutta, Z.A., Finlay, B.B., and Altwegg, M. 1995. Typhoid fever and other salmonellosis: A continuing challenge. TIM 3, 253-255.

Pantin-Jackwood, M.J., Suarez, D.L., Spackman, E., and Swayne, D.E. 2007. Age at infection affects the pathogenicity of Asian highly pathogenic avian influenza H5N1 viruses in ducks. Virus Res. July 19; [Epub ahead of print].

Pasick, J., Handel, K., and Robinson, J. 2005. Intersegmental recombination between the haemagglutinin and matrix genes was responsible for the emergence of a highly pathogenic H7N3 avian influenza virus in British Columbia. J. Gen. Virol. 86, 727-731.

Pattison, I.H. 1965. Resistance of the scrapie agent to formalin. J. Comp. Pathol. 75, 159-164.

Pattyn, S.R., ed. 1978. Ebola Virus Haemorrhagic Fever. Amsterdam, The Netherlands: Elsevier North Holland Biomedical Press.

Pattyn, S.R. 2000. Monkeypox virus infections. Rev. Sci. Tech. Off. Int. Epiz. 19, 92-97. 
Patz, J.A., Daszak, P., Tabor, G.M., Aguirre, A.A., Pearl, M., Epstein, J.,Wolfe, N.D., Kilpatrick, A.M., Foufopoulos, J., Molyneux, D., and Bradley, D.J. 2004.Unhealthy landscapes: Policy recommendations on land use change and infectious disease emergence. Environ. Health Perspect. 112, 1092-1098.

Patz, J.A., and Wolfe, N.D. 2002. Global ecological change and human health. In Conservation medicine: Ecological health in practice, eds. Aguirre, A.A., Ostfeld, R.S., Tabor, G.M., House, C., and Pearl, M.C., 167-181. New York, NY: Oxford University Press.

Pearce-Duvet, J.M. 2006. The origin of human pathogens: Evaluating the role of agriculture and domestic animals in the evolution of human disease. Biol. Rev. Camb. Philos. Soc. 81, 369-382.

Pearson, J., Salman, M.D., BenJabara, K., Brown, C., Formenty, P., Griot, C., James, A., Jemmi, T., King, L., Lautner, E., McCluskey, B.J., Meslin, F.X., and Ragan, V. 2005. Global risks of infectious animal diseases. Council for Agricultural Science and Technology, Issue Paper No. 28.

Pedersden, K.A., Sadasiv, E.C., Chang, P.W., and Yates, V.J. 1990. Detection of antibody to avian viruses in human populations. Epidemiol. Infect. 10 4, 519-525.

Peeters, M. 2004. Cross-species transmissions of simian retroviruses in Africa and risk for human health. Lancet 363, 911-912.

Peeters, M., Courgnaud, V., Abela, B., Auzel, P., Pourrut, X., Bibollet-Ruche, F., Loul, S., Liegeois, F., Butel, C., Koulagna, D., Mpoudi-Ngole, E., Shaw, G.M., Hahn, B.H., and Delaporte, E. 2002. Risk to human health from a plethora of simian immunodeficiency viruses in primate bushmeat. Emerg. Infect. Dis. 8, 451-457.

Peiris, J.S., de Jong, M.D., and Guan, Y. 2007. Avian influenza virus (H5N1): A threat to human health. Clin. Microbiol. Rev. 20, 243-267.

Peiris, J.S., Guan, Y., Markwell, D., Ghose, P., Webster, R.G., and Shortridge, K.F. 2001. Cocirculation of avian H9N2 and contemporary "human" H3N2 influenza A viruses in pigs in southeastern China: Potential for genetic reassortment? J. Virol. 75, 9679-9686.

Peiris, J.S.M., Lai, S.T., Poon, L.L.M., Guan,Y.,Yam, L.Y.C., Lim,W., Nicholls, J.,Yee,W.K.S.,Yan,W.W., Cheung, M.T., Cheng,V.C.C., Chan, K.H., Tsang, D.N.C., Yung, R.W.H., Ng, T.K., and Yuen, K.Y. 2003. Coronavirus as a possible cause of severe acute respiratory syndrome. Lancet 361 , 1319-1325.

Peiris, M., Yuen, K.Y., Leung, C.W., Chan, K.H., Ip, P.L., Lai, R.W., Orr,W.K., and Shortridge, K.F. 1999. Human infection with influenza H9N2. Lancet 354, 916-917.

Pennington, J.H., Brooksbank, N.H., Pool, P.M., and Seymour, F. 1968. SalmonellaVirchowin a chickenpacking station and associated rearing units. Br. Med. J. 4, 804-806.

Perdue, M.L., Crawford, J.M., Garcia, M., Latimer, J., and Swayne, D.E. 1997. Occurrence and possible mechanisms of cleavage site insertions in the avian influenza hemagglutinin gene. In Proceedings of the 4th International Symposium on Avian Influenza. U.S. Animal Health Association, Athens, Georgia, 182-193.

Perdue, M.L., and Swayne, D.E. 2005. Public health risk from avian influenza viruses. Avian Dis. 49, 317-327.

Perez, D.R.,Webby, R.J., andWebster, R.G. 2003. Land-based birds as potential disseminators of avian/mammalian reassortant influenzaAviruses. Avian Dis. 47, 1114-1117.

Perkins, L.E., and Swayne, D.E. 2002. Pathogenicity of a Hong Kong-origin H5N1 highly pathogenic avian influenza virus for emus, geese, ducks, and pigeons. Avian Dis. 46, 53-63.

Perroncito, E. 1878. Epizoozia tifoide nei gallinacei. Ann. Acad. Agric. 21, 87.

Perry, R.D., and Fetherston, J.D. 1997.Yersinia pestis: Etiologic agent of plague. Clin. Microbiol. Rev. 10, 35-66.

Peterson, M.C. 1994. Clinical aspects of Campylobacter jejuni infections in adults. West J. Med. 161, 148-152.

Phua, K., and Lee, L.K. 2005. Meeting the challenges of epidemic infectious disease outbreaks: An agenda for research. J. Public Health Policy 26, 122-132.

Pimm, S.L., Ayres, M., Balmford, A., Branch, G., Brandon, K., Brooks, T., R. Bustamante, Costanza, R., Cowling, R., Curran, L.M., Dobson, A., Farber, S., da Fonseca, G.A., Gascon, C., Kitching, R., McNeely, J., Lovejoy, T., Mittermeier, R.A., Myers, N., Patz, J.A., Raffle, B., Rapport, D., Raven, P., Roberts, C., Rodriguez, J.P., Rylands, A.B., Tucker, C., Safina, C., Samper, C., Stiassny, M.L., Supriatna, J., Wall, D.H., and Wilcove, D. 2001. Can we defy nature's end? Science 293, 2207-2208. 
Pond, J., and Pond, W. 2000. Introduction to Animal Science. New York, NY: John Wiley \& Sons, Inc. Potter, C.W. 2001. A history of influenza. J. Appl. Microbiol. 91, 572-579.

Powell, D.G., Watkins, K.L., Li, P.H., and Shortridge, K.F. 1995. Outbreak of equine influenza among horses in Hong Kong during 1992. Vet. Rec. 136, 531.

Power, C. 2005. The source and means of spread of the avian influenza virus in the Lower Fraser Valley of British Columbia during an outbreak in the winter of 2004: An interim report. Canadian Food Inspection Agency.

Price, L.B., Johnson, E., Vailes, R., and Silbergeld, E. 2005. Fluoroquinolone-resistant Campylobacter isolates from conventional and antibiotic-free chicken products. Environ. Health Perspect. 113, 557-560.

Proctor, N.S., and Lynch, P.J. 1993. Manual of Ornithology: Avian Structure and Function. New Haven, CT: Yale University Press.

Prusiner, S.B. 1998. Prions. Proc. Natl. Acad. Sci. USA 95, 13363-13383.

Purdue Research Foundation. 2001. Beef and dairy cows: Livestock manure handling on the farm.

Puvadolpirod, S., and Thaxton, J.P. 2000.Model of physiological stress in chickens 1. Response parameters. Poult. Sci. 79, 363-369.

Puzelli, S., Di Trani, L., Fabiani, C., Campitelli, L., De Marco, M.A., Capua, I., Aguilera, J.F., Zambon, M., and Donatelli, I. 2005. Serological analysis of serum samples from humans exposed to avian $\mathrm{H} 7$ influenza viruses in Italy between 1999 and 2003. J. Infect. Dis. 192, 1318-1322.

Pyle, G.F. 1986. The Diffusion of Influenza: Patterns and Paradigms. Totowa, NJ: Rowman \& Littlefield.

Pyykko, I., Vesanen, M., Asikainen, K., Koskiniemi, M., Airaksinen, L., and Vaheri, A. 1994. Human spumaretrovirus in the etiology of sudden hearing loss. Acta Otolaryngol. (Stockh.) 114, 224.

Qu, X.X., Hao, P., Song, X.J., Jiang, S.M., Liu, Y.X.,Wang, P.G., Rao, X., Song, H.D.,Wang, S.Y., Zuo,Y., Zheng, A.H., Luo, M.Wang, H.L., Deng, F.,Wang, H.Z., Z.H. Hu, Ding, M.X., Zhao, G.P., and Deng, H.K. 2005. Identification of two critical amino acid residues of the severe acute respiratory syndrome coronavirus spike protein for its variation in zoonotic tropism transition via a double substitution strategy. J. Biol. Chem. 280, 29588-29595.

Rankins, Jr., D.L., Poore, M.H., Capucille, D.J., and Rogers, G.M. 2002. Recycled poultry bedding as cattle feed. Vet. Clin. North Am. Food Anim. Pract. 18, 253-266.

Rappole, J.H., Derrickson, S.R., and Hubalek, Z. 2000. Migratory birds and spread of West Nile virus in the western hemisphere. Emerg. Infect. Dis. 6, 319-328.

Rauw, W.M., Kanis, E., E.N. Noordhuizen-Stassen, and Grommers, F.J. 1998. Undesirable side effects of selection for high production efficiency in farm animals: A review. Livest. Prod. Sci. 56, 15-33.

Ravenholt, R.T., and Foege,W.H. 1982. 1918 Influenza, encephalitis lethargica, Parkinsonism. Lancet $320,860-864$.

Redrobe, S.P. 2007. Avian influenza H5N1: A review of the current situation and relevance to zoos. Int. Zoo Yearb. 41, 96-109.

Reid, A.H., McCall, S., Henry, J.M., and Taubenberger, J.K. 2001a. Experimenting on the past: The enigma of von Economo's encephalitis lethargica. Neuropathol, J. Exp. Neurol. 60, 663-670.

Reid, A.H., Taubenberger, J.K., and Fanning, T.G. 2001b. The 1918 Spanish influenza: Integrating history and biology. Microb. Infect. 3, 81-87.

Reiter, P., and Sprenger, D. 1987. The used tire trade: A mechanism for the worldwide dispersal of container-breeding mosquitoes. J. Am. Mosq. Control Assoc. 3, 494-501.

Rhodes, G., Huys, G., Swings, J., McGann, P., Hiney, M., Smith, P., and R.W. Pickup. 2000. Distribution of oxytetracycline resistance plasmids between aeromonads in hospital and aquaculture environments: Implication of Tn1721 in dissemination of the tetracycline resistance determinant tet A. Appl. Environ. Microbiol. 66, 3883-3890.

Richmond, M. 1990. The microbial safety of foods, parts I \& II. Report of the Committee on the Microbial Safety of Foods. London: Her Majesty's Stationery Office.

Rigoni, M., Shinya, K., Toffan, A., Milani, A., Bettini, F., Kawaoka, Y., G. Cattoli, and Capua, I. 2007. Pneumo- and neurotropism of avian origin Italian highly pathogenic avian influenza H7N1 isolates in experimentally infected mice. Virology 364, 28-35.

Riley, L.W., Remis, R.S., Helgerson, S.D., Mcgee, H.B., Wells, J.G., Davis, B.R., Hebert, R.J., Olcott, E.S., Johnson, L.M., Hargrett, N.T., Blake, P.A., and Cohen, M.L. 1983. Hemorrhagic colitis associated with a rare Escherichia-Coli serotype. N. Engl. J. Med. 30 8, 681-685.

Riley, P.Y., and Chomel, B.B. 2005. Hedgehog zoonoses. Emerg. Infect. Dis. 11, 1-5. 
Rimmelzwaan, G.F., Kuiken, T., van Amerongen, G., Bestebroer, T.M., Fouchier, R.A., and Osterhaus, A.D. 2001. Pathogenesis of influenza A (H5N1) virus infection in a primate model. J. Virol. 75, 6687-6691.

Rimmelzwaan, G.F., Kuiken, T., van Amerongen, G., Bestebroer, T.M., Fouchier, R.A., and Osterhaus, A.D. 2003. A primate model to study the pathogenesis of influenza $A(H 5 N 1)$ virus infection. Avian Dis. 47, 931-933.

Ritchie, B.W. 1995. Avian Viruses: Function and Control. Lake Worth, FL: Wingers Publishing.

Ritvo, H. 2004. Animal planet. Envir. Hist. 9, 204.

Robbins, K.E., Lemey, P., Pybus, O.G., Jaffe, H.W., Youngpairoj, A.S., Brown, T.M., Salemi, M., Vandamme, A.M., and Kalish, M.L. 2003. U.S. Human immunodeficiency virus type 1 epidemic: Date of origin, population history, and characterization of early strains. J. Virol. 77, 6359-6366.

Roberton, S.I., Bell, D.J., Smith, G.J., Nicholls, J.M., Chan, K.H., Nguyen, D.T., Tran, P.Q., Streicher, U., Poon, L.L., Chen, H., Horby, P., Guardo, M., Guan, Y., and Peiris, J.S. 2006. Avian influenza H5N1 in viverrids: Implications for wildlife health and conservation. Proc. Biol. Sci. 273, 1729 1732.

Roberton, S., Trung, T.C., and Momberg, F. 2003. Hunting and trading wildlife: An investigation into thewildlife trade in and around the PuMatNational Park, Nghe An Province, Vietnam. Nghe An, Vietnam: SFNC Project Management Unit.

Robinson, C.M., Sinclair, J.F., Smith,M.J., and O'Brien, A.D. 2006. Shiga toxin of enterohemorrhagic Escherichia coli type 0157:H7 promotes intestinal colonization. Proc. Natl. Acad. Sci. USA 10 3, 9667-9672.

Rocourt, J. 1996. Risk factors for listeriosis. Food Control 7, 195-202.

Rodrigo, M.J., and Dopazo, J. 1995. Evolutionary analysis of the Picornavirus family. J. Mol. Evol. 40, 362-371.

Rodriguez-Siek, K.E., Giddings, C.W., Doetkott, C., Johnson, T.J., Fakhr, M.K., and Nolan, L.K. 2005. Comparison of Escherichia coli isolates implicated in human urinary tract infection and avian colibacillosis. Microbiology 151, 2097-2110.

Rogers, G.N., and Paulson, J.C. 1983. Receptor determinants of human and animal influenza virus isolates: Differences in receptor specificity of the $\mathrm{H} 3$ hemagglutinin based on species of origin. Virology 127, 361-373.

Rohm, C., Horimoto, T., Kawaoka, Y., Suss, J., and Webster, R.G. 1995. Do hemagglutinin genes of highly pathogenic avian influenza viruses constitute unique phylogenetic lineages? Virology 209 , 664-670.

Rojas, H., Moreira, R., Avalos, P., Capua, I., and Marangon, S. 2002. Avian influenza in poultry in Chile. Vet. Rec. 151, 188.

Rosenau, M.J., and Last, J.M. 1980. Maxcy-Rosenau Preventative Medicine and Public Health. New York, NY: Appleton-Century-Crofts.

Rosenquist, H., Nielsen, N.L., Sommer, H.M., Norrung, B., Van Pelt,W.,W.J.B. Wannet, van de Giessen, A.W., Mevius, D.J., and van Duynhoven, Y.T.H.P. 2004. Trends in gastroenteritis (GE) in the Netherlands, 1996-2003. Infectieziekten Bull. 15, 335-341.

Roth, J.A., and Flaming, K.P. 1990. Model systems to study immunomodulation in domestic food animals. Adv. Vet. Sci. Comp. Med. 35, 21-41.

Rott, R. 1992. The pathogenic determinant of influenza virus. Vet. Microbiol. 33, 303-310.

Roucoux,D.F., andMurphy, E.L. 2004. The epidemiology and disease outcomes of human Tlymphotropic virus type II. AIDS Rev. 6, 144-154.

Rowe, T., Cho, D.S., Bright, R.A., Zitzow, L.A., and Katz, J.M. 2003. Neurological manifestations of avian influenza viruses in mammals. Avian Dis. 47, 1122-1126.

Rude, B.J., and Rankins, D.L., Jr. 1997. Mineral status in beef cows fed broiler litter diets with cationanion differences or supplemented with hay. J. Anim. Sci. 75, 727-735.

Russell, S.M., and Fairchild, B.D. 2005. Poultry production China's way. Watt Poultry USA 6, 26-30.

Russo, T.A., and Johnson, J.R. 2003. Medical and economic impact of extraintestinal infections due to Escherichia coli: An overlooked epidemic. Microb. Infect. 5, 449-456.

Rybicki, E.P., and Pietersen, G. 1999. Plant virus disease problems in the developing world. Adv. Virus Res. 53, 127-175.

Sabirovic, M. 2004. Qualitative risk analysis: HPAl in ostriches in South Africa. United Kingdom Department of Environment, Food and Rural Affairs. 
Salman, M.D., and Meyer, M.E. 1984. Epidemiology of bovine brucellosis in the Mexicali Valley, Mexico: Literature review of disease-associated factors. Am. J. Vet. Res. 45, 1557-1560.

Samuel, M.C., Vugia, D.J., Shallow, S., Marcus, R., Segler, S., McGivern, T., Kassenborg, H., Reilly, K., Kennedy, M., Angulo, F., Tauxe, R.V., and Emerging Infections Program FoodNet Working Group. 2004. Epidemiology of sporadic Campylobacter infection in the United States and declining trend in incidence, FoodNet 1996-1999. Clin. Infect. Dis. 38, S165-S174.

Sanderson, M.W., Gay, J.M., and Baszler, T.V. 2000. Neospora caninum seroprevalence and associated risk factors in beef cattle in the northwestern United States. Vet. Parasitol. 90, 15-24.

Sanford, S.E., and Higgins, R. 1992. Streptococcal diseases. In Diseases of swine, ed. Leman, A.D., 588-598. Ames, lowa: lowa State University Press.

Sano, T. 1998. Control of fish disease, and the use of drugs and vaccines in Japan. J. Appl. Ichthyol. 14, 131-137.

Sapkota, A.R., Lefferts, L.Y., McKenzie, S., and Walker, P. 2007. What do we feed to food-production animals? A review of animal feed ingredients and their potential impacts on human health. Environ. Health Perspect. 115, 663-670.

Schall, J.J. 2002. Parasite virulence. In The Behavioral Ecology of Parasites, eds. Lewis, E.E., Campbell, J.F., and Sukhdeo, M.V.K., 283-313. Wallingford, UK: CAB International.

Schat, K.A., and Davies, C.J. 2000. Viral diseases. In Breeding for disease resistance in farm animals, eds. Axford, R.F.E., Bishop, S.C., Nicholas, F.W., and Owen, J.B., 271-300. Wallingford, UK: $\mathrm{CAB}$ International.

Schillaci, M.A., Jones-Engel, L., Engel, G.A., Paramastri, Y., Iskandar, E.,Wilson, B., Allan, J.S., Kyes, R.C.,Watanabe, R., and Grant, R. 2005. Prevalence of enzootic simian viruses among urban performance monkeys in Indonesia. Trop. Med. Int. Health 10, 1305-1314.

Schneider, M.C., Belotto, A., Ade, M.P., Leanes, L.F., Correa, E., Tamayo, H., Medina, G., and Rodrigues, M.J. 2005. Epidemiologic situation of human rabies in Latin America in 2004. Epidemiol. Bull. 26, 2-4.

Scholtissek, C. 1990. Pigs as the 'mixing vessel' for the creation of new pandemic influenza A viruses. Med. Princ. Pract. 2, 65-71.

Scholtissek, C., and Naylor, E. 1998. Fish farming and influenza pandemics. Nature 331, 215.

Scholtissek, C., Rohde,W., Von-Hoyningen, V., and Rott, R. 1978. On the origin of the human influenza virus subtypes H2N2 and H3N2. Virology 87, 13-20.

Schrag, S., and Wiener, P. 1995. Emerging infectious disease: What are the relative roles of ecology and evolution? Trends Ecol. Evolut. 10, 319-324.

Schuchat, A., Swaminathan, B., and Broome, C.V. 1991. Epidemiology of human listeriosis. Clin. Microbiol. Rev. 4, 169-183.

Schweizer, M., Falcone, V., Gange, J., Turek, R., Neumann-Haefelin, D. 1997. Simian foamy virus isolated from an accidentally infected human individual. J. Virol. 71, 4821-4824.

Schweizer, M., and Neumann-Haefelin, D. 1995. Phylogenetic analysis of primate foamy viruses by comparison of pol sequences. Virology $207,577-582$.

Schweizer, M., Turek, R., Hahn, H., Schliephake, A., Netzer, K.O., Eder, G., Reinhardt, M., Rethwilm, A., and D. Neumann-Haefelin. 1995. Markers of foamy virus infections in monkeys, apes, and accidentally infected humans: Appropriate testing fails to confirm suspected foamy virus prevalence in humans. AIDS Res. Hum. Retroviruses. 11, 161-170.

Scott, G.R. 1983. The History of Cockfighting. Hindhead: Triplegate.

Seal, B.S., King, D.J., and Sellers, H.S. 2000. The avian response to Newcastle disease virus. Dev. Comp. Immunol. 24, 257-268.

Selgelid, M.J. 2005. Ethics and infectious disease. Bioethics 19, 272-289.

Selvey, L., Taylor, R., Arklay, A., and Gerrard, J. 1996. Screening of bat carers for antibodies to equine morbillivirus. Commun. Dis. Intell. 20, 477-478.

Senne, D.A., Pederson, J.C., and Panigrahy, B. 2003. Live-bird markets in the northeastern United States: A source of avian influenza in commercial poultry. In Proceedings of the Frontis Workshop on Avian Influenza: Prevention and Control, eds. Schrijver, R.S. and Koch, G., 19-24. Wageningen, The Netherlands.

Serratosa, J., Ribo, O., Correia, S., and Pittman, M. 2007. EFSA scientific risk assessment on animal health and welfare aspects of avian influenza (EFSA-Q-2004-075). Avian Dis. 51, 501-503. 
Shane, S.M. 2000. Campylobacter infection of commercial poultry. Rev. Sci. Tech. Off. Int. Epiz. 19, 376395.

Shane, S.M. 2003. Disease continues to impact the world's poultry industries. World Poultry 19, 22-27.

Sharp, G.B., Kawaoka, Y., Wright, S.M., Turner, B., Hinshaw, V., and Webster, R.G. 1993.Wild ducks are the reservoir for only a limited number of influenza A subtypes. Epidemiol. Infect. 110, 161-176.

Sharp, P.M., Bailes, E., Chaudhuri, R.R., Rodenburg, C.M., Santiago, M.O., and Hahn, B.H. 2001. The origins of acquired immune deficiency syndrome viruses: Where and when? Philos. Trans. $R$. Soc. Lond. B. Biol. Sci. 356, 867-876.

Sheng, H., Lim, J.Y., Knecht, H.J., Li, J., and Hovde, C.J. 2006. Role of Escherichia coli O157:H7 virulence factors in colonization at the bovine terminal rectal mucosa. Infect. Immun. 74, 46854693.

Shields, D.A., and Matthews, K.H., Jr. 2003. Interstate Livestock Movements, LPD-M-108-01. U.S. Department of Agriculture Economic Research Service.

Shinya, K., Ebina, M., Yamada, S., Ono, M., Kasai, N., and Kawaoka, Y. 2006. Avian flu: Influenza virus receptors in the human airway. Nature 440, 435-436.

Shortridge, K.F. 1982. Avian influenza viruses of southern China and Hong Kong: Ecological aspects and implications for man. Bull.World Health Organ. 60, 129-135.

Shortridge, K.F. 1992. Pandemic influenza: A zoonosis? Sem. Respir. Infect. 7, 11-25.

Shortridge, K.F. 1999. Poultry and the influenza H5N1 outbreak in Hong Kong, 1997, Abridged chronology and virus isolation. Vaccine 17, S26-S29.

Shortridge, K.F. 2003a. Avian influenza viruses in Hong Kong: Zoonotic considerations. In Proceedings of the FrontisWorkshop on Avian Influenza: Prevention and Control, eds. Schrijver, R.S. and Koch, G., 9-18. Wageningen, The Netherlands.

Shortridge, K.F. 2003b. Severe acute respiratory syndrome and influenza. Am. Resp, J. Crit. Care Med. 168, 1416-1420.

Shortridge, K.F., Gao, P., Guan, Y., Ito, T., Kawaoka, Y., Markwell, D., Takada, A., and Webster, R.G. 2000. Interspecies transmission of influenza viruses: H5N1 virus and a Hong Kong SAR perspective. Vet. Microbiol. 74, 141-147.

Shortridge, K.F., Peiris, J.S., and Guan, Y. 2003. The next influenza pandemic: Lessons from Hong Kong. J. Appl. Microbiol. 94, 70S-79S.

Shortridge, K.F., and Stuart-Harris, C.H. 1982. An influenza epicentre? Lancet 2, 212-213.

Shortridge, K.F., Zhou, N.N., Guan, Y., Gao, P., Ito, T., Kawaoka, Y., Kodihalli, S., Krauss, S., Markwell, D., Murti, K.G., Norwood, M., Senne, D., Sims, L., Takada, A., and Webster, R.G. 1998. Characterization of avian H5N1 influenza viruses from poultry in Hong Kong. Virology 252, 331342.

Siddell, S. 1995. The Coronaviridae. New York, NY: Plenum Press. Siegel, H.S. 1983. Effects of intensive production methods on livestock health. Agro-Ecosystems 8, 215-230.

Silbergeld, E. 2006. Avian influenza risks and the animal-human interface. In Avian Flu: The Pandemic Threat and the Global Strategy at the Johns Hopkins Bloomberg School of Public Health, January 30. <commprojects.jhsph.edu/ media/009 avian flu.ram>.

Simpson, J.R., Shi, Y., Li, O., Chen,W., and Liu, S. 1999. Pig, broiler and laying hen farm structure in China, 1996. Proposal to International Agrohydrology Research and Training Center (IARTC) International Symposium, June 25-26.

Sims, L.D., Domenech, J., Benigno, C., Kahn, S., Kamata, A., Lubroth, J., Martin, V., and Roeder, P. 2005. Origin and evolution of highly pathogenic H5N1 avian influenza in Asia. Vet. Rec. 157, 159-164.

Sims, L.D., Ellis, T.M., Liu, K.K., Dyrting, K., Wong, H., Peiris, M., Guan, Y., and Shortridge, K.F. 2003. Avian influenza in Hong Kong 1997-2002. Avian Dis. 47, 832-838.

Sinclair, M.C., Nielsen, B.L., Oldham, J.D., and Reid,H.W. 1999. Consequences for immune function of metabolic adaptations to load. In Metabolic stress in dairy cows, eds. Oldham, J.D., Simm, G., Growen, A.F., Nielsen, B.L., Pryce, J.F., and Lawrence, T.L.J., 113-118. Edinburgh, UK: British Society of Animal Science.

Skyberg, J.A., Johnson, T.J., Johnson, J.R., Clabots, C., Logue, C.M., and L.K. Nolan. 2006. Acquisition of avian pathogenic Escherichia coli plasmids by a commensal $\mathrm{E}$. coli isolate enhances its abilities to kill chicken embryos, grow in human urine, and colonize the murine kidney. Infect. Immun. 74, 6287-6292. 
Slingenbergh, J.I., Gilbert, M., de Balogh, K.I., and Wint, W. 2004. Ecological sources of zoonotic diseases. Rev. Sci. Tech. Off. Int. Epiz. 23, 467-484.

Smith, D.L., Dushoff, J., and Morris, J.G. 2005. Agricultural antibiotics and human health. PLoS Med. 2, e232.

Smith, G.L., Irving, W.L., McCauley, J.W., and D.J. Rowlands (eds.). 2001. New Challenges to Health: The Threat of Virus Infection. Cambridge, UK: Cambridge University Press.

Smith, G.J., Naipospos, T.S., Nguyen, T.D., de Jong, M.D., Vijaykrishna, D., Usman, T.B., Hassan, S.S., Nguyen, T.V., Dao, T.V., Bui, N.A., Leung, Y.H., C.L. Cheung, Rayner, J.M., Zhang, J.X., Zhang, L.J., Poon, L.L., Li, K.S., Nguyen, V.C., T.T. Hien, Farrar, J., Webster, R.G., Chen, H., Peiris, J.S., and Guan, Y. 2006. Evolution and adaptation of H5N1 influenza virus in avian and human hosts in Indonesia and Vietnam. Virology 350, 258-268.

Smith, J.L., Fratamico, P.M., and Gunther, N.W. 2007. Extraintestinal pathogenic Escherichia coli. Foodborne Pathog. Dis. 4, 134-163.

Smith, T. 1934. Parasitism and Disease. Princeton, NJ: Princeton University Press.

Smolinksi, M.S., Hamburg, M.A., and Lederberg, J., eds. 2003. Microbial Threats to Health: Emergence, Detection and Response. Washington, D.C.: National Academies Press.

Song, H.D., Tu, C.C., Zhang, G.W., Wang, S.Y., Zheng, K., Lei, L.C., Chen, Q.X., Gao, Y.W., Zhou, H.Q., Xiang, H., Zheng, H.J., Chern, S.W., Cheng, F., Pan, C.M., Xuan, H., Chen, S.J., Luo, H.M., Zhou, D.H., Liu, Y.F., He, J.F., Qin, P.Z., Li, L.H., Ren, Y.Q., Liang, W.J., Yu, Y.D., Anderson, L., Wang, M., Xu, R.H., Wu, X.W., Zheng, H.Y., Chen, J.D., Liang, G., Gao, Y., Liao, M., Fang, L., Jiang, L.Y., Li, H., Chen, F., Di, B., He, L.J., Lin, J.Y., Tong, S., Kong,X.,Du,L.,Hao, P.,Tang,H.,Bernini,A.,Yu,X.J., Spiga,O.,Guo,Z.M., Pan, H.Y., He, W.Z., Manuguerra, J.C., Fontanet, A., Danchin, A., Niccolai, N., Li, Y.X., Wu, C.I., and Zhao, G.P. 2005. Cross-host evolution of severe acute respiratory syndrome coronavirus in palm civet and human. Proc. Natl. Acad. Sci. USA 10 2, 2430-2435.

Songserm, T., Jam-On, R., Sae-Heng, N., and Meemak, N. 2006. Survival and stability of HPAI H5N1 in different environments and susceptibility to disinfectants. In Proceedings of the OIE/FAO International Scientific Conference on Avian Influenza, eds. Schudel, A. and Lombard, M., Vol. 124, 254. Karger, Switzerland: International Association for Biologicals.

Spala, G., Panagiotopoulos, T., Mavroidi, N., Dedoukou, X., Baka, A., Tsonou, P., Triantafyllou, P., Mentis, A.,Kyriazopoulou,V., Melidou, A., and Tsiodras, S. 2006. A pseudo-outbreak of human A/H5N1 infections in Greece and its public health implications. Euro Surveill. 11, 263-267.

Specter, M. 2005. Nature's bioterrorist. New Yorker, February 28, 52-61.

Spika, J.S.,Waterman, S.H., Hoo,G.W., St Louis, M.E., Pacer, R.E., James, S.M., Bissett, M.L., Mayer, L.W., Chiu, J.Y., and Hall, B. 1987. Chloramphenicol-resistant Salmonella newport traced through hamburger to dairy farms: A major persisting source of human salmonellosis in California. $N$. Engl. J. Med. 316, 565-570.

Sreevatsan, S., Pan, X., Stockbauer, K.E., Connell, N.D., Kreiswirth, B.N., Whittam, T.S., and Musser, J.M. 1997. Restricted structural gene polymorphism in the Mycobacterium tuberculosis complex indicates evolutionarily recent global dissemination. Proc. Natl. Acad. Sci. USA 94, 9869-9874.

Srikosamatara, S., Siripholdej, B., and Suteethorn, V. 1992. Wildlife trade in Lao PDR and between Lao PDR and Thailand. Nat. Hist. Bull. Siam. Soc. 40, 1-47.

Sriskandan, S., and Slater, J.D. 2006. Invasive disease and toxic shock due to zoonotic Streptococcus suis: An emerging infection in the East? PLoS Med. 3, e187.

Staats, J.J., Feder, I., Okwumabua, O., and Chengappa,M.M. 1997. Streptococcus suis: Past and present. Vet. Res. Commun. 21, 381-407.

Stacey, K.F., Parsons, D.J., Christiansen, K.H., and Burton, C.H. 2007. Assessing the effect of interventions on the risk of cattle and sheep carrying Escherichia coli $\mathrm{O} 157: \mathrm{H} 7$ to the abattoir using a stochastic model. Prev. Vet. Med. 79, 32-45.

Stamm,W.E., and Norrby, S.R. 2001. Urinary tract infections: Disease panorama and challenges. J. Infect. Dis. 183, S1-S4.

Stanley, J., Linton, D., Burnens, A.P., Dewhirst, F.E., On, S.L., Porter, A., Owen, R.J., and Costas, M. 1994. Helicobacter pullorum sp. nov.-genotype and phenotype of a new species isolated from poultry and from human patients with gastroenteritis. Microbiology 140, 3441-3449. 
Stegeman, A. 2003. Workshop 1, Introduction and spread of avian influenza. In Proceedings of the Frontis Workshop on Avian Influenza: Prevention and Control, eds. Schrijver, R.S. and Koch, G. $<$ library.wur.nl/frontis/avian influenza/workshop1.pdf>.

Steinfeld, H., Gerber, P.,Wassenaar, T., Castel, V., Rosales, M., and de Haan, C. 2006. Livestock's long shadow: Environmental issues and options. Food and Agriculture Organization of the United Nations.

Stevens, J., Blixt, O., Paulson, J.C., and Wilson, I.A. 2006. Glycan microarray technologies: Tools to survey host specificity of influenza viruses. Nat. Rev. Microbiol. 4, 857-864.

Stieneke-Grober, A., Vey, M., Angliker, H., Shaw, E., Thomas, G., Roberts, C., Klenk, H.D., and Garten, W. 1992. Influenza virus hemagglutinin with multibasic cleavage site is activated by furin, a subtilisin-like endoprotease. EMBO J. 11, 2407-2414.

Stiles, G.E. 1968. Serologic screening of rhesus and grivet monkeys for SV40 and the foamy viruses. Proc. Soc. Exp. Biol. Med. 127, 225-230.

Stratton, K., Alamario, D.A., and McCormick, M.C., eds. 2002. Immunization Safety Review: SV40 Contamination of Polio Vaccine and Cancer. Washington, D.C.: National Academies Press.

Sturm-Ramirez, K.M., Ellis, T., Bousfield, B., Bissett, L., Dyrting, K., Rehg, J.E., Poon, L., Guan, Y., Peiris, M., and Webster, R.G. 2004. Reemerging H5N1 influenza viruses in HongKong in 2002 are highly pathogenic to ducks. J. Virol. 78, 4892-4901.

Sturm-Ramirez, K.M., D.J. Hulse-Post, Govorkova, E.A., Humberd, J., Seiler, P., Puthavathana, P., Buranathai, C., Nguyen, T.D., Chaisingh, A., Long, H.T., Naipospos,T.S., Chen, H., Ellis,T.M., Guan,Y., Peiris, J.S., andWebster, R.G. 2005. Are ducks contributing to the endemicity of highly pathogenic H5N1 influenza virus in Asia? J. Virol. 79, 11269-11279.

Stutman, H.R. 1994. Salmonella, Shigella, and Campylobacter: Common bacterial causes of infectious diarrhea. Pediatr. Ann. 23, 538-543.

Su, C., Evans, D., Cole, R.H., Kissinger, J.C., Ajioka, J.W., and Sibley, L.D. 2003. Recent expansion of Toxoplasma through enhanced oral transmission. Science 299, 414-416.

Suarez, D.L. 2000. Evolution of avian influenza viruses. Vet. Microbiol. 74, 15-27.

Suarez, D.L., Garcia, M., Latimer, J., Senne, D., and Perdue, M. 1999. Phylogenetic analysis of H7 avian influenza viruses isolated from the live bird markets of the northeastern United States. J. Virol. 73, 3567-3573.

Suarez, D.L., Senne, D.A., Banks, J., Brown, I.H., Essen, S.C., Lee, C.W., Manvell, R.J., MathieuBenson, C., Moreno, V., Pedersen, J.C., Panigrahy, B., Rojas, H., E. Spackman, and Alexander, D.J. 2004. Recombination resulting in virulence shift in avian influenza outbreak, Chile. Emerg. Infect. Dis. 10, 693-699.

Suarez, D.L., Spackman, E., and Senne, D.A. 2003. Update on molecular epidemiology of H1, H5, and $\mathrm{H} 7$ influenza virus infections in poultry in North America. Avian Dis. 47, 888-897.

Subbarao, K., and Katz, J. 2000. Avian influenza viruses infecting humans. Cell. Mol. Life Sci. 57, 17701784.

Suerbaum, S., and Michetti, P. 2002. Helicobacter pylori infection. N. Eng. J. Med. 347, 1175-1186.

Sulaiman, I.M., Xiao, L.H., Yang, C.F., Escalante, L., Moore, A., Beard, C.B., Arrowood, M.J., and Lal, A.A. 1998. Differentiating human from animal isolates of Cryptosporidium parvum. Emerg. Infect. Dis. 4, 681-685.

Sutherst, R.W. 2004. Global change and human vulnerability to vector-borne diseases. Clin. Microbiol. Rev. 17, 136-173.

Swayne, D.E. 2006. Occupational and consumer risks from avian influenza viruses. Dev. Biol. 124, 8590.

Swayne, D.E., and Beck, J.R. 2004. Heat inactivation of avian influenza and Newcastle disease viruses in egg products. Avian Pathol. 33, 512-518.

Swayne, D.E., and Beck, J.R. 2005. Experimental study to determine if low-pathogenicity and highpathogenicity avian influenza viruses can be present in chicken breast and thigh meat following intranasal virus inoculation. Avian Dis. 49, 81-85.

Swildens, B., Stockhofe-Zurwieden, N., van der Meulen, J., Wisselink, H.J., Nielen, M., and Niewold, T.A. 2004. Intestinal translocation of Streptococcus suis type 2 EF+ in pigs. Vet. Microbiol. 10 3, 2933. 
Switzer, W.M., Bhullar, V., Shanmugam, V., Cong, M.E., Parekh, B., Lerche, N.W., Yee, J.L., Ely, J.J., Boneva, R., Chapman, L.E., Folks, T.M., and Heneine, W. 2004. Frequent simian foamy virus infection in persons occupationally exposed to nonhuman primates. J. Virol. 78, 2780-2789.

Switzer,W., Kalish, M.,Yang, C., Garcia, A., Wright, A., Folks, T., and Heneine, W. 2005. Simian foamy virus infection among HIV-1 positive sex workers and blood donors in central Africa. Proceedings of the 12th Conference on Retroviruses and Opportunistic Infections, February 22-25, Boston. $<$ ww2.aegis.org/conferences/CROI/2005/308.html>.

Synnott, M.B., Morse, D.L., and Hall, S.M. 1994. Neonatal meningitis in England and Wales: A review of routine national data. Arch. Dis. Child. 71, F75-F80.

Takakuwa, H., Ito, T., Takada, A., Okazaki, K., and Kida, H. 1998. Potentially virulent Newcastle disease viruses are maintained in migratory waterfowl populations. Jpn. J. Vet. Res. 45, 207-215.

Tamura, N., and Kira, S. 1995. Human foamy virus and familial Mediterranean fever in Japan. JAMA 274, 1509.

Tang, J.,Wang, C., Feng,Y.,Yang,W., Song, H., Chen, Z.,Yu, H., Pan, X., Zhou, X., Wang, H., Wu, B., Wang, H., Zhao, H., Lin, Y., Yue, J., Wu, Z., He, X., Gao, F., A.H. Khan,Wang, J., Zhao, G.P.,Wang, Y.,Wang, X., Chen, Z., and Gao, G.F. 2006. Streptococcal toxic shock syndrome caused by Streptococcus suis serotype 2. PLoS Med. 3, e151.

Tashiro, M., Ciborowski, P., Klenk, H.D., Pulverer, G., and Rott, R. 1987. Role of Staphylococcus protease in the development of influenza pneumonia. Nature 325, 536-537.

Taubenberger, J.K. 1998. Influenza virus hemagglutinin cleavage into HA1, HA2: No laughing matter. Proc. Natl. Acad. Sci. USA 95, 9713-9715.

Taubenberger, J.K., and Morens, D.M. 2006. 1918 influenza: The mother of all pandemics. Emerg. Infect. Dis. 12, 15-22.

Taubenberger, J.K., Reid, A.H., Krafft, A.E., Bijwaard, K.E., and Fanning, T.G. 1997. Initial genetic characterization of the 1918 "Spanish" influenza virus. Science 275, 1793-1796.

Taubenberger, J.K., Reid, A.H., Lourens, R.M., Wang, R., Guozhong, J., and Fanning, T.G. 2006. Molecular virology: Was the 1918 pandemic caused by a bird flu? Was the 1918 flu avian in origin? (Reply). Nature 440, E9.

Taubenberger, J.K., Reid, A.H., Lourens, R.M.,Wang, R., Jin, G., and Fanning, T.G. 2005. Characterization of the 1918 influenza virus polymerase genes. Nature 437, 889-893.

Tauxe, R.V. 2002. Emerging foodborne pathogens. Int. J. Food Microbiol. 78, 31-41.

Tauxe, R.V. 1991. Salmonella: A postmodern pathogen. J. Food Prot. 54, 563-568.

Taylor, D. 1997. Seeing the forests for more than the trees. Environ. Health Perspect. 105, 1186-1191.

Taylor, L.H., Latham, S.M., andWoolhouse, M.E.J. 2001. Risk factors for human disease emergence. Philos. Trans. R. Soc. Lond. B. Biol. Sci. 356, 983-989.

Taylor, H.R., and Turner, A.J. 1977. A case report of fowl plague keraconjunctivitis. Br. J. Ophthalmol. 61, 86-88.

Tei, S., Kitajima, N., Takahashi, K., and Mishiro, S. 2003. Zoonotic transmission of hepatitis E virus from deer to human beings. Lancet 362, 371-373.

Thamsborg, S.M., Jørgensen, R.J., Waller, P.J., and Nansen, P. 1996. The influence of stocking rate on gastrointestinal nematode infections of sheep over a two-year grazing period. Vet. Parasitol. 67, 207-224.

Thamsborg, S.M., Roepstorff, A., and Larsen, M. 1999. Integrated and biological control of parasites in organic and conventional production systems. Vet. Parasitol. 84, 169-186.

The Humane Society of the United States (HSUS). 2006. An HSUS report: The economic consequences of adopting alternative production practices to genetic selection for rapid growth in poultry.

Thibault, M., and Blaney, S. 2003. The oil industry as an underlying factor in the bushmeat crisis in Central Africa. Conserv. Biol. 17, 1807-1813.

Thiry, E., Zicola, A., Addie, D., Egberink, H., Hartmann, K., Lutz, H., Poulet, H., and Horzinek, M.C. 2007. Highly pathogenic avian influenza H5N1 virus in cats and other carnivores. Vet. Microbiol. 122, 25-31.

Thoen, C., Lobue, P., and de Kantor, I. 2006. The importance of Mycobacterium bovis as a zoonosis. Vet. Microbiol. 112, 339-345.

Thomas, C., and Swayne, D.E. 2007. Thermal inactivation of H5N1 high pathogenicity avian influenza virus in naturally infected chicken meat. J. Food Prot. 70, 674-680.

Thorpe, C.M. 2004. Shiga toxin-producing Escherichia coli infection. Clin. Infect. Dis. 38, 1298-1303. 
Threlfall, E.J. 2000. Epidemic salmonella typhimurium DT 104: A truly international multiresistant clone. Antimicrob, J. Chemother. 46, 7-10.

Tilman, D., Cassman, K.G., Matson, P.A., Naylor, R., and Polasky, S. 2002. Agricultural sustainability and intensive production practices. Nature 418, 671-677.

Tilman, D., Fargione, J., Wolff, B., D'Antonio, C., Dobson, A., Howarth, R., Schindler, D., Schlesinger, W.H., Simberloff, D., and Swackhamer, D. 2001. Forecasting agriculturally driven global environmental change. Science 292, 281-284.

To, K.F., Chan, P.K., Chan, K.F., Lee, W.K., Lam, W.Y., Wong, K.F., Tang, N.L., Tsang, D.N., Sung, R.Y., Buckley, T.A., Tam, J.S., and Cheng, A.F. 2001. Pathology of fatal human infection associated with avian influenza A H5N1 virus. J. Med. Virol. 63, 242-246.

Tollefson, L., P.J. Fedorka-Cray, and Angulo, F.J. 1999. Public health aspects of antibiotic resistance monitoring in the USA. Acta. Vet. Scand. Suppl. 92, 67-75.

Trenberth, K.E., and Hoar, T.J. 1996. The 1990-1995 El Niño-southern oscillation event: Longest on record. Geophys. Res. Lett. 23, 57-60.

Tu, C., Crameri, G., Kong, X., Chen, J., Sun, Y., Yu, M., Xiang, H., Xia, X., S. Liu, Ren, T., Yu, Y., Eaton, B.T., Xuan, H., and Wang, L.F. 2004. Antibodies to SARS coronavirus in civets. Emerg. Infect. Dis. 10, 2244-2248.

Tumpey,T.M., Basler, F., Aguilar, P.V., Zeng, H., Solorzano, A.,Swayne, D.E., Cox, N.J., Katz, J.M., Taubenberger, J.K., Palese, P., and Garcia-Sastre, A. 2005. Characterization of the reconstructed 1918 Spanish influenza pandemic virus. Science 310, 77-80.

Tumpey, T.M., Maines, T.R., Van Hoeven, N., Glaser, L., Solorzano, A., Pappas, C., Cox, N.J., Swayne, D.E., Palese, P., Katz, J.M., and Garcia-Sastre, A. 2007a. A two-amino acid change in the hemagglutinin of the 1918 influenza virus abolishes transmission. Science 315, 655-659.

Tumpey, T.M., Suarez, D.L., Perkins, L.E., Senne, D.A., Lee, J.G., Lee, Y.J., Mo, I.P., Sung, H.W., and Swayne, D.E. 2002. Characterization of a highly pathogenic H5N1 avian influenza A virus isolated from duck meat. J. Virol. 76, 6344-6355.

Tumpey, T.M., Szretter, K.J., Van Hoeven, N., Katz, J.M., Kochs, G., Haller, O., A. Garcia-Sastre, and Staeheli, P. 2007b. TheMx1 gene protectsmice against pandemic 1918 and highly lethal human H5N1 influenza viruses. J. Virol. Jul 25; [Epub ahead of print].

Turnbull, P.C.B. 1979. Food poisoning with special reference to Salmonella: Its epidemiology, pathogenesis and control. Clin. Gastroenterol. 8, 663-714.

Turner, I.M. 1996. Species loss in fragments of tropical rain forest: A review of the evidence. J. Appl. Ecol. 33, 200-209.

Tweed, S.A., Skowronski, D.M., David, S.T., Larder, A., Petric, M., Lees, W., Li, Y., Katz, J., Krajden, M., Tellier, R., Halpert, C., Hirst, M., Astell, C., Lawrence, D., and Mak, A. 2004. Human illness from avian influenza H7N3, British Columbia. Emerg. Infect. Dis. 10, 2196-2199.

Tyson Foods. 2007. All Tyson® brand fresh chicken to be "raised without antibiotics." June 19.

Uiprasertkul, M., Puthavathana, P., Sangsiriwut, K., Pooruk, P., Srisook, K., Peiris, M., Nicholls, J.M., Chokephaibulkit, K., Vanprapar, N., and Auewarakul, P. 2005. Influenza A H5N1 replication sites in humans. Emerg. Infect. Dis. 11, 1036-1041.

United Egg Producers (UEP). 2004. Industry history.

United Egg Producers (UEP). 2006. Animal Husbandry Guidelines for U.S. Egg Laying Flocks. Alpharetta, GA: United Egg Producers.

United Nations Development Programme (UNDP) Global Environment Facility. 2003. Protecting the environment from the impact of the growing industrialization of livestock production in East Asia. Working paper, Thailand.

Uppal, P.K. 2000. Emergence of Nipah virus in Malaysia. Ann. NY Acad. Sci. 916, 354-357.

U.S. Department of Agriculture (USDA). 1998. Salmonella enteritidis risk assessment: Shell eggs and egg products.

U.S. Department of Agriculture (USDA). 2002. Census of Agriculture, Volume 1 Chapter 1, U.S. National Level Data.

U.S. Department of Agriculture (USDA). 2006. An estimate of the prevalence of BSE in the U.S., April 28.

U.S. Department of Agriculture Agricultural Research Service (USDA ARS). 2006. Animal Health National Action Plan. Washington, D.C.: U.S. Department of Agriculture. 
U.S. Department of Agriculture Animal and Plant Health Inspection Service (USDAAPHIS), Veterinary Services. 1993. Dairy herd management practices focusing on preweaned heifers: April 1991July 1992. National Dairy Heifer Evaluation Project.

U.S. Department of Agriculture Animal and Plant Health Inspection Service (USDA APHIS). 2005a. List of USDA-Recognized Animal Health Status of Countries, Areas Regarding Specific Livestock or Poultry Diseases, April 12.

U.S. Department of Agriculture Animal and Plant Health Inspection Service (USDA APHIS), Veterinary Services, Center for Emerging Issues. 2005b. Streptococcus suis outbreak, swine and human, China: Emerging disease notice.

U.S. Department of Agriculture Food Safety and Inspection Service (USDA FSIS). 2002a. Colorado firm recalls beef trim and ground beef products for possible E. coli O157:H7. Recall Release FSISRC-055-2002, July 19.

U.S. Department of Agriculture Food Safety and Inspection Service (USDA FSIS). 2002b. Pennsylvania firm expands recall of turkey and chicken products for possible listeria contamination. Recall Release FSIS-RC-090-2002, October 12.

U.S. Department of Agriculture Food Safety and Inspection Service (USDA FSIS). 2003. Safety of fresh pork. . . from farm to table.

U.S. Department of Agriculture Food Safety Inspection Service (USDA FSIS). 2005. Production and inspection: BSE rules being strictly enforced.

U.S. Food and Drug Administration (FDA). 2001. Risk assessment on the human health impact of fluoroquinolone resistant Campylobacter associated with the consumption of chicken.

U.S. Food and Drug Administration (FDA). 2005. Final decision of the commissioner: Withdrawal of approval of the new animal drug application for enrofloxacin in poultry. Docket No. 2000N-1571.

U.S. Food and Drug Administration, U.S. Department of Agriculture, and Centers for Disease Control and Prevention (FDA/USDA/CDC). 1998. National Antimicrobial Resistance Monitoring System: Enteric Pathogens.

U.S. Government Accountability Office (GAO). 2001. Weaknesses in meat and poultry inspection pilot should be addressed before implementation. Report to the U.S. Senate Committee on Agriculture, Nutrition, and Forestry, December.

U.S. Government Accountability Office (GAO). 2002. Mad Cow Disease: Improvements in the Animal Feed Ban and Other Regulatory Areas Would Strengthen U.S. Prevention Efforts. GAO-02-183. Washington, D.C.: General Accounting Office.

U.S.Government Accountability Office(GAO). 2005. Homeland security: Much is being done to protect agriculture from a terrorist attack, but important challenges remain. Report to Congressional Requesters, 65.

Utterback, W. 1984. Update on avian influenza through February 21, 1984 in Pennsylvania and Virginia. In Proceedings of the 33rd Western Poultry Disease Conference, 4-7.

Uyeki, T.M., Chong, Y.H., Katz, J.M., Lim, W., Ho, Y.Y., Wang, S.S., Tsang, T.H., Au, W.W., Chan, S.C., Rowe, T., Hu-Primmer, J., Bell, J.C., Thompson, W.W., Bridges, C.B., Cox, N.J., Mak, K.H., and Fukuda, K. 2002. Lack of evidence for human-to-human transmission of avian influenza A (H9N2) viruses in Hong Kong, China 1999. Emerg. Infect. Dis. 8, 154-159.

Valcour, J.E., Michel, P., McEwen, S.A., and Wilson, J.B. 2002: Associations between indicators of livestock farming intensity and incidence of human Shiga toxin-producing Escherichia coli infection. Emerg. Infect. Dis. 8, 252-257.

van Baalen, M., and Sabelis, M.W. 1995. The dynamics of multiple infection and the evolution of virulence. Am. Nat. 146, 881-891.

van Blerkom, L.M. 2003. Role of viruses in human evolution. Yearbk. Phys. Anthropol. 46, 14-46.

Van Borm, S., Thomas, I., Hanquet, G., Lambrecht, B., Boschmans, M., Dupont, G., Decaestecker, M., Snacken, R., and van den Berg, T. 2005. Highly pathogenic H5N1 influenza virus in smuggled Thai eagles, Belgium. Emerg. Infect. Dis. 11, 702-705.

van Boven, M., Koopmans, M., M. Du Ry van Beest Holle, Meijer, A., Klinkenberg, D., Donnelly, C.A., and Heesterbeek, H.J. 2007. Detecting emerging transmissibility of avian influenza virus in human households. PLoS Comput. Biol. 3, e145.

Van der Sluis, W. 2005. Housing conditions affect broiler welfare more than stocking density. World Poultry 21, 22-23. 
Van Heuverswyn, F., and Peeters, M. 2007. The origins of HIV and implications for the global epidemic. Curr. Infect. Dis. Rep. 9, 338-346.

Vandekerchove, D., De Herdt, P., Laevens, H., and Pasmans, F. 2004. Risk factors associated with colibacillosis outbreaks in caged layer flocks. Avian Pathol. 33, 337-342.

Varma, M.G.R. 2001. Kyasanur forest disease. In The encyclopedia of arthropod-transmitted infections, ed. Service, M.W., 254-260. New York, NY: CAB International.

Vazquez-Boland, J.A., Kuhn, M., Berche, P., Chakraborty, T., G. Dominguez-Bernal, Goebel,W., B. Gonzalez-Zorn,Wehland, J., and Kreft, J. 2001. Listeria pathogenesis and molecular virulence determinants. Clin. Microbiol. Rev. 14, 584-640.

Vellinga, A., and Van Loock, F. 2002. The dioxin crisis as experiment to determine poultry-related Campylobacter enteritis. Emerg. Infect. Dis. 8, 19-22.

Vijaykrishna, D., Smith, G.J., Zhang, J.X., Peiris, J.S., Chen, H., and Guan, Y. 2007. Evolutionary insights into the ecology of coronaviruses. J. Virol. 81, 4012-4020.

Vijgen, L., Keyaerts, E., Moes, E., Thoelen, I., Wollants, E., Lemey, P., A.M. Vandamme, and Van Ranst, M. 2005. Complete genomic sequence of human coronavirus OC43: Molecular clock analysis suggests a relatively recent zoonotic coronavirus transmission event. J. Virol. 79, 1595-1604.

Viola, C., and DeVincent, S.J. 2006. Overview of issues pertaining to the manufacture, distribution, and use of antimicrobials in animals and other information relevant to animal antimicrobial use data collection in the United States. Prev. Vet. Med. 73, 111-131.

Vittor, A.Y., Gilman, R.H., Tielsch, J., Glass, G., Shields, T., Lozano, W.S., Pinedo-Cancio, V., and Patz, A. 2006. The effect of deforestation on the human-biting rate of Anopheles darlingi, the primary vector of Falciparum malaria in the Peruvian Amazon. Am. J. Trop. Med. Hyg. 74, 3-11.

Vong, S.,Coghlan,B.,Mardy, S.,Holl,D., Seng,H.,Ly, S.,Miller,M.J., P.Buchy, Froehlich, Y., Dufourcq, J.B., Uyeki, T.M., Lim, W., and Sok, T. 2006. Low frequency of poultry-to-human H5NI virus transmission, southern Cambodia, 2005. Emerg. Infect. Dis. 12, 1542-1547.

Wackernagel, M., Schulz, N.B., Deumling, D., Linares, A.C., Jenkins, M., Kapos, V., Monfreda, C., Loh, J., Myers, N., Norgaard, R., and Randers, J. 2002. Tracking the ecological overshoot of the human economy. Proc. Natl. Acad. Sci. USA. 99, 9266-9271.

Waddell, G.H., Teigland, M.B., and Sigel, M.M. 1963. A new influenza virus associated with equine viral respiratory disease. J. Am. Vet. Med. Assoc. 143, 587-590.

Wagenaar, J.A., Mevius, D.J., and Havelaar, A.H. 2006. Campylobacter in primary animal production and control strategies to reduce the burden of human campylobacteriosis. Rev. Sci. Tech. Off. Int. Epiz. 25, 581-594.

Wain-Hobson, S., and Meyerhans, A. 1999. On viral epidemics, zoonoses and memory. Trends in Microbiol. 7, 389-391.

Wales, C., Harvey, M., and Warde, A. 2006. Recuperating from BSE: The shifting UK institutional basis for trust in food. Appetite 47, 187-195.

Wall, P.G., Morgan, D., Lamden, K., Griffen, M., Threlfall, E.J., and Rowe, B. 1994. A case control study of infection with an epidemic strain of multiresistant Salmonella typhimurium DT104 in England and Wales. Commun. Dis. Rep. CDR Rev. 4, R130-R135.

Walsh, P.D., Abernethy, K.A., Bermejo, M., Beyers, R., P. De Wachter, M.E. Akou,Huijbregts,B.,Mambounga,D.I.,Toham,A.K.,Kilbourn,A.M.,Lahm, S.A., Latour, S., Maisels, F., Mbina, C., Mihindou, Y., Obiang, S.N., Effa, E.N., Starkey, M.P., Telfer, P., Thibault, M., Tutin, C.E., White, L.J., and Wilkie, D.S. 2003. Catastrophic ape decline in western equatorial Africa. Nature 422, 611-614.

Walther, B.A., and Ewald, P.W. 2004. Pathogen survival in the external environment and the evolution of virulence. Biol. Rev. Camb. Philos. Soc. 79, 849-869.

Waltner-Toews D., and Lang, T. 2000. A new conceptual base for food and agricultural policy: the emerging model of links between agriculture, food, health, environment and society. Glob. Change Hum. Health 1, 116-130.

Walton, J.R., and White, E.G., eds. 1981. Communicable Diseases Resulting from Storage Handling, Transport and Landspreading of Manure. Luxembourg: Office for Official Publications of the European Communities.

Wang, G., Zeng, Y.L., Liu, H.Y., and Xiong, Z.Y. 2007. An outbreak of Streptococcus suis in Chengdu, China. Int. J. Clin. Pract. 61, 1056-1057. 
Watkins, E.J., Brooksby, P., Schweiger, M.S., and Enright, S.M. 2001. Septicaemia in a pig-farm worker. Lancet 357, 38.

Webby, R.J., Swenson, S.L., Krauss, S.L., Gerrish, P.J., Goyal, S.M., and Webster, R.G. 2000. Evolution of swine H3N2 influenza viruses in the United States. J. Virol. 74, 8243-8251.

Webby, R.J., andWebster, R.G. 2001. Emergence of influenza A viruses. Philos. Trans. R. Soc. Lond. B. Biol. Sci. 356, 1817-1828.

Webby, R.J., Woolcock, P.R., Krauss, S.L., and Webster, R.G. 2002. Reassortment and interspecies transmission of North American H6N2 influenza viruses. Virology 295, 44-53.

Weber, D.J., and Rutala,W.A. 1999. Zoonotic infections. Occup. Med. State Art Rev. 14, 247-284.

Weber, T.P., and Stilianakis, N.I. 2007. Ecologic immunology of avian influenza (H5N1) in migratory birds. Emerg. Infect. Dis. Aug; [Epub ahead of print].

Webster, R.G. 2001. Virology: A molecular whodunit. Science 293, 1773-1775.

Webster, R.G. 2005. Session 1, Avian flu: Where do we stand? Conference on the Global Threat of Pandemic Influenza, Council on Foreign Relations, November 16.

Webster, R.G., Bean, W.J., Gorman, O.T., Chambers, T.M., and Kawaoka, Y. 1992. Evolution and ecology of influenza A viruses. Microbiol. Rev. 56, 152-179.

Webster, R.G., Geraci, J., Petursson, G., and Skirnisson, K. 1981. Conjunctivitis in human beings caused by influenza A virus of seals. N. Engl. J. Med. 30 4, 911.

Webster, R.G., Hulse-Post, D.J., Sturm-Ramirez, K.M., Guan, Y., Peiris, M., Smith, G., and Chen, H. 2007. Changing epidemiology and ecology of highly pathogenic avian H5N1 influenza viruses. Avian Dis. 51, 269-272.

Webster R.G., and Laver, W.G. 1971. Antigenic variation in influenza virus: Biology and chemistry. Prog. Med. Virol. 13, 271-338.

Webster, R.G., Laver,W.G., Air, G.M., and Schild, G.C. 1982. Molecular mechanisms of variation in influenza viruses. Nature 296, 115-121.

Webster, R.G., Peiris, M., Chen, H., and Guan, Y. 2006. H5N1 outbreaks and enzootic influenza. Emerg. Infect. Dis. 12, 3-8.

Webster, R.G., Shortridge, K.F., and Kawaoka, Y. 1997. Influenza: Interspecies transmission and emergence of new pandemics. FEMS Immunol. Med. Microbiol. 18, 275-279.

Webster, R.G., Wright, S.M., Castrucci, M.R., Bean, W.J., and Kawaoka, Y. 1993. Influenza: A model of an emerging virus disease. Intervirology 35, 16-25.

Webster, R.G., Yakhno, M., Hinshaw, V.S., Bean, W.J., and Murti, K.G. 1978. Intestinal influenza: Replication and characterization of influenza viruses in ducks. Virology 84, 268-278.

Weigler, B.J. 1992. Biology of B virus in macaque and human hosts: A review. Clin. Infect. Dis. 14, 555567.

Weinstein, M.R., Litt, M., Kertesz, D.A., Wyper, P., Rose, D., Coulter, M., A. McGeer, Facklam, R., Ostach, C., Willey, B.M., Borczyk, A., and Low, D.E. 1997. Invasive infections due to a fish pathogen, Streptococcus iniae. N. Engl. J. Med. 337, 589-594.

Weiss, R.A. 2001. Animal origins of human infectious disease, The Leeuwenhoek Lecture. Philos. Trans. R. Soc. Lond. B. Biol. Sci. 356, 957-977.

Weiss, R.A. 2003. Cross-species infections. Curr. Top. Microbiol. Immunol. 278, 47-71.

Weiss, R.A., and Wrangham, R.W. 1999. From Pan to pandemic. Nature 397, 385-386.

Welch, T.J., Fricke, W.F., McDermott, P.F., White, D.G., Rosso, M.L., Rasko, D.A., Mammel, M.K., Eppinger, M., Rosovitz, M.J., Wagner, D., Rahalison, L., Leclerc, J.E., Hinshaw, J.M., Lindler, L.E., Cebula, T.A., Carniel, E., and Ravel, J. 2007. Multiple antimicrobial resistance in plague: An emerging public health risk. PLOS ONE 2, e309.

Werner, J., and Gelderblom, H. 1979. Isolation of foamy virus from patients with de Quervain thyroiditis. Lancet 2, 258-259.

Wesley, I.V. 1999. Listeriosis in animals. In Listeria, listeriosis, and food safety, 2nd ed., eds. Ryser, E.T. and Marth, E.H., 39-73. New York, NY: Marcel Dekker Inc.

Westarp, M.E., Fuchs, D., Bartmann, P., Hoff-Jorgensen, R., Clausen, J., H. Wachter, and Kornhuber, H.H. 1993. Amyotrophic lateral sclerosis an enigmatic disease with B-cellular and anti-retroviral immune responses. Eur. J. Med. 2, 327-332.

Westbury, H.A., Turner, A.J., and Amon, C. 1981. Transmissibility of two avian influenza A viruses (H7N7) between chicks. Avian Pathol. 10, 481-487. 
Westbury, H.A., Turner, A.J., and Kovesdy, L. 1979. The pathogenicity of three Australian fowl plague viruses for chickens, turkeys and ducks. Vet. Microbiol. 4, 223-234.

White, P.C., and Benhin, J.K. 2004. Factors influencing the incidence and scale of bovine tuberculosis in cattle in southwest England. Prev. Vet. Med. 63, 1-7.

Whyte, P., Collins, J.D., McGill, K., Monahan, C., and O'Mahony, H. 2001. The effect of transportation stress on excretion rates of Campylobacter in market-age broilers. Poult. Sci. 80, 817-820.

Wilesmith, J.W., Ryan, J.B., and Atkinson, M.J. 1991. Bovine spongiform encephalopathy: Epidemiological studies on the origin. Vet. Rec. 128, 199-203.

Wilkie, D.S., Sidle, J.G., and Boundzanga, G.C. 1992. Mechanized logging, market hunting and a bank loan in Congo. Conserv. Biol. 6, 570-580.

Willenburg, K.S., Sentochnik, D.E., and Zadoks, R.N. 2006. Human Streptococcus suis meningitis in the United States. N. Engl. J. Med. 354, 1325.

William, J. 2003. The story of civet. Pharm. J. 271, 859-861.

Wilson, P.R. 2002. Advances in health and welfare of farmed deer in New Zealand. NZ Vet. J. 50, 105109.

Wilson, T.M., Logan-Henfrey, L.,Weller, R., and Kellman, B. 2000. Agroterrorism, biological crimes, and biological warfare targeting animal agriculture. In Emerging diseases of animals, ed. Brown, C., 23-57. Washington, D.C.: ASM Press.

Wilson, W.O. 1966. Poultry production. Sci. Am. 215, 56-64.

Winternitz, M.C., Wason, I.M., and McNamara, F.P. 1920. The Pathology of Influenza. New Haven, CT: Yale University Press.

Witter, R.L. 1998. Control strategies for Marek's disease: A perspective for the future. Poult. Sci. 77, 1197-1203.

Wobeser, G.A. 1997. Diseases ofWildWaterfowl. New York, NY: Plenum Press.

Wolfe, N.D., Dunavan, C.P., and Diamond, J. 2007. Origins of major human infectious diseases. Nature 447, 279-283.

Wolfe, N.D., Eitel, M.N., Gockowski, J., Muchaal, P.K., Nolte, C., Prosser, A.T., Torimiro, J.N., Weise, S.F., and Burke, D.S. 2000. Deforestation, hunting and the ecology of microbial emergence. Glob. Change Hum. Health 1, 10-25.

Wolfe, N.D., Heneine, W., Carr, J.K., Garcia, A.D., Shanmugam, V., Tamoufe, U., Torimiro, J.N., Prosser, A.T., Lebreton, M., E. Mpoudi-Ngole, McCutchan, F.E., Birx, D.L., Folks, T.M., Burke, D.S., and Switzer, W.M. 2005. Emergence of unique primate T-lymphotropic viruses among central African bushmeat hunters. Proc. Natl. Acad. Sci. USA 10 2, 7994-7999.

Wolfe,N.D., Switzer,W.M., Carr, J.K., Bhullar,V.B., Shanmugam,V., Tamoufe, U., Prosser, A.T., Torimiro, J.N., Wright, A., Mpoudi-Ngole, E., McCutchan, F.E., Birx, D.L., Folks, T.M., Burke, D.S., and Heneine, W. 2004. Naturally acquired simian retrovirus infections in central African hunters. Lancet 363, 932-937.

Woo, P.C., Lau, S.K., and Yuen, K.Y. 2006. Infectious diseases emerging from Chinese wet-markets: Zoonotic origins of severe respiratory viral infections. Curr. Opin. Infect. Dis. 19, 401-417.

Woodford, M.H., and Rossiter, P.B. 1993. Disease risks associated with wildlife translocation projects. Rev. Sci. Tech. Off. Int. Epiz. 12, 115-135.

Woolhouse, M.E. 2002. Population biology of emerging and re-emerging pathogens. TIM 10, S3-S7.

Woolhouse, M.E., and Gowtage-Sequeria, S. 2005. Host range and emerging and reemerging pathogens. Emerg. Infect. Dis. 11, 1842-1847.

World Health Organization (WHO) Expert Committee. 1980a. A revision of the system of nomenclature for influenza viruses. Bull. World Health Organ. 58, 585-591.

World Health Organization (WHO). 1980b. Declaration of global eradication of smallpox. Wkly. Epidemiol. Rec. 55, 145-152.

World Health Organization (WHO). 1996. Outbreak of Ebola haemorrhagic fever in Gabon officially declared over. Wkly. Epidemiol. Rec. 71, 125-126.

World Health Organization (WHO), Department of Communicable Disease Surveillance. 1999. Future trends in veterinary public health. Wkly. Epidemiol. Rec. 74, 154-156.

World Health Organization (WHO). 2003. Outbreak(s) of Ebola haemorrhagic fever, October 2001-July 2002. Wkly. Epidemiol. Rec. 78, 223-228.

World Health Organization (WHO). 2004. Summary table of SARS cases by country, November 1, 2002July 31, 2003. 
World Health Organization (WHO). 2005. Streptococcus suis fact sheet.

WorldHealthOrganization (WHO). 2006a.Global tuberculosis control: Surveillance, planning, financing.

World Health Organization (WHO) Working Group. 2006b. Influenza research at the human and animal interface. Geneva, Switzerland, September 21-22.

World Health Organization (WHO). 2006c. Review of latest available evidence on risks to human health through potential transmission of avian influenza (H5N1) through water and sewage.

World Health Organization (WHO) Western Pacific Regional Office. 2007. Avian influenza update 100. June 19.

World Health Organization, Food and Agriculture Organization of the United Nations, andWorld Organization for Animal Health (WHO/FAO/OIE). 2003. Expert workshop on non-human antimicrobial usage and antimicrobial resistance, December 1-5, Geneva.

World Health Organization, Food and Agriculture Organization of the United Nations, andWorld Organization for Animal Health (WHO/FAO/OIE). 2004. Report of the WHO/FAO/OIE joint consultation on emerging zoonotic diseases. <whqlibdoc.who.int/hq/2004/WHO CDS CPE ZFK 2004.9.pdf>.

World Health Organization and Office International des Epizooties (WHO \& OIE). 1999. WHO consultation on public health and animal transmissible spongiform encephalopathies: Epidemiology, risk and research requirements, December 1-31.

World Organization for Animal Health (OIE). 2007. OIE update on avian influenza in animals (type H5). August 6.

Worm, H.C., van der Poel, W.H., and Brandstatter, G. 2002. Hepatitis E: An overview. Microb. Infect. 4, 657-666.

Wuethrich, B. 2003a. Infectious disease: An avian flu jumps to people. Science 299, 1504.

Wuethrich, B. 2003b. Infectious disease: Chasing the fickle swine flu. Science 299, 1502-1505.

Xin, X.,Wei-Ming, T., and Zhang-Yue, Z. 2001. Changing patterns of feedgrain production and marketing in China. Australasian Agribusiness Perspectives Paper 47.

Xu, K.M., Smith, G.J., Bahl, J., Duan, L., Tai, H., Vijaykrishna, D., Wang, J., Zhang, J.X., Li, K.S., Fan, X.H., Webster, R.G., Chen, H., Peiris, J.S., and Guan,Y. 2007. The genesis and evolution of H9N2 influenza viruses in poultry from southern China, 2000 to 2005. J. Virol. Jul 25; [Epub ahead of print].

Xu, R.H., He, J.F., Evans, M.R., Peng, G.W., Field, H.E., Yu, D.W., Lee, C.K., Luo, H.M., Lin,W.S., Lin, P., Li, L.H., Liang,W.J., Lin, J.Y., and Schnur, A. 2004. Epidemiologic clues to SARS origin in China. Emerg. Infect. Dis. 10, 1030-1037.

Yang, Y., Halloran, M.E., Sugimoto, J., and Longini, I.M., Jr. 2007. Detecting human-to-human transmission of avian influenza A (H5N1). Emerg. Infect. Dis. Sep; [Epub ahead of print].

Yi, C.E., Ba, L., Zhang, L., Ho, D.D., and Chen, Z. 2005. Single amino acid substitutions in the severe acute respiratory syndrome coronavirus spike glycoprotein determine viral entry and immunogenicity of a major neutralizing domain. J. Virol. 79, 11638-11646.

Yoo, D.,Willson, P., Pei, Y., Hayes, M.A., Deckert, A., Dewey, C.E., Friendship, R.M., Yoon, Y., Gottschalk, M., Yason, C., and Giulivi, A. 2001. Prevalence of hepatitis E virus antibodies in Canadian swine herds and identification of a novel variant of swine hepatitis E virus. Clin. Diagn. Lab. Immunol. 8, 1213-1219.

Yu, H., Jing, H., Chen, Z., Zheng, H., Zhu, X., Wang, H., Wang, S., Liu, L., Zu, R., Luo, L., Xiang, N., Liu, H., Liu, X., Shu, Y., Lee, S.S., Chuang, S.K., Wang, Y., Xu, J., and Yang, W. 2006. Human Streptococcus suis outbreak, Sichuan, China. Emerg. Infect. Dis. 12, 914-920.

Yunis, R., Ben-David, A., Heller, E.D., and Cahaner, A. 2000. Immunocompetence and viability under commercial conditions of broiler groups differing in growth rates and in antibody response to Escherichia coli vaccine. Poult. Sci. 79, 810-816.

Zanella, A. 2002. Avian influenza attributable to Serovar H7N1 in light layers in Italy. Avian Dis. 47, 11771180.

Zavala, G. 1998. An overview of myeloid leukosis in meat-type chickens. Technical News, Special Technical Bulletin, January: S1-S4.

Zepeda, C., and Salman, M.D. 2007. Assessing the probability of the presence of low pathogenicity avian influenza virus in exported chicken meat. Avian Dis. 51, 344-351.

Zhang, G., Shoham, D., Gilichinsky, D., Davydov, S., Castello, J.D., and S.O. Rogers. 2006. Evidence of influenza a virus RNA in Siberian lake ice. J. Virol. 80, 12229-12235. 
Zhao, G.P. 2007. SARS molecular epidemiology: A Chinese fairy tale of controlling an emerging zoonotic disease in the genomics era. Philos. Trans. R. Soc. Lond. B. Biol. Sci. 362, 1063-1081.

Zhao, J., and Aoki, T. 1992. Nucleotide sequence analysis of the class $G$ tetracycline resistance determinant from Vibrio anguillarum. Microbiol. Immunol. 36, 1051-1060.

Zhichun Yan, J., Morrison, B., Meyer, T., and Deen, J. 2003. Inside China. Pig Progress 19, 9-14.

Zhou, N.N., Senne, D.A., Landgraf, J.S., Swenson, S.L., Erickson, G., Rossow, K., Liu, L., Yoon, K., Krauss, S., and Webster, R.G. 1999. Genetic reassortment of avian, swine, and human influenza A viruses in American pigs. J. Virol. 73, 8851-8856.

Zlotkin, A., Hershko, H., and Eldar, A. 1998. Possible transmission of Streptococcus iniae from wild fish to cultured marine fish. Appl. Environ. Microbiol. 64, 4065-4067.

Zobeley, E., Flechsig, E., Cozzio, A., Enari, M., and Weissmann, C. 1999. Infectivity of scrapie prions bound to a stainless steel surface. Mol. Med. 5, 240-243. 